\author{
Universidade de São Paulo \\ Instituto de Física
}

\title{
Condensados de Bose-Einstein com interação spin-órbita
}

\author{
Alex Valerio Andriati
}

Orientador: Prof. Dr. Arnaldo Gammal

Dissertação de mestrado apresentada ao Instituto de Física da Universidade de São Paulo, como requisito parcial para a obtenção do título de Mestre em Ciências. Área de concentração: Física Atômica.

Banca Examinadora:

Prof. Dr. Arnaldo Gammal - Orientador (Instituto de Física - USP)

Prof. Dr. Francisco Ednilson Alves dos Santos - UFSCAR (via videoconferência)

Prof. Dr. Lauro Tomio - IFT UNESP

São Paulo 


\section{FICHA CATALOGRÁFICA \\ Preparada pelo Serviço de Biblioteca e Informação do Instituto de Física da Universidade de São Paulo}

Andriati, Alex Valerio

Condensados de Bose-Einstein com interação spin-órbita.

São Paulo, 2018.

Dissertação (Mestrado) - Universidade de São Paulo. Instituto de Física. Depto. de Física Experimental

Orientador: Prof. Dr. Arnaldo Gammal

Área de Concentração: Física Atômica e Molecular

Unitermos: 1. Condensado de Bose-Einstein; 2. Interação spin-órbita; 3. Interação atômica atrativa. 
Ao meu filho, com carinho, por toda felicidade e amadurecimento durante o período deste trabalho. 


\section{Agradecimentos}

Agradeço de forma geral a todos amigos e familiares que estiveram presentes e, que prestaram apoio, motivação e incentivo não só em momentos felizes, mas também nos mais difíceis.

Especificamente ao Prof. Dr. Arnaldo Gammal, por toda paciência e didática em sua orientação, por tudo que veio a acrescentar em conhecimento técnico-científico e, pela dedicação que atendeu a toda solicitação.

Aos professores Antônio Fernando Ribeiro de Toledo Piza e Emerson José Veloso de Passos pelas frutíferas discussões, nas reuniões semanais do "Journal Club", que veio a enriquecer este trabalho.

Ao professor Marcos Godoy, pelo primeiro contato com a física e, que sem sua motivação para prosseguir nos estudos em nível superior, este trabalho nunca sequer teria acontecido.

Aos colaboradores do Instituto de Física, especialmente da Comissão de Pós-Graduação (CPG), ao Centro de Computação do Instituto de Física e as secretarias, pela atenção e eficiência nos serviços prestados.

Por fim e mais essencial, ao Conselho Nacional de Desenvolvimento Científico e Tecnológico, CNPQ, que sem o auxílio financeiro, esta dissertação não seria possível. 


\section{Resumo}

Nesta dissertação são estudados Condensados de Bose-Einstein de átomos com pseudo-spin $1 / 2$ cuja dinâmica orbital está acoplada a estes dois níveis de energia internos. A geração de tal sistema é possível induzindo transições entre os subníveis $m_{f}=-1$ e $m_{f}=0$ do estado hiperfino atômico $f=1$ usando um arranjo de lasers, os quais também introduzem junto uma dependência espacial dada por suas fases, as quais estão relacionadas a posição do átomo no campo, levando assim à interação acoplando spin e órbita. É considerado então um sistema unidimensional efetivo na mesma direção do acoplamento dos lasers, onde são estudado diferentes observáveis do estado fundamental, para uma varredura dos parâmetros presentes na equação, dando origem a três fases diferenciadas pela distribuição do momento. Foram determinadas estas fases do estado fundamental para interação atrativa, sendo elas modulada(striped), onda plana e de momento nulo, mostrando a localização onde cada uma ocorre no domínio de parâmetros da equação, através de diagramas de fase. São também mostrados, separadamente, observáveis relevantes como momento e desbalanço entre os estados internos nestas transições, os quais apresentaram variações bruscas, ditando valores críticos nos parâmetros, onde ocorrem. Posteriormente é estudado a dinâmica através de soluções do tipo sóliton, as quais não se propagam linearmente e são ditadas por oscilações do centro de massa e das populações, explorando diferentes situações iniciais.

Palavras-Chave: Condensado de Bose-Einstein, interação spin-órbita, interação atômica atrativa. 


\begin{abstract}
In the present dissertation it has been studied Bose-Einstein Condensation of atoms with $1 / 2$ pseudospin whose the orbital dynamics is coupled to these two internal energy levels. The generation of such a system is done by inducing transitions between the sub-levels $m_{f}=-1$ and $m_{f}=0$ from the hyperfine atomic state $f=1$ using an arrangement of lasers, that also introduce a spacial dependence due to their phases, that changes accordingly the atom's position in the light field, conducting in this way to a interaction that couples orbital motion with spin. It is then considered an effective one dimensional system in the same direction of the laser coupling, where it has been studied different ground state observables, making a sweeping in the equation parameters, showing three typical phases based on momentum distribution. So far, it was determined these phases for attractive interactions, named striped, plane wave and zero momentum, determining as well the location where each one occurs in the equation's parameters through a phase diagram. It is also reported, separately, a few relevant observables as individual momentum of each population and the unbalance between the internal spin states, in the transition among these phases, whose the values present abrupt variations, dictating critical values for the parameters, where it occurs. Lately is presented a dynamical study with soliton like solutions, that do not linearly propagate and instead, shows a center of mass and unbalance oscillation, probing different initial conditions.
\end{abstract}

keywords: Bose-Einstein condensate, spin-orbit interaction, attractive atomic interactions. 


\section{Sumário}

1 Introdução $\quad 7$

1.1 Por que estudar gases a baixas Temperaturas? . . . . . . . . . . . . 7

1.2 Rudimentos da Condensação de Bose-Einstein e estrutura hiperfina atômica . . 10

1.3 Pretensão deste trabalho . . . . . . . . . . . . . . . . . . . 13

2 acoplamento spin-órbita $\quad 14$

2.1 Breve discussão de Segunda Quantização . . . . . . . . . . . . . . . . . . 14

2.1 .1 Bósons e Férmions . . . . . . . . . . . . . . . . . . 16

2.1.2 Operadores de Campo e Densidade com Spin . . . . . . . . . . . . 18

2.1.3 Representação de Operadores . . . . . . . . . . . . . . . . . . 19

2.1.4 Dinâmica de operadores . . . . . . . . . . . . . 21

2.2 Interação Efetiva a baixas temperaturas . . . . . . . . . . . . 22

2.2.1 Aproximação de Born e um critério de validade . . . . . . . . . . . 23

2.2.2 "Scattering Lenght" . . . . . . . . . . . . . . . . . . . . 24

2.3 Descrição de Campo Médio . . . . . . . . . . . . . . . . . . . 25

2.3.1 Estados Estacionários . . . . . . . . . . . . . . . . 29

2.3 .2 Ação do Campo . . . . . . . . . . . . . . . . . . . . . . . . . . 29

2.3.3 Soluções do tipo soliton . . . . . . . . . . . . . . 30

2.4 Condensado na presença de Lasers . . . . . . . . . . . . . . . . . 31

2.4.1 Elementos de interação Átomo-Luz sob aproximação de Dipolo . . . . 31

2.4 .2 Transições Raman . . . . . . . . . . . . . . . . . . . . 33

2.4.3 Escalas de energia e unidades . . . . . . . . . . . . 38

3 Métodos Numéricos $\quad 42$

3.1 Algoritmos de Evolução Temporal . . . . . . . . . . . . . . . . . . . . . 42 
3.1.1 Diferenças finitas, método Crank-Nicolson . . . . . . . . . . . . . 43

3.1 .2 Métodos Espectrais . . . . . . . . . . . . . . . 44

3.1.3 Procedimento de separação de operadores . . . . . . . . . . . . . 45

3.2 Algoritmos para soluções Estacionárias . . . . . . . . . . . . . . . . 46

3.2.1 Propagação em Tempo imaginário - Relaxação . . . . . . . . . . . . . 46

3.2.2 Estados Estacionários genéricos . . . . . . . . . . . . . 47

4 Resultados e Estudos Dirigidos

4.1 Autoestados sem interação . . . . . . . . . . . . . . . . . . 56

4.2 Estados Estacionários com Interação atrativa . . . . . . . . . . . . . . . . 59

4.3 Estudo de Fases baseado em Cálculo Variacional _ . . . . . . . . . . . . . 62

4.3.1 Diagramas com $k_{L}$ fixado . . . . . . . . . . . . 65

4.3.2 Efeito de $\delta$ sobre o Estado Fundamental . . . . . . . . . . . . . . 70

4.4 Dinâmica de Sólitons . . . . . . . . . . . . . . . . . . . . 73

5 Conclusões e Perspectivas $\quad 78$

A Cálculo Variacional para Dinâmica de Sólitons $\quad 81$

B Cálculo Variacional para o Estado Fundamental $\quad 85$ 


\section{Capítulo 1}

\section{Introdução}

\subsection{Por que estudar gases a baixas Temperaturas?}

Uma parcela expressiva de pesquisa tem sido feita, mais intensamente no último século, em temas que se correlacionam a temperaturas próximas a zero. $\mathrm{O}$ interesse nesse tema tem uma de suas raízes em entender e testar alguns dos postulados mais básicos da Física contemporânea, o principio de simetrização para Férmions e Bósons através da dinâmica de um conjunto de átomos.

As primeiras pesquisas relevantes datam mesmo antes da Teoria Quântica alcançar a total maturidade em meados de 1927-1930, na observação de fenômenos sem prescrição teórica na transição do Hélio para fase líquida, nos primeiros anos do mesmo século, mais tarde relacionado com a superfluidez [1].

Embora princípios termodinâmicos, que até então se mostram válidos, asseguram a existência de um limite mínimo para temperatura, denominado "zero absoluto", tal limite vem sendo progressivamente alcançado. Esse constante esforço experimental resultou na observação da Condensação de Bose-Einstein [2] em 1995, datando sua primeira previsão de 1924-1925 por Satyendra Nath Bose e Albert Einstein em [3] e [4], respectivamente.

De volta ao princípio de simetrização, um cálculo introdutório que aqui será feito, resulta numa fácil percepção do que difere com os postulados de simetria e, fornece significa quantitativo a "baixas temperaturas".

Seja $\mathcal{P}\left(i_{k}\right) \operatorname{com} k, i_{k} \in \mathbb{N} ; i, k<n$, o operador de permuta, que troca a $i$-ésima partícula com a $k$-ésima, por exemplo, se temos um estado $\left|k k^{\prime}\right\rangle \doteq|k\rangle_{1}\left|k^{\prime}\right\rangle_{2}$ onde a partícula enumerada 1 tem momento $\hbar k$ e a 2 possui $\hbar k^{\prime}$, a ação de $\mathcal{P}\left(2_{1}\right)$ leva ao estado em que a 1 terá momento 
$\hbar k^{\prime}$ e 2 possuirá $\hbar k$, i.e, $\mathcal{P}\left(1_{2}\right)\left|k k^{\prime}\right\rangle=\left|k^{\prime} k\right\rangle$. Segue que

1. $\mathcal{P}\left(i_{k}\right)^{2}=\mathcal{P}\left(i_{i}\right)=\mathbb{1}$;

2. $\mathcal{P}\left(i_{k}\right)^{\dagger}=\mathcal{P}\left(i_{k}\right)$;

3. Se $[\mathcal{O}, \mathcal{P}]=0$, então $\mathcal{O}$ não depende de ordenamento.

Tal operador pode ainda ser generalizado para permutas quaisquer sobre um conjunto $\left\{i_{1}, \ldots, i_{n}\right\}$ com $\mathcal{P}\left(i_{1}, \ldots, i_{n}\right)$ indicando a troca da partícula $1 \mathrm{com}$ a de número $i_{1}$, e analogamente para o restante. O número de permutas é então definido pela quantidade de $i_{l}$ tais que $l \neq i$ que será denotado por $\mathcal{N}_{p}$.

Os postulados de simetrização exigem que para qualquer estado genérico de $n$ partículas tenhamos este como autoestado de qualquer permuta, com

$$
\mathcal{P}\left(i_{1}, \ldots, i_{n}\right)\left|\Psi^{(n)}\right\rangle=( \pm 1)^{\mathcal{N}_{p}}\left|\Psi^{(n)}\right\rangle=\left\{\begin{array}{c}
(+) \text { Bosons } \\
(-) \text { Fermions }
\end{array}\right\}
$$

A Hamiltoniana de $n$ partículas não interagentes, mostra o caso de um operador separável, i.e, onde seus autoestados podem ser construídos como o produto dos estados individuais na forma

$$
\mathcal{H}=\sum_{l=1}^{n} \frac{p^{2}}{2 m} ; \quad \mathcal{H}\left|\mathbf{k}_{\mathbf{1}} \ldots \mathbf{k}_{\mathbf{n}}\right\rangle=\left(\sum_{l=1}^{n} \frac{\hbar^{2} k_{l}^{2}}{2 m}\right)\left|\mathbf{k}_{\mathbf{1}} \ldots \mathbf{k}_{\mathbf{n}}\right\rangle .
$$

Passa-se então a exigir que tais auto estados estejam (anti)simetrizados com relação a (Fermions)Bósons, fazendo a mudança

$$
\left|\mathbf{k}_{1} \ldots \mathbf{k}_{n}\right\rangle \mapsto\left|\left\{\mathbf{k}_{1}, \ldots \mathbf{k}_{n}\right\}\right\rangle=\frac{1}{\sqrt{n_{\mathbf{k}_{1}} ! \ldots n_{\mathbf{k}_{n}} !}} \frac{1}{\sqrt{n !}} \sum_{\mathcal{P}}( \pm 1)^{\mathcal{N}_{p}} \mathcal{P}\left(\left\{i_{k}\right\}\right)\left|\mathbf{k}_{1} \ldots \mathbf{k}_{n}\right\rangle
$$

onde a diferente notação incluindo \{\} atenta para o fato de que são autoestados simultâneos de $\mathcal{P}$ e $\mathcal{H}$ e podem ser escritos como uma combinação dos apresentados em (1.2).

Para ilustrar então a consequência desse postulado de simetria, podemos calcular a densidade de probabilidade de encontrar partículas nas posições $\mathbf{r}_{1}, \ldots, \mathbf{r}_{n}$. Isso pode ser feito por intermédio da matriz densidade no Ensemble Canônico, com as propriedades das permutações, iniciando com

$$
\Phi\left(\mathbf{r}_{1}, \ldots, \mathbf{r}_{n}\right) \doteq\left\langle\left\{\mathbf{r}_{1} \ldots \mathbf{r}_{n}\right\}|\rho|\left\{\mathbf{r}_{1} \ldots \mathbf{r}_{n}\right\}\right\rangle, \quad \rho \doteq \mathrm{e}^{-\beta \mathcal{H}}
$$


temos,

$$
\begin{aligned}
\Phi\left(\mathbf{r}_{1}, \ldots, \mathbf{r}_{n}\right) & =\left\langle\left\{\mathbf{r}_{1} \ldots \mathbf{r}_{n}\right\}\left|\rho \frac{1}{\sqrt{n !}} \sum_{\mathcal{P}}( \pm 1)^{\mathcal{N}_{p}} \mathcal{P}\left(\left\{i_{k}\right\}\right)\right| \mathbf{r}_{1} \ldots \mathbf{r}_{n}\right\rangle \\
& =\frac{1}{\sqrt{n !}} \sum_{\mathcal{P}}\left\langle\left\{\mathbf{r}_{1} \ldots \mathbf{r}_{n}\right\}\left|\mathcal{P}\left(\left\{i_{k}\right\}\right)( \pm 1)^{\mathcal{N}_{p}} \rho\right| \mathbf{r}_{1} \ldots \mathbf{r}_{n}\right\rangle \\
& =\frac{1}{\sqrt{n !}} \sum_{\mathcal{P}}\left\langle\left\{\mathbf{r}_{1} \ldots \mathbf{r}_{n}\right\}|\rho| \mathbf{r}_{1} \ldots \mathbf{r}_{n}\right\rangle \\
& =\sqrt{n !}\left\langle\left\{\mathbf{r}_{1} \ldots \mathbf{r}_{n}\right\}|\rho| \mathbf{r}_{1} \ldots \mathbf{r}_{n}\right\rangle \\
& =\sqrt{n !} \sum_{\mathbf{k}_{1} \ldots \mathbf{k}_{n}}\left\langle\left\{\mathbf{r}_{1} \ldots \mathbf{r}_{n}\right\} \mid \mathbf{k}_{1} \ldots \mathbf{k}_{n}\right\rangle \frac{\mathrm{e}^{-\beta E\left(\mathbf{k}_{1}, \ldots, \mathbf{k}_{n}\right)}}{Z}\left\langle\mathbf{k}_{1} \ldots \mathbf{k}_{n} \mid \mathbf{r}_{1} \ldots \mathbf{r}_{n}\right\rangle \\
& =\frac{1}{Z} \sum_{\mathcal{P}}( \pm 1)^{\mathcal{N}_{p}} \sum_{\mathbf{k}_{1} \ldots \mathbf{k}_{n}} \prod_{l=1}^{n} \exp \left(-\beta \frac{\hbar^{2} k_{l}^{2}}{2 m}\right) \phi_{\mathbf{k}_{l}}^{*}\left(\mathbf{r}_{\mathcal{P}(l)}\right) \phi_{\mathbf{k}_{l}}\left(\mathbf{r}_{l}\right) .
\end{aligned}
$$

onde foi expresso a matriz densidade na base referente a (1.2). As funções de partícula, $\phi_{\mathbf{k}_{j}}$, livre são tomadas como periódicas em um volume $V$. Invertendo soma e produto e passando a integração, temos

$$
\sum_{\mathbf{k}_{1} \ldots \mathbf{k}_{n}} \prod_{l=1}^{n} \equiv \prod_{l=1}^{n} \sum_{\mathbf{k}_{l}} \longmapsto \prod_{l=1}^{n} \frac{V}{(2 \pi)^{3}} \int \mathrm{d}^{3} \mathbf{k}_{l},
$$

resultando numa transformada de Fourier de uma Gaussiana, pelo fator exponencial das funções $\phi$ de partícula livre, finalmente conduzindo a

$$
\begin{aligned}
\Phi\left(\mathbf{r}_{1}, \ldots, \mathbf{r}_{N}\right) & =\frac{1}{Z(T, V, N)\left(\lambda_{T}\right)^{3 N}} \sum_{\mathcal{P}}( \pm 1)^{\mathcal{N}_{p}} \prod_{l=1}^{N} \exp \left[-\frac{\pi}{\lambda_{T}^{2}}\left(\mathbf{r}_{l}-\mathbf{r}_{\mathcal{P}(l)}\right)^{2}\right] \\
\lambda_{T} & \doteq h / \sqrt{2 m \pi k_{B} T}
\end{aligned}
$$

$\operatorname{com} \lambda_{T}$ dito comprimento de onda térmico. Para $N=2$ encontra-se

$$
\Phi\left(\mathbf{r}_{1}, \mathbf{r}_{2}\right)=\frac{1}{Z(T, V, 2)\left(\lambda_{T}\right)^{6}}\left\{1 \pm \exp \left[-\frac{2 \pi}{\lambda_{T}^{2}}\left(\mathbf{r}_{1}-\mathbf{r}_{2}\right)^{2}\right]\right\}
$$

resultado particular mostrado em [5, exemplo 11.2], onde a função de partição também é detalhada e, vem a ser

$$
Z(T, V, 2)=\frac{V^{2}}{2 \lambda_{T}^{6}}\left(1 \pm \frac{\lambda_{T}^{3}}{\sqrt{8} V}\right) .
$$

Portanto dependendo do sinal, ou seja, Férmions ou Bósons, a densidade de Probabilidade das partículas serem encontradas mais próximas é menor ou maior, respectivamente. Contudo este efeito se pronuncia espacialmente a medida que $\lambda_{T}$ é maior possível, que se relaciona com 
desvio padrão na Gaussiana (1.6) e, isso equivale a $T \rightarrow 0$.

Portanto estudar sistemas a baixas temperaturas mostra não somente o uso da descrição Quântica, mas também uma plataforma onde efeitos se simetrização se pronunciam, cuja a presente dissertação irá abordar como se comportam Bósons, no fenômeno da Condensação de Bose-Einstein. Este efeito é automaticamente contabilizado no formalismo que se nomeia “segunda quantização", o qual será adotado e explicitado a frente.

\subsection{Rudimentos da Condensação de Bose-Einstein e estru- tura hiperfina atômica}

Segue diretamente do Ensemble Macro-Canônico, o cálculo do valor médio do número de ocupação para um gás de Bosons como sendo

$$
\left\langle n_{k}\right\rangle=\left(\frac{\mathrm{e}^{\beta \epsilon_{k}}}{z}-1\right)^{-1}=\frac{1}{\mathrm{e}^{\beta\left(\epsilon_{k}-\mu\right)}-1},
$$

onde $z \doteq \mathrm{e}^{\beta \mu}$ é conhecida como fugacidade e, este resultado pode ser amplamente encontrado, por exemplo em [5, 6].

Para que os valores acima sejam positivos definidos, é necessário que $\mu<\epsilon_{k}, \forall k$, sempre que $T>0$. Porém a medida que $T \rightarrow 0 \Rightarrow \beta \rightarrow \infty$, naturalmente $\left\langle n_{k}\right\rangle \rightarrow 0$. Portanto para que eventualmente permaneçam partículas no sistema a inequação $\mu<\epsilon_{o}$ deve se aproximar de uma igualdade. Por assim dizer,

$$
T \rightarrow 0 \Rightarrow \mu \rightarrow \epsilon_{o} ; \quad\left\langle n_{o}(T=0)\right\rangle \equiv N
$$

Caracterizando a ocupação majoritária do estado fundamental de partícula individual. Estimativas são possíveis para a fração de ocupação do estado fundamental para uma temperatura bem próxima de zero, mais especificamente abaixo de um ponto crítico, dependendo da Hamiltoniana do sistema. No gás livre, por exemplo, temos a fração ocupando o estado fundamenta $N_{o}$ dada por

$$
\frac{N_{o}}{N}=1-\left(\frac{T}{T_{c}}\right)^{3 / 2}, \quad k_{B} T_{c}=\frac{2 \pi \hbar^{2}}{m}\left(\frac{N}{\zeta(3 / 2) V}\right)^{2 / 3}
$$

onde $\zeta$ indica a função Zeta de Riemann [5, cap. 13]. 
O caso dos átomos num potencial harmônico (sendo comum a referência "Armadilha"), também pode ser tratado analiticamente, conduzindo no entanto a um resultado levemente diferente do anterior, com a fração agora dada por

$$
\frac{N_{o}^{\prime}}{N}=1-\left(\frac{T}{T_{c}^{\prime}}\right)^{3}, \quad k_{B} T_{c}^{\prime}=\hbar^{2}\left(\frac{N \omega_{x} \omega_{y} \omega_{z}}{\zeta(3)}\right)^{1 / 3}
$$

mostrado em [7, cap. 10], de especial interesse experimental, devido a arranjos de campos magnéticos e interação de átomos com luz que podem gerar tal potencial, onde neste caso, $\omega_{i}$ vem a depender da intensidade dos campos em questão [8, 7].

Apesar dos resultados acima demonstratem boa acurácia para um sistema dito diluto, onde as colisões tem efeito minoritário, é possível desenvolver um critério mais genérico para a condensação de Bose-Einstein incluindo interações, como explicitado em [9]. Este critério, baseia-se na matriz densidade reduzida(como encontrado neste última referência), obtida pelo traço no subespaço gerado por $N-1$ consituintes, cujo o autovalor, se representada no número de ocupação, deve caracterizar esta ocupação macroscópica do estado fundamental, muito embora qualquer pretensão analítica é mais engenhosa de se obter.

Apesar das considerações anteriores, os níveis de energia não residem apenas na translação, ou seja, o termo cinético, pois há também a estrutura interna dos constituintes. Isto advém da separação na hamiltoniana nas coordenadas relativas ao centro de massa: $E_{a t}=E^{C M}+\mathcal{E}$.

Posto de outra forma, ao se tratar da condensação de átomos, podemos explorar a estrutura dos níveis de energia internos, onde na presente tese será abordado o momento angular atômico $(f)$, este constituído da soma da parte orbital $(j)$ com a nuclear $(i)$.

Momento angular intrínseco("spin”) de partículas carregadas induzem a interações por carregarem momento magnético, muito embora sejam energeticamente menos expressivos que o potencial de Coulomb e seja suficiente uma abordagem perturbativa para estudar seus efeitos. Por menos expressiva, quantitativamente significa que a razão da correção em primeira ordem pela energia não perturbada é proporcional a constante de estrutura fina $\alpha \approx 1 / 137$ multiplicada pela razão $m_{e} / m_{p}$, detalhado em [8] cap. 3 e baseado em [10] seções 113, 121.

No contexto da condensação de átomos bosônicos ("spin" atômico inteiro), o menor nível de energia interno é fixado pelo estado fundamental segundo a disposição dos elétrons, decorrente da interação coulombiana com o núcleo, que em primeira aproximação ${ }^{1}$, se distribuem segundo os níveis de energia do átomo de hidrogênio, respeitando o princípio de exclusão de Pauli.

\footnotetext{
${ }^{1}$ Genericamente desconsiderando a interação entre elétrons
} 
Como mencionado acima, a distribuição orbital dos elétrons para átomos alcalinos deve portanto ter $j=s=1 / 2$ uma vez que a única contribuição vem do spin do elétron na camada mais energética, pois o resto dos orbitais não contribuem ${ }^{2}$, já que estão em pares nos níveis abaixo e devem estar em estado de singleto (férmions $\rightarrow$ antisimetria). Valores de $i$ dependem dos diferentes isótopos e caracterizarão se as espécies serão Bósons ou Férmions.

Os momentos angulares citados anteriormente se relacionam, em seus operadores, por $\hat{F}=$ $\hat{I}+\hat{J}$. A interação proveniente dos spins nuclear e eletrônico devido a seus momentos magnéticos é nomeada interação hiperfina. Claramente a interação hiperfina depende do operador posição, no entanto, usando teoria perturbativa podemos escrever uma Hamiltoniana efetiva

$$
\mathcal{H}_{h f}=A \mathbf{I} \cdot \mathbf{J}=\frac{A}{2}[f(f+1)-i(i+1)-3 / 4]
$$

Com separação(split) de energia entre os níveis com $f=i \pm 1 / 2$, ainda dependendo do átomo que influencia $i$ e $A$, com $\Delta E_{h f}=(i+1 / 2) A$. A tabela 1 de [11] tem alguns dos principais átomos e isótopos transcritos abaixo.

\begin{tabular}{|c|c|c|c|c|}
\hline Átomo & $j$ & $i$ & $f$ & $\Delta E_{h f} / h(\mathrm{MHz})$ \\
\hline${ }^{1} \mathrm{H}$ & $1 / 2$ & $1 / 2$ & 0,1 & 1420 \\
\hline${ }^{7} \mathrm{Li}$ & $1 / 2$ & $3 / 2$ & 1,2 & 804 \\
\hline${ }^{23} \mathrm{Na}$ & $1 / 2$ & $3 / 2$ & 1,2 & 1772 \\
\hline${ }^{39} \mathrm{~K}$ & $1 / 2$ & $3 / 2$ & 1,2 & 462 \\
\hline${ }^{41} \mathrm{~K}$ & $1 / 2$ & $3 / 2$ & 1,2 & 254 \\
\hline${ }^{85} \mathrm{Rb}$ & $1 / 2$ & $5 / 2$ & 2,3 & 3036 \\
\hline${ }^{87} \mathrm{Rb}$ & $1 / 2$ & $3 / 2$ & 1,2 & 6835 \\
\hline
\end{tabular}

Tabela 1.1: Constituição do momento angular intrínseco de alguns átomos bosônicos de interesse, com Frequência do Espectro de transição entre os níveis hiperfinos

Então, no caso de confinamento do nível mais baixo (e.g. para ${ }^{87} \mathrm{Rb}, f=1$ ), com o auxílio de um campo magnético, pode-se fazer a separação energética dos níveis $m_{f}$. Isso é possível com as armadilhas ópticas [12], e não com as magnéticas uma vez que estas confinam apenas um dos níveis $m_{f}$, como mostrado na seção 4.2 de [8].

Antecipando o que será mostrado em mais detalhes no que segue, teremos "campos" por vezes chamado de "order parameter" para cada subnível hiperfino $\left(m_{f}\right)$, que estão relacionados com a densidade de átomos nestes níveis. Por vezes este conjunto é organizado em componentes de um vetor, sendo estas funções, passa-se a chamar este objeto de "espinor".

\footnotetext{
${ }^{2}$ Embora a configuração eletrônica mude para casos particulares como ${ }^{52} \mathrm{Cr}$.
} 
Utilizando interação com lasers podemos proporcionar acoplamento entre as componentes do espinor que descrevem o condensado. Embora recentes, já acumula uma lista crescente de temas estudados da qual fazem parte solitons [13, 14, 15], estudo de vórtices[16], verificação experimental para 2 [17] ou os 3 [18], entre outros [19, 20, 21].

\subsection{Pretensão deste trabalho}

O foco principal, o qual deve ser explorado com certo detalhe, são soluções estacionárias e sólitons em um sistema atrativo de uma dimensão(1D), nas diretrizes de [13]. Este mesmo estudo mostrou resultados partindo de formas analíticas [14], unindo a uma análise variacional.

Por consistência e completeza, será feito também uma discussão extensiva sobre o que realmente se entende como acoplamento spin-órbita, onde será evidenciado que é uma forma interpretativa de uma hamiltoniana efetiva para o sistema de bósons confinados em $f=1$, sujeitos a efeito Zeeman, sob um particular regime dos parâmetros.

Adicionalmente, estudar região onde ocorrem diferentes fases para o estado fundamental, caracterizadas pela distribuição de átomos no espeço de momentos, sendo motivado por [22, 23]. 


\section{Capítulo 2}

\section{Condensação de Bose-Einstein com}

\section{Acoplamento Spin-Órbita}

No contexto da condensação de Bose-Einstein a aproximação de Campo Médio (Mean-Field Theory) se não for a ferramenta principal, é a mais comum no estudo de Bósons a temperaturas bem próximas de zero (quantitativamente $T \ll T_{c}$ referente a (1.12)), pois como ficou sugestivo da introdução, estamos interessados num estado descrito pelo produto do estado fundamental de partícula única, ou posto de outra forma, na média dos operadores segundo este estado.

Com esta prévia, é necessário o formalismo segundo esses números de ocupação dos orbitais, descrito no que segue.

\subsection{Breve discussão de Segunda Quantização}

Como prelúdio, voltando a analisar (1.2), tomando um $\mathbf{k}_{p}$ específico, podemos ter um certo número de partículas $n_{p}$ neste estado. De tal forma que: $E=\sum_{p} n_{p} \epsilon_{p}$ com $\epsilon_{p} \doteq \hbar^{2} \mathbf{k}_{p}^{2} / 2 m$. De novo pela indistinguibilidade o que importa na caracterização da energia é a quantidade $n_{p}$ e para cada $\left|\left\{\mathbf{k}_{1}, \ldots \mathbf{k}_{n}\right\}\right\rangle$ associa-se apenas um $\left|n_{1} \ldots n_{p} \ldots\right\rangle$.

O procedimento acima estabelece um novo conjunto gerador para o mesmo espaço, considerado, assim como o anterior, ortonormalizado, cuja hamiltoniana fica representada segundo o mesmo como

$$
\mathcal{H}=\sum_{\left\{n_{i}\right\}}^{\prime}\left|n_{1} \ldots n_{l} \ldots\right\rangle\left(\sum_{p} n_{p} \epsilon_{p}\right)\left\langle n_{1} \ldots n_{l} \ldots\right|,
$$

com $\sum^{\prime}$ restrito a manter o número total de partículas. 
Contudo, isto pode ser feito para qualquer operador definido para um partícula, cujo seu espectro forme um conjunto completo. Além disso, por dizer em número de partículas é de fundamental interesse definir um operador que leva a um estado de $n \pm 1$ partículas com $n \doteq$ $\sum_{p} n_{p}$

Assumindo então que esta partícula seja "inserida" ou "retirada" segundo o número quântico $\epsilon_{l}$, encontra-se então

$$
\left\{\begin{array}{l}
a_{l}^{\dagger}\left|n_{1} \ldots n_{l} \ldots\right\rangle=c\left(n_{1}, \ldots, n_{l}, \ldots\right)\left|n_{1} \ldots n_{l}+1 \ldots\right\rangle \\
a_{l}\left|n_{1} \ldots n_{l} \ldots\right\rangle=d\left(n_{1}, \ldots, n_{l}, \ldots\right)\left|n_{1} \ldots n_{l}-1 \ldots\right\rangle
\end{array}\right.
$$

de onde um ser adjunto do outro é diretamente verificado dos coeficientes $c$ e $d$ serem não nulos e da ortogonalidade dos $\left|n_{1} \ldots n_{l} \ldots\right\rangle$. É difundida a nomenclatura de operadores aniquilação e criação para $a$ e $a^{\dagger}$, respectivamente. Representando o estado cujo $n=0$ por $|0\rangle$, convencionase $a|0\rangle \doteq \mathbf{0}$, sendo este último o elemento nulo do espaço vetorial.

A conexão com o que sabemos de Mecânica Quântica de 1 partícula é estabelecida ao detalharmos o que ocorre para o autoestado de alguma variável dinâmica(cujo autovalor é $A_{i}$, representando momento angular, posição, energia, etc) ao representar numa segunda base,

$$
a_{i}^{\dagger}|0\rangle \equiv\left|A_{i}\right\rangle=\sum_{q}\left\langle B_{q} \mid A_{i}\right\rangle\left|B_{q}\right\rangle=\sum_{q}\left\langle B_{q} \mid A_{i}\right\rangle b_{q}^{\dagger}|0\rangle .
$$

Portanto, a conversão entre a representação de operadores fica

$$
\left\{\begin{array}{l}
a_{i}^{\dagger}=\sum_{q}\left\langle B_{q} \mid A_{i}\right\rangle b_{q}^{\dagger} \\
a_{i}=\sum_{q}\left\langle A_{i} \mid B_{q}\right\rangle b_{q}
\end{array}\right.
$$

do qual um caso particular é para o operador posição

$$
\left\{\begin{aligned}
\Psi^{\dagger}(\mathbf{x}) & =\sum_{q} \psi_{q}^{*}(\mathbf{x}) b_{q}^{\dagger} \\
\Psi(\mathbf{x}) & =\sum_{q} \psi_{q}(\mathbf{x}) b_{q}
\end{aligned}\right.
$$

Surge então a expressão de "segunda" quantização, pois seria o equivalente a transformar a Função de onda em um operador, através de seus coeficientes da expansão em um conjunto completo retirado de algum operador dinâmico. 
Um fato que decorre da relação (2.4) é a invariância $\sum_{i} a_{i}^{\dagger} a_{i}=\sum_{i} b_{i}^{\dagger} b_{i}$, implicação direta de $\sum_{i}\left|A_{i}\right\rangle\left\langle A_{i}\right|=\mathbb{1}$ e $\left\langle A_{i} \mid A_{j}\right\rangle=\delta_{i j}$. A arbitrariedade das constantes em (2.2) se contorna então ao estabelecer que: $N_{i}\left|n_{1} \ldots\right\rangle=a_{i}^{\dagger} a_{i}\left|n_{1} \ldots\right\rangle=n_{i}\left|n_{1} \ldots\right\rangle$. Portanto podemos fazer a contagem total por: $N \doteq \sum_{i} a_{i}^{\dagger} a_{i} \Rightarrow N\left|n_{1} \ldots\right\rangle=n\left|n_{1} \ldots\right\rangle$ e em resumo temos

$$
\left\{\begin{aligned}
a_{l}^{\dagger}\left|n_{1} \ldots n_{l} \ldots\right\rangle & =\sqrt{n_{l}+1}\left|n_{1} \ldots n_{l}+1 \ldots\right\rangle \\
a_{l}\left|n_{1} \ldots n_{l} \ldots\right\rangle & =\sqrt{n_{l}}\left|n_{1} \ldots n_{l}-1 \ldots\right\rangle
\end{aligned}\right.
$$

Analisando a ação dos $a_{l}$ e $a_{l}^{\dagger}$ nos vetores que formam uma base $\left|n_{1} \ldots n_{l} \ldots\right\rangle$, conclui-se que, caso $i \neq l$,

$$
\begin{aligned}
& N_{i} a_{l}-a_{l} N_{i}= N_{i} a_{l}^{\dagger}-a_{l}^{\dagger} N_{i}=0, \\
& a_{l} N_{l}-N_{l} a_{l}=a_{l}, \\
& N_{l} a_{l}^{\dagger}-a_{l}^{\dagger} N_{l}=a_{l}^{\dagger} .
\end{aligned}
$$

E ainda podemos construir recursivamente a partir de $|0\rangle$ qualquer estado em número de ocupação recursivamente como

$$
\begin{aligned}
\left|n_{1} \ldots n_{l} \ldots\right\rangle & =\frac{a_{l}^{\dagger}}{\sqrt{n_{l}}}\left|n_{1} \ldots n_{l}-1 \ldots\right\rangle=\ldots=\frac{\left(a_{l}^{\dagger}\right)^{n_{l}}}{\sqrt{n_{l} !}}\left|n_{1} \ldots 0 . \ldots\right\rangle, \\
& \vdots \\
\left|n_{1} \ldots n_{l} \ldots\right\rangle & =\prod_{k=1}^{\infty} \frac{\left(a_{k}^{\dagger}\right)^{n_{k}}}{\sqrt{n_{k} !}}|0\rangle .
\end{aligned}
$$

\subsubsection{Bósons e Férmions}

A questão da algebra entre os operadores de criação e aniquilação ainda está em aberto. Um modo de fixar esta questão é assumir que a criação e/ou aniquilação reproduz o mesmo estado quântico independente da ordem. Isto é $a_{i}^{\dagger} a_{j}^{\dagger}|\Psi\rangle$ difere de $a_{j}^{\dagger} a_{i}^{\dagger}|\Psi\rangle$ por uma constante $\lambda$ tal que $|\lambda|=1$.

Este argumento de cunho heurístico, que também pode ser visto em [24] vem do estado final(após atuação dos operadores) gerado ser o mesmo, uma partícula a mais em $i / j$.

$$
a_{i}^{\dagger} a_{j}^{\dagger}-\lambda a_{j}^{\dagger} a_{i}^{\dagger}=0
$$


seja qual for a variável dinâmica associada a $a$. Considerando $\lambda \in \mathbb{R}$ leva-nos a duas opções $\lambda=$ \pm 1 que fixa uma relação de anticomutação ou comutação nula, que será atribuída a descrição de férmions ou bósons, respectivamente.

$\mathrm{O}$ mesmo se aplica em partes para a ordem entre $a$ e $a^{\dagger}$. Isto porque algumas componentes de um estado genérico $|\Psi\rangle$ na base $\left|n_{1} \ldots n_{l} \ldots\right\rangle$ exibem o contraste de $a_{l}^{\dagger} a_{l}\left|n_{1} \ldots\left(n_{l}=0\right) \ldots\right\rangle=\mathbf{0}$ e, $a_{l} a_{l}^{\dagger}\left|n_{1} \ldots\left(n_{l}=0\right) \ldots\right\rangle=\left|n_{1} \ldots\left(n_{l}=0\right) \ldots\right\rangle \neq \mathbf{0}$. Portanto

$$
a_{i} a_{i}^{\dagger}-\mu a_{i}^{\dagger} a_{i} \neq 0, \quad \mu= \pm 1
$$

Embora o argumento para (2.11) venha a se aplicar para

$$
a_{i} a_{j}^{\dagger}-\mu a_{j}^{\dagger} a_{i}=0 ; \quad i \neq j
$$

fazendo uso de (2.4) na igualdade acima, encontra-se

$$
\sum_{n m}\left\langle A_{i} \mid B_{m}\right\rangle\left\langle B_{n} \mid A_{j}\right\rangle\left(b_{m} b_{n}^{\dagger}-\mu b_{n}^{\dagger} b_{m}\right)=0
$$

Para os termos na soma em que a parte de operadores não se anula, $m=n$, podemos associar $\left(b_{m} b_{m}^{\dagger}-\mu b_{m}^{\dagger} b_{m}\right)=\Gamma$, independente de $m$ para que seja válido para todos números de ocupação já que a variável de $\left|B_{m}\right\rangle$ não foi especificada, de forma que

$$
\Gamma \sum_{m}\left\langle A_{i} \mid B_{m}\right\rangle\left\langle B_{m} \mid A_{j}\right\rangle=\Gamma\left\langle A_{i} \mid A_{j}\right\rangle=0
$$

suposto que $i \neq j$, satisfazendo assim a igualdade.

A escolha que respeita (2.6) é $\Gamma=\mathbb{1}$ para $\mu=1$, e torna-se colorário que se $\mu=-1$ a validade permanece somente se $n_{m}=0,1$. O que resta é estabelecer uma relação entre $\mu$ e $\lambda$, o que é feito por meio de (2.7) com a segunda igualdade, a direita, gerando

$$
\begin{aligned}
a_{i}^{\dagger} a_{i} a_{k}^{\dagger}-a_{k}^{\dagger} a_{i}^{\dagger} a_{i} & =0, \\
(\mu \lambda-1) a_{k}^{\dagger} a_{i}^{\dagger} a_{i} & =0, \\
\mu \lambda & =1 .
\end{aligned}
$$


Em palavras, $\mu$ e $\lambda$ devem ter o mesmo sinal. Terminamos com:

$$
\begin{array}{l|c}
\text { Bose }- \text { Einstein } & \multicolumn{1}{c}{\text { Fermi }- \text { Dirac }} \\
{\left[a_{k}, a_{l}\right]=\left[a_{k}^{\dagger}, a_{l}^{\dagger}\right]=0} & \left\{a_{k}, a_{l}\right\}=\left\{a_{k}^{\dagger}, a_{l}^{\dagger}\right\}=0 \\
{\left[a_{k}, a_{l}^{\dagger}\right]=\delta_{k l}} & \left\{a_{k}, a_{l}^{\dagger}\right\}=\delta_{k l}
\end{array}
$$

\subsubsection{Operadores de Campo e Densidade com Spin}

Como é parte essencial deste trabalho algumas palavras devem ser ditas sobre operadores compatíveis. No caso a posição não nutri relação com spin que seria um grau intrínseco interno da partícula. Isso se traduz pelo espaço vetorial ser o espaço produto $\mathcal{L}^{2}\left(\mathbb{R}^{3}, d x\right) \otimes \mathbb{C}^{n}$ onde $n=2 s+1$, dimensão para descrever spin, e os operadores comutam.

Por isso podemos simultaneamente definir autoestados de posição e spin. Isso se traduz nos operadores de criação e aniquilação pelo número quântico se desdobrar em dois (ou mais dependendo do conjunto de operadores compatíveis) $\Psi^{\dagger}(\mathbf{x}), \Psi(\mathbf{x}) \rightarrow \Psi_{\sigma}^{\dagger}(\mathbf{x}), \Psi_{\sigma}(\mathbf{x})$. Generaliza-se Então:

$$
\left\{\begin{array}{l}
\Psi_{\sigma}^{\dagger}(\mathbf{x})=\sum_{q}\left\langle\mathbf{x} \sigma \mid A_{q}\right\rangle^{*} a_{q}^{\dagger}=\sum_{q} \psi_{\sigma_{q}}^{*}(\mathbf{x}) a_{q}^{\dagger} \\
\Psi_{\sigma}(\mathbf{x})=\sum_{q}\left\langle\mathbf{x} \sigma \mid A_{q}\right\rangle a_{q}=\sum_{q} \psi_{\sigma_{q}}(\mathbf{x}) a_{q}
\end{array}\right.
$$

E a relação de completeza dos $\psi_{\sigma_{q}}$ (componentes de espinor) conduz a:

$$
\begin{array}{l|l}
\multicolumn{1}{c}{\text { Bose }- \text { Einstein }} & \multicolumn{1}{c}{\text { Fermi }- \text { Dirac }} \\
{\left[\Psi_{\sigma}(\mathbf{x}), \Psi_{\sigma^{\prime}}\left(\mathbf{x}^{\prime}\right)\right]=0} & \left\{\Psi_{\sigma}(\mathbf{x}), \Psi_{\sigma^{\prime}}\left(\mathbf{x}^{\prime}\right)\right\}=0 \\
{\left[\Psi_{\sigma}^{\dagger}(\mathbf{x}), \Psi_{\sigma^{\prime}}^{\dagger}\left(\mathbf{x}^{\prime}\right)\right]=0} & \left\{\Psi_{\sigma}^{\dagger}(\mathbf{x}), \Psi_{\sigma^{\prime}}^{\dagger}\left(\mathbf{x}^{\prime}\right)\right\}=0 \\
{\left[\Psi_{\sigma}(\mathbf{x}), \Psi_{\sigma^{\prime}}^{\dagger}\left(\mathbf{x}^{\prime}\right)\right]=\delta\left(\mathbf{x}-\mathbf{x}^{\prime}\right) \delta_{\sigma \sigma^{\prime}}} & \left\{\Psi_{\sigma}(\mathbf{x}), \Psi_{\sigma^{\prime}}^{\dagger}\left(\mathbf{x}^{\prime}\right)\right\}=\delta\left(\mathbf{x}-\mathbf{x}^{\prime}\right) \delta_{\sigma \sigma^{\prime}}
\end{array}
$$

É importante ressaltar que

$$
N_{\sigma}(\Omega) \doteq \int_{\Omega} \mathrm{d}^{3} \mathbf{x} \Psi_{\sigma}^{\dagger}(\mathbf{x}) \Psi_{\sigma}(\mathbf{x})
$$

tem como auto estado $\prod_{i=1}^{k} \Psi_{\sigma_{i}}^{\dagger}\left(\mathbf{x}_{i}\right)|0\rangle$ cujo auto valor é $\sum_{i} \delta_{\sigma \sigma_{i}} \chi_{\Omega}\left(\mathbf{x}_{i}\right)$, onde $\chi_{\Omega}$ denota a função simples que atribui 1 se $\mathbf{x} \in \Omega$ e 0 caso contrário. A verificação é imediata usando as relações de comutação e válida tanto a Bósons como Férmions. 
Portanto podemos intuitivamente definir o operador densidade por

$$
\rho_{\sigma}(\mathbf{x}) \doteq \Psi_{\sigma}^{\dagger}(\mathbf{x}) \Psi_{\sigma}(\mathbf{x})
$$

\subsubsection{Representação de Operadores}

Na dinâmica de muitos corpos podemos expressar os operadores nesse novo formalismo, em termos do que foi discutido na seção precedente. O princípio básico é representá-lo pela contagem de constituintes num autoestado enumerado $i$ multiplicada pelo seu autovalor, ou seja, $\mathcal{A}=\sum_{q} A_{q} a_{q}^{\dagger} a_{q}$, tal que $A_{q}$ são os autovalores do caso de uma partícula. Usando novamente (2.4) encontra-se

$$
\begin{aligned}
\mathcal{K} & =\sum_{q} K_{q} \sum_{p p^{\prime}} b_{p}^{\dagger} b_{p^{\prime}}\left\langle B_{p} \mid K_{q}\right\rangle\left\langle K_{q} \mid B_{p^{\prime}}\right\rangle \\
& =\sum_{p p^{\prime}} b_{p}^{\dagger} b_{p^{\prime}}\left\langle B_{p}\left|\left(\sum_{q}\left|K_{q}\right\rangle K_{q}\left\langle K_{q}\right|\right)\right| B_{p^{\prime}}\right\rangle \\
& =\sum_{p p^{\prime}} b_{p}^{\dagger} b_{p^{\prime}}\left\langle B_{p}|\hat{k}| B_{p^{\prime}}\right\rangle .
\end{aligned}
$$

Do que se traduz que precisamos somente como se comportam os números $\left\langle L_{p}|\hat{k}| L_{p^{\prime}}\right\rangle$, elementos de matriz, segundo o operador de 1 partícula $\hat{k}$. Uma generalização pode ser feita para operadores de interação chegando-se a

$$
\mathcal{V}=\frac{1}{2} \sum_{q r s t} b_{q}^{\dagger} b_{r}^{\dagger} b_{s} b_{t}\left\langle B_{q}, B_{r}|\hat{v}| B_{t}, B_{s}\right\rangle
$$

Para uma conexão com a representação no espaço de posição, fazemos a variável dinâmica $B_{q} \rightarrow \mathbf{x}, \sigma ; \sum_{q} \rightarrow \sum_{\sigma} \int \mathrm{d}^{3} \mathbf{x}$, contabilizando assim corretamente o espectro do operador. Para calcular $\left\langle\mathbf{x}^{\prime}, \sigma^{\prime}|\hat{k}| \mathbf{x}, \sigma\right\rangle$ passamos a relação de $\hat{k}$ atuando no espaço de funções: $\langle\mathbf{x}, \sigma|\hat{k}| \Phi\rangle \doteq$ $(\hat{K} \Phi)_{\sigma}(\mathbf{x})=\sum_{\sigma^{\prime}} \hat{K}_{\sigma \sigma^{\prime}} \Phi_{\sigma^{\prime}}(\mathbf{x})$ com $\Phi$ a representação em espinor de $|\Phi\rangle$. A relação anterior relembra uma multiplicação matricial, mas seus elementos podem conter derivadas, entre outras operações sobre funções.

Um conjunto de operadores um tanto geral é separável, isto é pode ser representado como 
produto $\hat{k} \equiv \hat{p} \otimes \hat{s} ;\left\langle\mathbf{x}^{\prime}, \sigma^{\prime}|\hat{k}| \mathbf{x}, \sigma\right\rangle=\left\langle\mathbf{x}^{\prime}|\hat{p}| \mathbf{x}\right\rangle\left\langle\sigma^{\prime}|\hat{s}| \sigma\right\rangle$, sendo

$$
\begin{aligned}
\hat{k}|\Phi\rangle & =\sum_{\sigma^{\prime}} \int \mathrm{d}^{3} \mathbf{x}^{\prime}\left|\mathbf{x}^{\prime} \sigma^{\prime}\right\rangle \sum_{\sigma} \hat{K}_{\sigma^{\prime} \sigma} \Phi_{\sigma}\left(\mathbf{x}^{\prime}\right) \\
& =\sum_{\sigma^{\prime}} \int \mathrm{d}^{3} \mathbf{x}^{\prime}\left|\mathbf{x}^{\prime} \sigma^{\prime}\right\rangle \sum_{\sigma} \int \mathrm{d}^{3} \mathbf{x}\left\langle\mathbf{x}^{\prime} \sigma^{\prime}|\hat{k}| \mathbf{x} \sigma\right\rangle \Phi_{\sigma}(\mathbf{x}) \\
& =\sum_{\sigma^{\prime}} \int \mathrm{d}^{3} \mathbf{x}^{\prime}\left|\mathbf{x}^{\prime} \sigma^{\prime}\right\rangle \sum_{\sigma} \int \mathrm{d}^{3} \mathbf{x}\left\langle\mathbf{x}^{\prime}|\hat{p}| \mathbf{x}\right\rangle\left\langle\sigma^{\prime}|\hat{s}| \sigma\right\rangle \Phi_{\sigma}(\mathbf{x})
\end{aligned}
$$

Além disso, podemos representar o produto $\left\langle\mathbf{x}^{\prime}|\hat{p}| f\right\rangle$, com $|f\rangle$ indicando um estado qualquer, como operações sobre funções, com este mesmo estado dado no espaço de posições $\langle\mathbf{x} \mid f\rangle=f(\mathbf{x})$ escrevendo $\hat{P} f\left(\mathbf{x}^{\prime}\right) \equiv \hat{H}_{o}\left(\mathbf{x}^{\prime}, \nabla^{\prime}\right) f\left(\mathbf{x}^{\prime}\right)=\left\langle\mathbf{x}^{\prime}|\hat{p}| f\right\rangle$. É direto estabelecer a relação $\int \mathrm{d}^{3} \mathbf{x}\left\langle\mathbf{x}^{\prime}|\hat{p}| \mathbf{x}\right\rangle f(\mathbf{x})=\hat{H}_{o}\left(\mathbf{x}^{\prime}, \nabla^{\prime}\right) f\left(\mathbf{x}^{\prime}\right)$ e concluir

$$
\hat{h}_{o}|\Phi\rangle=\sum_{\sigma^{\prime}} \int \mathrm{d}^{3} \mathbf{x}^{\prime}\left|\mathbf{x}^{\prime} \sigma^{\prime}\right\rangle \sum_{\sigma}\left\langle\sigma^{\prime}|\hat{s}| \sigma\right\rangle \hat{H}_{o}\left(\mathbf{x}^{\prime}, \nabla^{\prime}\right) \Phi_{\sigma}\left(\mathbf{x}^{\prime}\right),
$$

onde foi renomeado $\hat{k} \rightarrow \hat{h}_{o}$ por uma conveniência. Como conclusão desses passos, podemos escrever no padrão de segunda quantização um operador o qual sabe-se sua forma funcional no formalismo de Schrödinger, como

$$
\begin{aligned}
\mathcal{H}_{o} & =\sum_{p p^{\prime}} b_{p}^{\dagger} b_{p^{\prime}} \sum_{\sigma^{\prime}} \int \mathrm{d}^{3} \mathbf{x}^{\prime}\left\langle B_{p} \mid \mathbf{x}^{\prime} \sigma^{\prime}\right\rangle \sum_{\sigma}\left\langle\sigma^{\prime}|\hat{s}| \sigma\right\rangle \hat{H}_{o}\left(\mathbf{x}^{\prime}, \nabla^{\prime}\right)\left\langle\mathbf{x}^{\prime} \sigma \mid B_{p^{\prime}}\right\rangle \\
& =\int \mathrm{d}^{3} \mathbf{x}^{\prime} \sum_{\sigma^{\prime} \sigma}\left(\sum_{p}\left\langle B_{p} \mid \mathbf{x}^{\prime} \sigma^{\prime}\right\rangle b_{p}^{\dagger}\right) S_{\sigma^{\prime} \sigma} \hat{H}_{o}\left(\mathbf{x}^{\prime}, \nabla^{\prime}\right)\left(\sum_{p^{\prime}}\left\langle\mathbf{x}^{\prime} \sigma \mid B_{p^{\prime}}\right\rangle b_{p}\right) \\
\mathcal{H}_{o} & =\int \mathrm{d}^{3} \mathbf{x} \sum_{\sigma^{\prime} \sigma} \Psi_{\sigma^{\prime}}^{\dagger}(\mathbf{x}) S_{\sigma^{\prime} \sigma} \hat{H}_{o}(\mathbf{x}, \nabla) \Psi_{\sigma}(\mathbf{x}),
\end{aligned}
$$

fazendo uso de (2.24) em (2.22). Nesta nomenclatura podemos interpretar o operador acima como a hamiltoniana sem interação, onde por exemplo temos $S_{\sigma^{\prime} \sigma} \hat{H}_{o}=-\hbar^{2} \nabla^{2} / 2 m \delta_{\sigma^{\prime} \sigma}+$ $V_{\sigma^{\prime} \sigma}(\mathbf{x})$, genericamente escrevendo o termo cinético e um potencial externo ao qual todos átomos estão sujeitos. Nas seções seguintes, a intenção é construir a hamiltoniana particular de interesse para esse trabalho.

O mesmo procedimento pode ser feito para a interação, com $|\Phi\rangle$ representando um estado 
produto $\left|B_{i} B_{j}\right\rangle$

$$
\begin{aligned}
& \hat{v}|\Phi\rangle=\sum_{\sigma_{1} \sigma_{2} \sigma_{3} \sigma_{4}} \int \mathrm{d}^{3} \mathbf{x}_{1} \mathrm{~d}^{3} \mathbf{x}_{2} \mathrm{~d}^{3} \mathbf{x}_{3} \mathrm{~d}^{3} \mathbf{x}_{4}\left|\mathbf{x}_{1} \sigma_{1} ; \mathbf{x}_{2} \sigma_{2}\right\rangle\left\langle\mathbf{x}_{1} \sigma_{1} ; \mathbf{x}_{2} \sigma_{2}|\hat{v}| \mathbf{x}_{3} \sigma_{3} ; \mathbf{x}_{4} \sigma_{4}\right\rangle \\
& \left\langle\mathbf{x}_{3} \sigma_{3} ; \mathbf{x}_{4} \sigma_{4} \mid \Phi\right\rangle \\
& \hat{v}|\Phi\rangle=\sum_{\sigma_{1} \sigma_{2} \sigma_{3} \sigma_{4}} \int \mathrm{d}^{3} \mathbf{x}_{1} \mathrm{~d}^{3} \mathbf{x}_{2} \mathrm{~d}^{3} \mathbf{x}_{3} \mathrm{~d}^{3} \mathbf{x}_{4}\left|\mathbf{x}_{1} \sigma_{1} ; \mathbf{x}_{2} \sigma_{2}\right\rangle\left\langle\mathbf{x}_{1} ; \mathbf{x}_{2}\left|\hat{v}_{p}\right| \mathbf{x}_{3} ; \mathbf{x}_{4}\right\rangle \\
& \left.\hat{v}|\Phi\rangle=\sum_{\sigma_{1} \sigma_{2} \sigma_{3} \sigma_{4}} \int \sigma_{1} ; \sigma_{2}\left|\hat{v}_{s}\right| \sigma_{3} ; \sigma_{4}\right\rangle\left\langle\mathbf{x}_{3} \sigma_{3} ; \mathbf{x}_{4} \sigma_{4} \mid \Phi\right\rangle
\end{aligned}
$$

onde foi considerado novamente a separação $\hat{v}=\hat{v}_{p} \otimes \hat{v}_{s}$ ainda com $\left\langle\mathbf{x}_{1} ; \mathbf{x}_{2}\left|\hat{v}_{p}\right| \mathbf{x}_{3} ; \mathbf{x}_{4}\right\rangle=$ $V\left(\mathbf{x}_{1}, \mathbf{x}_{2}\right) \delta\left(\mathbf{x}_{1}-\mathbf{x}_{3}\right) \delta\left(\mathbf{x}_{\mathbf{2}}-\mathbf{x}_{4}\right)$.

Usando (2.23) encontramos

$$
\mathcal{V}=\frac{1}{2} \sum_{\sigma_{1} \sigma_{2} \sigma_{3} \sigma_{4}} \int \mathrm{d}^{3} \mathbf{x}_{1} \mathrm{~d}^{3} \mathbf{x}_{2} \Psi_{\sigma_{1}}^{\dagger}\left(\mathbf{x}_{1}\right) \Psi_{\sigma_{2}}^{\dagger}\left(\mathbf{x}_{2}\right) V_{\sigma_{3} \sigma_{4}}^{\sigma_{1} \sigma_{2}}\left(\mathbf{x}_{1}, \mathbf{x}_{2}\right) \Psi_{\sigma_{4}}\left(\mathbf{x}_{2}\right) \Psi_{\sigma_{3}}\left(\mathbf{x}_{1}\right)
$$

onde a notação foi abreviada com $\left\langle\sigma_{1} ; \sigma_{2}\left|\hat{v}_{s}\right| \sigma_{3} ; \sigma_{4}\right\rangle \hat{V}\left(\mathbf{x}_{1}, \mathbf{x}_{2}\right)=V_{\sigma_{3} \sigma_{4}}^{\sigma_{1} \sigma_{2}}\left(\mathbf{x}_{1}, \mathbf{x}_{2}\right)$. Genericamente por se tratarem de partículas idênticas, há simetria frente a troca de estados de spin entre o corpo 1 e 2, satisfazendo então $V_{\sigma_{4} \sigma_{3}}^{\sigma_{2} \sigma_{1}}\left(\mathbf{x}_{1}, \mathbf{x}_{2}\right)=V_{\sigma_{3} \sigma_{4}}^{\sigma_{1} \sigma_{2}}\left(\mathbf{x}_{2}, \mathbf{x}_{1}\right)$, além de depender da posição relativa, adicionalmente sendo então simétrico a $\mathrm{x}_{1} \leftrightarrow \mathrm{x}_{2}$.

\subsubsection{Dinâmica de operadores}

A dinâmica dos operadores de criação e aniquilação fica sujeito ao formalismo de Heisenberg, do qual será detalhado o operador de campo aqui. ${ }^{1}$ Começando por (2.25), temos

$$
\begin{aligned}
{\left[\Psi_{\sigma}(\mathbf{y}, t), \mathcal{H}_{o}\right] } & =\int \mathrm{d}^{3} \mathbf{x} \sum_{\sigma^{\prime \prime} \sigma^{\prime}}\left[\Psi_{\sigma}(\mathbf{y}, t), \Psi_{\sigma^{\prime \prime}}^{\dagger}(\mathbf{x}, t)\right] S_{\sigma^{\prime \prime} \sigma^{\prime}} \hat{H}_{o}(\mathbf{x}, \nabla) \Psi_{\sigma^{\prime}}(\mathbf{x}, t) \\
& =\sum_{\sigma^{\prime}} S_{\sigma \sigma^{\prime}} \hat{H}_{o}\left(\mathbf{y}, \nabla_{\mathbf{y}}\right) \Psi_{\sigma^{\prime}}(\mathbf{y}, t)
\end{aligned}
$$

\footnotetext{
${ }^{1}$ A presença do tempo como parâmetro nos operadores indicará naturalmente que se trata da representação de Heisenberg, para não adicionar mais índices
} 
E agora para (2.27) encontra-se

$$
\begin{aligned}
{\left[\Psi_{\sigma}(\mathbf{y}, t), \mathcal{V}\right]=} & \frac{1}{2} \sum_{\sigma_{1} \sigma_{2} \sigma_{3} \sigma_{4}} \int \mathrm{d}^{3} \mathbf{x}_{1} \mathrm{~d}^{3} \mathbf{x}_{2} V_{\sigma_{3} \sigma_{4}}^{\sigma_{1} \sigma_{2}}\left(\mathbf{x}_{1}, \mathbf{x}_{2}\right)\{ \\
& {\left[\Psi_{\sigma}(\mathbf{y}, t), \Psi_{\sigma_{1}}^{\dagger}\left(\mathbf{x}_{1}, t\right)\right] \Psi_{\sigma_{2}}^{\dagger}\left(\mathbf{x}_{2}, t\right) \Psi_{\sigma_{4}}\left(\mathbf{x}_{2}, t\right) \Psi_{\sigma_{3}}\left(\mathbf{x}_{1}, t\right)+} \\
& \left.\Psi_{\sigma_{1}}^{\dagger}\left(\mathbf{x}_{1}, t\right)\left[\Psi_{\sigma}(\mathbf{y}, t), \Psi_{\sigma_{2}}^{\dagger}\left(\mathbf{x}_{2}, t\right)\right] \Psi_{\sigma_{4}}\left(\mathbf{x}_{2}, t\right) \Psi_{\sigma_{3}}\left(\mathbf{x}_{1}, t\right)\right\} \\
{\left[\Psi_{\sigma}(\mathbf{y}, t), \mathcal{V}\right]=} & \sum_{\sigma_{1} \sigma_{2} \sigma_{3}} \int \mathrm{d}^{3} \mathbf{x} V_{\sigma_{2} \sigma_{3}}^{\sigma \sigma_{1}}(\mathbf{y}, \mathbf{x}) \Psi_{\sigma_{1}}^{\dagger}(\mathbf{x}, t) \Psi_{\sigma_{3}}(\mathbf{x}, t) \Psi_{\sigma_{2}}(\mathbf{y}, t)
\end{aligned}
$$

onde foi assumido a simetria frente a troca de partículas do potencial de interação, que equivale a $V_{\sigma_{3} \sigma_{4}}^{\sigma_{1} \sigma_{2}}\left(\mathbf{x}_{1}, \mathbf{x}_{2}\right)=V_{\sigma_{4} \sigma_{3}}^{\sigma_{2} \sigma_{1}}\left(\mathbf{x}_{2}, \mathbf{x}_{1}\right)$, Além de que $\Psi_{\sigma_{4}}\left(\mathbf{x}_{2}, t\right)$ e $\Psi_{\sigma_{3}}\left(\mathbf{x}_{1}, t\right)$ comutam.

Portanto a equação de "movimento" para o operador de campo fica

$$
\begin{aligned}
i \hbar \frac{d}{d t} \Psi_{\sigma}(\mathbf{y}, t)=\sum_{\sigma^{\prime}} S_{\sigma \sigma^{\prime}} \hat{H}_{o}\left(\mathbf{y}, \nabla_{\mathbf{y}}\right) \Psi_{\sigma^{\prime}}(\mathbf{y}, t)+ \\
\sum_{\sigma_{1} \sigma_{2} \sigma_{3}} \int \mathrm{d}^{3} \mathbf{x} V_{\sigma_{2} \sigma_{3}}^{\sigma \sigma_{1}}(\mathbf{y}, \mathbf{x}) \Psi_{\sigma_{1}}^{\dagger}(\mathbf{x}, t) \Psi_{\sigma_{3}}(\mathbf{x}, t) \Psi_{\sigma_{2}}(\mathbf{y}, t) .
\end{aligned}
$$

O termo que advém da interação será estudado mais a fundo na busca de uma interação efetiva que simplifica a integração presente nesta equação, mesmo assim, conduzindo ainda a uma não-linearidade.

\subsection{Interação Efetiva a baixas temperaturas}

O ponto central da interação efetiva que aqui será desenvolvida, se baseia na consideração de que a energia envolvida no espalhamento é muito pequena, qualitativamente, em decorrência de $T \rightarrow 0$. Supondo o potencial função de $\left|\mathbf{x}_{1}-\mathbf{x}_{2}\right|$ partimos da equação de Lippmann-Schwinger

$$
|E\rangle=|\mathbf{p}\rangle+\left(E-\frac{\hat{\mathbf{p}}^{2}}{2 \mu}+i \epsilon\right)^{-1} V|E\rangle
$$

onde $\mu=m_{1} m_{2} /\left(m_{1}+m_{2}\right)$ é a massa reduzida e $|\mathbf{p}\rangle$ é autoestado do operador de energia cinética relativo $\hat{\mathbf{p}}^{2} / 2 \mu$, o qual ainda carrega o grau de spin total que deve ser tomado como condição antes da colisão. Após inserir algumas projeções, mostra-se que

$$
\psi_{\Sigma}^{E}(\mathbf{x})=A_{\Sigma} \frac{\mathrm{e}^{i \mathbf{k} \cdot \mathbf{x}}}{(2 \pi \hbar)^{3 / 2}}+\int \mathrm{d}^{3} \mathbf{x}^{\prime} \sum_{\Sigma^{\prime}} \int \mathrm{d}^{3} \mathbf{p} \frac{\langle\mathbf{x} \mid \mathbf{p}\rangle\left\langle\mathbf{p} \mid \mathbf{x}^{\prime}\right\rangle}{E-\frac{p^{2}}{2 \mu}+i \epsilon}\left\langle\Sigma\left|\hat{v}_{s}\right| \Sigma^{\prime}\right\rangle \hat{V}\left(\mathbf{x}^{\prime}\right) \psi_{\Sigma^{\prime}}^{E}\left(\mathbf{x}^{\prime}\right)
$$


sendo $k=\sqrt{2 E \mu} / \hbar$ fixado pela energia do movimento relativo $E, \Sigma$ a projeção do spin sobre um eixo de quantização e $\psi_{\Sigma}^{E}(\mathbf{x}) \equiv\langle\mathbf{x} \Sigma \mid E\rangle$ a componente $\Sigma$ do spinor deste auto estado. A integral no espaço de momento pode ser feita por método de resíduos e fornece

$$
\psi_{\Sigma}^{E}(\mathbf{x})=N_{\Sigma} \mathrm{e}^{i \mathbf{k} \cdot \mathbf{x}}-\frac{2 \mu}{\hbar^{2}} \sum_{\Sigma^{\prime}}\left\langle\Sigma\left|\hat{v}_{s}\right| \Sigma^{\prime}\right\rangle \int \mathrm{d}^{3} \mathbf{x}^{\prime} \frac{\mathrm{e}^{i k\left|\mathbf{x}-\mathbf{x}^{\prime}\right|}}{4 \pi\left|\mathbf{x}-\mathbf{x}^{\prime}\right|} \hat{V}\left(\mathbf{x}^{\prime}\right) \psi_{\Sigma^{\prime}}^{E}\left(\mathbf{x}^{\prime}\right)
$$

Usualmente o potencial tem um certo alcance, quantitativamente, deve decrescer mais rápido que o Coulombiano $\lim _{r \rightarrow 0} r V(r)=0$ (detalhes em [25] cap. 3.6). Esta condição é perfeitamente satisfeita para colisões atômicas que temos interesse. É possível justificar a grandeza alcance de potencial a medida que se torna desprezível frente ao termo centrífugo da equação de Schrödinger, a ser denotado por " $r_{o}$ "

Por potencial efetivo, buscamos um que reproduza a seção de choque, e para esta devemos analisar (2.33) em $|\mathbf{x}| \gg\left|\mathbf{x}^{\prime}\right| \Rightarrow\left|\mathbf{x}-\mathbf{x}^{\prime}\right| \approx|\mathbf{x}|-\hat{\mathbf{r}} \cdot \mathbf{x}^{\prime}$, com $\mathbf{k}^{\prime} \doteq k \hat{\mathbf{r}}$, levando a

$$
\begin{aligned}
\psi_{\Sigma}^{E}(\mathbf{x}) & =N_{\Sigma}\left[\mathrm{e}^{i \mathbf{k} \cdot \mathbf{x}}+\frac{\mathrm{e}^{i k r}}{r} f_{\Sigma}\left(\mathbf{k}, \mathbf{k}^{\prime}\right)\right] \\
\operatorname{com} & f_{\Sigma}\left(\mathbf{k}, \mathbf{k}^{\prime}\right)=-\frac{2 \mu}{4 \pi \hbar^{2}} \sum_{\Sigma^{\prime}}\left\langle\Sigma\left|\hat{v}_{s}\right| \Sigma^{\prime}\right\rangle \int \mathrm{d}^{3} \mathbf{x}^{\prime} \mathrm{e}^{-i \mathbf{k}^{\prime} \cdot \mathbf{x}^{\prime}} \hat{V}\left(\mathbf{x}^{\prime}\right) \frac{\psi_{\Sigma^{\prime}}^{E}\left(\mathbf{x}^{\prime}\right)}{N_{\Sigma}}
\end{aligned}
$$

\subsubsection{Aproximação de Born e um critério de validade}

A equação integral acima admite solução perturbativa substituindo recursivamente $\psi_{\Sigma^{\prime}}^{E}\left(\mathbf{x}^{\prime}\right)$. A aproximação de Born é o primeiro termo dessa recorrência. Para sua validade uma condição necessária é que de fato $\psi_{\Sigma}^{E}(\mathbf{x}) \approx N_{\Sigma} \mathrm{e}^{i \mathbf{k} \cdot \mathbf{x}}$, ao menos no domínio onde o potencial não se anula. $^{2}$

Tomando um volume onde o potencial é não nulo, uma forma de assegurar isso é exigir que

$$
\begin{aligned}
\left|\frac{\Psi_{\Sigma}^{E}\left(\alpha \mathbf{r}_{o}\right)-N_{\Sigma} \mathrm{e}^{i \alpha \mathbf{k} \cdot \mathbf{r}_{o}}}{N_{\Sigma} \mathrm{e}^{i \alpha \mathbf{k} \cdot \mathbf{r}_{o}}}\right| & \ll 1 ; 0<\alpha<1, \mathbf{r}_{o} \doteq r_{o} \hat{\mathbf{r}} \\
\Rightarrow \int_{\hat{V} \neq 0} \mathrm{~d}^{3} \mathbf{x}\left|\frac{\Psi_{\Sigma}^{E}\left(\alpha \mathbf{r}_{o}\right)-N_{\Sigma} \mathrm{e}^{i \alpha \mathbf{k} \cdot \mathbf{r}_{o}}}{N_{\Sigma} \mathrm{e}^{i \alpha \mathbf{k} \cdot \mathbf{r}_{o}}}\right| & \ll \frac{4 \pi r_{o}^{3}}{3} .
\end{aligned}
$$

Fazendo uso de (2.34) neste última, adquire-se a expressão

$$
\frac{2 \mu}{4 \pi \hbar^{2}} \int_{\Omega} \frac{\mathrm{d}^{3} \mathbf{x}}{r}\left|\int \mathrm{d}^{3} \mathbf{x}^{\prime} \mathrm{e}^{-i\left(\mathbf{k}^{\prime}-\mathbf{k}\right) \cdot \mathbf{x}^{\prime}} \hat{V}\left(\mathbf{x}^{\prime}\right) \sum_{\Sigma^{\prime}}\left\langle\Sigma\left|\hat{v}_{s}\right| \Sigma^{\prime}\right\rangle \frac{A_{\Sigma^{\prime}}}{A_{\Sigma}}\right| \ll \frac{4 \pi r_{o}^{3}}{3} .
$$

\footnotetext{
${ }^{2}$ Mesmo que seja bem diferente de $N_{\Sigma} \mathrm{e}^{i \mathbf{k}} \cdot \mathbf{x}$ fora do alcance do potencial, a contribuição na integração é nula já que em boa aproximação $V=0$ nessa região por definição
} 
O que antes foi enunciado qualitativamente, podemos enunciar quantitativamente. Espalhamento em baixas energias significa a condição

$$
k r_{o} \ll 1 ; \quad k \doteq \sqrt{2 \mu E} / \hbar,
$$

ou seja, o comprimento de onda da partícula livre antes da colisão bem maior que o raio de alcance do potencial. Portanto justifica-se, em primeira ordem, $\mathrm{e}^{-i\left(\mathbf{k}^{\prime}-\mathbf{k}\right) \cdot \mathbf{x}^{\prime}} \approx 1$. Substituindo o valor da integral em $\mathrm{d}^{3} \mathbf{x}$ e utilizando o valor médio para integrais, na referente a $\mathrm{d}^{3} \mathbf{x}^{\prime}$, depara-se com

$$
\begin{aligned}
\frac{\mu r_{o}^{2}}{\hbar^{2}} \mid \sum_{\Sigma^{\prime}}\left\langle\Sigma\left|\hat{v}_{s}\right| \Sigma^{\prime}\right\rangle \overline{\hat{V}\left(\mathbf{x}^{\prime}\right)} & \mid \frac{4 \pi r_{o}^{3} \in \Omega}{3} \ll \frac{4 \pi r_{o}^{3}}{3} \\
\frac{\mu r_{o}^{2}}{\hbar^{2}}\left|\sum_{\Sigma^{\prime}}\left\langle\Sigma\left|\hat{v}_{s}\right| \Sigma^{\prime}\right\rangle \overline{\hat{V}\left(\mathbf{x}^{\prime}\right)_{x^{\prime} \in \Omega}}\right| & \ll 1 .
\end{aligned}
$$

A interação entre átomos embora difícil de se obter uma forma explícita, dificilmente não se enquadra na condição acima, além de que $r_{o}$ é tipicamente da ordem do raio atômico.

Deve-se ainda notar que para que faça sentido a reprodução da seção de choque por um potencial efetivo, devemos ter um sistema não muito denso, caso contrário, a hipótese feita parágrafos acima de $|\mathbf{x}| \gg\left|\mathbf{x}^{\prime}\right|$ não poderia ser satisfeita já que poderia ocorrer a colisão sucessiva com outro átomo.

\subsection{2 "Scattering Lenght"}

Retrocedendo a (2.34) com a aproximação de Born e fazendo uso de (2.37)

$$
f_{\Sigma}\left(\mathbf{k}, \mathbf{k}^{\prime}\right)=-\frac{2 \mu}{4 \pi \hbar^{2}} \sum_{\Sigma^{\prime}}\left\langle\Sigma\left|\hat{v}_{s}\right| \Sigma^{\prime}\right\rangle \int \mathrm{d}^{3} \mathbf{x}^{\prime} \hat{V}\left(\mathbf{x}^{\prime}\right) \frac{N_{\Sigma^{\prime}}}{N_{\Sigma}}
$$

o qual passa a independer de $\mathbf{k}, \mathbf{k}^{\prime}$. Com interação que conserva a polarização de spin total, ou seja, $\left\langle\Sigma\left|\hat{v}_{s}\right| \Sigma^{\prime}\right\rangle=v_{\Sigma} \delta_{\Sigma \Sigma^{\prime}}$ reduz-se o número de espalhamento possíveis. Define-se portanto, $\operatorname{com} \mu=m / 2$ pois as massas são iguais, a quantia

$$
a_{\Sigma}=\frac{m}{4 \pi \hbar^{2}} \sum_{\Sigma^{\prime}}\left\langle\Sigma\left|\hat{v}_{s}\right| \Sigma^{\prime}\right\rangle \int \mathrm{d}^{3} \mathbf{x}^{\prime} \hat{V}\left(\mathbf{x}^{\prime}\right)
$$

Esta definição é fundamental, pois mesmo sem conhecer a forma explícita do potencial, 
podemos trabalhar usando $a_{\Sigma}$ como um parâmetro na equação. Um potencial efetivo é aquele que reproduz estas condições de espalhamento e, pode ser feito

$$
\left\langle\Sigma\left|\hat{v}_{s}\right| \Sigma^{\prime}\right\rangle \hat{V}\left(\mathbf{x}_{1}, \mathbf{x}_{2}\right)=\left(\frac{4 \pi \hbar^{2}}{m} a_{\Sigma}\right) \delta_{\Sigma \Sigma^{\prime}} \delta\left(\mathbf{x}_{1}-\mathbf{x}_{2}\right)
$$

Notemos que $a_{\Sigma}$ simplesmente enumera estados de spin, que pode ser tratado como total ou no espaço produto $|\Sigma\rangle \rightarrow\left|\sigma_{1}\right\rangle\left|\sigma_{2}\right\rangle$, o que fornece a equação acima levemente modificada para

$$
\left\langle\sigma_{1} ; \sigma_{2}\left|\hat{v}_{s}\right| \sigma_{3} ; \sigma_{4}\right\rangle \hat{V}\left(\mathbf{x}_{1}, \mathbf{x}_{2}\right)=V_{\sigma_{3} \sigma_{4}}^{\sigma_{1} \sigma_{2}}\left(\mathbf{x}_{1}, \mathbf{x}_{2}\right)=\left(\frac{4 \pi \hbar^{2}}{m} a_{\sigma_{1} \sigma_{2}}\right) \delta_{\sigma_{1} \sigma_{3}} \delta_{\sigma_{2} \sigma_{4}} \delta\left(\mathbf{x}_{1}-\mathbf{x}_{2}\right)
$$

Voltando a analisar a função de onda em termos da distância relativa $r$ encontra-se

$$
\Psi_{\Sigma}^{E}(\mathbf{r})=N_{\Sigma}\left[\mathrm{e}^{i \mathbf{k} \cdot \mathbf{r}}-\mathrm{e}^{i k r} \frac{a_{\Sigma}}{r}\right]
$$

a qual volta a se aproximar de partícula livre quando $r \gg a_{\Sigma}$. Portanto a ideia de comprimento de espalhamento fornece uma análise quantitativa onde a função de onda é efetivamente diferente de uma partícula livre, de outra forma a região na qual o potencial a afeta.

A não descrição de interação de 3 corpos nos exige a condição que o espaçamento médio entre partículas $(\bar{d})$ deve portanto satisfazer $\bar{d} \gg a_{\Sigma}$ ou de forma equivalente, a densidade típica(média) obedece $\bar{\rho} \equiv 1 / \bar{d}^{3} \Rightarrow \bar{\rho} a_{\Sigma}^{3} \ll 1$. Esta condição é fundamental para a teoria de Campo Médio pelas colisões serem escassas e a ocupação dos orbitais excitados desprezível.

Fica estabelecida a seguinte identidade de operação, reescalonando $g_{\sigma_{1} \sigma_{2}} \doteq 4 \pi \hbar^{2} a_{\sigma_{1} \sigma_{2}} / m$ segundo (2.42), obtendo para (2.30) a forma

$$
\begin{aligned}
i \hbar \frac{d}{d t} \Psi_{\sigma}(\mathbf{y}, t)=\sum_{\sigma^{\prime}} S_{\sigma \sigma^{\prime}} \hat{H}_{o}\left(\mathbf{y}, \nabla_{\mathbf{y}}\right) \Psi_{\sigma^{\prime}}(\mathbf{y}, t)+ \\
\\
\left.\qquad \sum_{\sigma^{\prime}} g_{\sigma \sigma^{\prime}} \Psi_{\sigma^{\prime}}^{\dagger}(\mathbf{y}, t) \Psi_{\sigma^{\prime}}(\mathbf{y}, t)\right] \Psi_{\sigma}(\mathbf{y}, t) .
\end{aligned}
$$

\subsection{Descrição de Campo Médio}

Novamente um condensado de Bose-Einstein se caracteriza pela energia mínima que o sistema possa ter. A descrição de campo médio, estabelece que os operadores são aproximadamente descritos por seus valores médios segundo um certo estado e, o que caberá aqui é argumentar sobre este estado. 
Um estado arbitrário de $N$ partículas evolui, segundo a equação de Schrödinger

$$
i \hbar \frac{\partial\left|\psi_{t}^{(N)}\right\rangle}{\partial t}=\left(\mathcal{H}_{o}+\mathcal{V}\right)\left|\psi_{t}^{(N)}\right\rangle
$$

Nesta ocasião um observável do sistema pode ser medido através de $\left\langle\psi_{t}^{(N)}|\mathcal{A}| \psi_{t}^{(N)}\right\rangle$, embora não há uma forma explícita para esta solução.

Voltando as condições, como já mencionado em um sistema dito “diluto”, com espaçamento médio de partículas maior em algumas ordens de grandeza que o comprimento de espalhamento, podemos inferir que as interações não são frequentes na evolução temporal.

Analisando (2.23), a parte de interação, pode causar excitações dos estados individuais dos átomos, que por não serem frequentes, a contagem do estado fundamental deve ser muito maior do que qualquer outro, no decorrer da dinâmica.

Em palavras, a evolução temporal de um condensado de Bose-Einstein, permanece um condensado, significando assim que é desprezível a contagem de constituintes em estados excitados(depleção do estado fundamental).

Portanto prosseguimos com a seguinte idealização, de que possamos definir um estado para um certo instante $t$ dado pelo produto dos estados individuais que minimizam a energia do sistema(por não haver a possibilidade de encontrar o estado fundamental das $\mathrm{N}$ partículas)

$$
|\mathcal{M F}(N), t\rangle \doteq \frac{\left(a_{o}^{\dagger}(t)\right)^{N}}{\sqrt{N !}}|0\rangle
$$

onde recordando da (2.3) agora generalizada para $a_{o}^{\dagger}(t)|0\rangle \doteq\left|\epsilon_{o}, t\right\rangle$ descreve orbitais individuais dependente do tempo. As (2.4) se generalizam, com relação aos operadores de campo, para:

$$
\left\{\begin{array}{l}
a_{o}^{\dagger}(t)=\sum_{\sigma} \int \mathrm{d} \mathbf{x}\left\langle\mathbf{x}, \sigma \mid \epsilon_{o}, t\right\rangle \Psi_{\sigma}^{\dagger}(\mathbf{x}) \\
a_{o}(t)=\sum_{\sigma} \int \mathrm{d} \mathbf{x}\left\langle\epsilon_{o}, t \mid \mathbf{x}, \sigma\right\rangle \Psi_{\sigma}(\mathbf{x})
\end{array}\right.
$$

Reciprocamente, obtém-se:

$$
\left\{\begin{array}{l}
\Psi_{\sigma}^{\dagger}(\mathbf{x})=\sum_{q} \phi_{\sigma}^{(q) *}(\mathbf{x}, t) a_{q}^{\dagger}(t) \\
\Psi_{\sigma}(\mathbf{x})=\sum_{q} \phi_{\sigma}^{(q)}(\mathbf{x}, t) a_{q}(t)
\end{array}\right.
$$


Sendo daqui em diante $\phi_{\sigma}^{(q)}(\mathbf{x}, t) \doteq\left\langle\mathbf{x}, \sigma \mid \epsilon_{q}, t\right\rangle$, e $\psi^{(N)}(\mathbf{x}, t) \equiv \sqrt{N} \phi_{\sigma}^{(o)}(\mathbf{x}, t)$, onde este último traça relação com a densidade de partículas, pois retomando as relações de comutação $\left[a_{j}(t), a_{i}^{\dagger}(t)\right]=\delta_{i j}$ temos

$$
\begin{aligned}
\Psi_{\sigma}(\mathbf{x})|\mathcal{M F}(N), t\rangle & =\psi^{(N)}(\mathbf{x}, t)|\mathcal{M F} \mathcal{F}(N-1), t\rangle \\
\left\langle\mathcal{M F}(N), t\left|\Psi_{\sigma}^{\dagger}(\mathbf{x}) \Psi_{\sigma}(\mathbf{x})\right| \mathcal{M F}(N), t\right\rangle & =\left|\psi^{(N)}(\mathbf{x}, t)\right|^{2}
\end{aligned}
$$

Daqui segue-se o mesmo princípio que estabelece a equação de Schrödinger, onde exigirse-á que a escolha de $\psi^{(N)}(\mathbf{x}, t)$ minimize a energia, incluindo a dependência temporal, levando ao princípio variacional dependente do tempo. Isto é feito por intermédio da derivada funcional se anular [26, cap. 33] para o funcional

$$
\begin{aligned}
\frac{\delta}{\delta \phi_{\beta}^{(o) *}\left(\mathbf{x}^{\prime}, t^{\prime}\right)} \int \mathrm{d} t\{i \hbar\langle\mathcal{M F}(N), t| & \left(\frac{\mathrm{d}}{\mathrm{d} t}|\mathcal{M} \mathcal{F}(N), t\rangle\right)- \\
& \left.\left\langle\mathcal{M} \mathcal{F}(N), t\left|\left(\mathcal{H}_{o}+\mathcal{V}\right)\right| \mathcal{M} \mathcal{F}(N), t\right\rangle\right\}=0 .
\end{aligned}
$$

Para o primeiro termo temos

$$
\begin{aligned}
\frac{\mathrm{d}}{\mathrm{d} t}|\mathcal{M F}(N), t\rangle & =\sqrt{N} \frac{\left(a_{o}^{\dagger}(t)\right)^{N-1}}{\sqrt{(N-1) !}} \frac{\mathrm{d} a_{o}^{\dagger}(t)}{\mathrm{d} t}|0\rangle, \\
\frac{\mathrm{d} a_{o}^{\dagger}(t)}{\mathrm{d} t} & =\sum_{\sigma} \int \mathrm{d}^{3} \mathbf{x} \frac{\partial \phi_{\sigma}^{(o)}(\mathbf{x}, t)}{\partial t} \Psi_{\sigma}^{\dagger}(\mathbf{x}),
\end{aligned}
$$

que juntos conduzem a

$$
\begin{aligned}
& \langle\mathcal{M F}(N), t|\left(\frac{\mathrm{d}}{\mathrm{d} t}|\mathcal{M} \mathcal{F}(N), t\rangle\right)= \\
& \sum_{\sigma} \int \mathrm{d} \mathbf{x} \frac{\partial \sqrt{N} \phi_{\sigma}^{(o)}(\mathbf{x}, t)}{\partial t}\left\langle\mathcal{M F}(N), t\left|\Psi_{\sigma}^{\dagger}(\mathbf{x})\right| \mathcal{M} \mathcal{F}(N-1), t\right\rangle,
\end{aligned}
$$

e adicionalmente atuando com $\Psi_{\sigma}^{\dagger}(\mathbf{x})$ a esquerda, consegue-se

$$
\langle\mathcal{M} \mathcal{F}(N), t|\left(\frac{\mathrm{d}}{\mathrm{d} t}|\mathcal{M} \mathcal{F}(N), t\rangle\right)=\sum_{\sigma} \int \mathrm{d}^{3} \mathbf{x} \psi_{\sigma}^{(N) *}(\mathbf{x}, t) \frac{\partial \psi_{\sigma}^{(N)}(\mathbf{x}, t)}{\partial t}
$$

E lançando (2.49) para ser usada em (2.25) e (2.27) respectivamente, permite expressar o 
Funcional (2.51) em termos de $\psi$ como

$$
\begin{aligned}
& \frac{\delta}{\delta \phi_{\beta}^{(o) *}\left(\mathbf{x}^{\prime}, t^{\prime}\right)} \int \mathrm{d}^{3} \mathbf{x} \mathrm{d} t\left\{i \hbar \sum_{\sigma \sigma^{\prime}} \int \mathrm{d}^{3} \mathbf{x} \psi_{\sigma}^{(N) *}(\mathbf{x}, t) \frac{\partial \psi_{\sigma}^{(N)}(\mathbf{x}, t)}{\partial t}-\right. \\
& \left.\psi_{\sigma^{\prime}}^{(N) *}(\mathbf{x}, t) S_{\sigma^{\prime} \sigma} \hat{H}_{o}(\mathbf{x}, \nabla) \psi_{\sigma}^{(N)}(\mathbf{x}, t)-\frac{1}{2} g_{\sigma^{\prime} \sigma}\left|\psi_{\sigma^{\prime}}^{(N)}(\mathbf{x}, t)\right|^{2}\left|\psi_{\sigma}^{(N-1)}(\mathbf{x}, t)\right|^{2}\right\}=0 .
\end{aligned}
$$

Utilizando

$$
\frac{\delta}{\delta \phi_{\beta}^{(o) *}\left(\mathbf{x}^{\prime}, t^{\prime}\right)} \psi_{\sigma}^{(N) *}(\mathbf{x}, t)=\sqrt{N} \delta^{3}\left(\mathbf{x}-\mathbf{x}^{\prime}\right) \delta\left(t-t^{\prime}\right) \delta_{\sigma \beta},
$$

obtemos

$$
i \hbar \frac{\partial \phi_{\sigma}^{(o)}(\mathbf{x}, t)}{\partial t}=\sum_{\sigma^{\prime}} S_{\sigma \sigma^{\prime}} \hat{H}_{o}(\mathbf{x}, \nabla) \phi_{\sigma^{\prime}}^{(o)}(\mathbf{x}, t)+(N-1) g_{\sigma \sigma^{\prime}}\left|\phi_{\sigma^{\prime}}^{(o)}(\mathbf{x}, t)\right|^{2} \phi_{\sigma}^{(o)}(\mathbf{x}, t)
$$

Para um número suficientemente alto de constituintes podemos fazer $N-1 \simeq N$ além de assumir que a densidade não muda para a diferença de uma partícula(tipicamente $N \sim$ $\left.10^{4}, 10^{5}\right)$. Portanto $\psi_{\sigma}^{(N)}(\mathbf{x}, t) \simeq \psi_{\sigma}^{(N-1)}(\mathbf{x}, t) \equiv \psi_{\sigma}(\mathbf{x}, t)$, resultando em

$$
i \hbar \frac{\partial \psi_{\sigma}(\mathbf{x}, t)}{\partial t}=\sum_{\sigma^{\prime}} S_{\sigma \sigma^{\prime}} \hat{H}_{o}(\mathbf{x}, \nabla) \psi_{\sigma^{\prime}}(\mathbf{x}, t)+\left[\sum_{\sigma^{\prime}} g_{\sigma \sigma^{\prime}}\left|\psi_{\sigma^{\prime}}(\mathbf{x}, t)\right|^{2}\right] \psi_{\sigma}(\mathbf{x}, t)
$$

A equação acima é de suma importância e é usual em toda literatura sobre o assunto, conhecida como equação de Gross-Pitaevskii(EGP). Determina a evolução temporal do que alguns nomeiam "order parameter" ou "Função de Onda do Condensado", cujo módulo quadrado fornece a densidade do condensado para $T \ll T_{c}$.

Alguns conceitos devem ser concretizados no que foi excluído da descrição. Tudo se baseou na negligência do número de partículas com energia acima da fundamental na ordem mais baixa de aproximação. Isso significa excluir efeitos de depleção do estado fundamental tanto por temperatura como por interação.

A energia também passa a depender desses novos parâmetros $\phi_{\sigma}(\mathbf{x}, t), \forall \sigma=-f, \ldots, f$ numerados pelos estados de spin, e omitido o índice superior ${ }^{(o)}$. A saber, fica determinada pelo 
valor médio segundo $|\mathcal{M F}(N), t\rangle$ que é o segundo termo de (2.56)

$$
\begin{array}{rl}
\mathcal{E}\left[\phi_{-f}^{*}, \ldots, \phi_{f}^{*}, \phi_{-f}, \ldots, \phi_{f}, N\right]=\sum_{\sigma \sigma^{\prime}} \int \mathrm{d}^{3} \mathbf{x} & N \phi_{\sigma}(\mathbf{x}, t) S_{\sigma \sigma^{\prime}} \hat{H}_{o}(\mathbf{x}, \nabla) \phi_{\sigma}(\mathbf{x}, t)- \\
\frac{N(N-1)}{2} g_{\sigma \sigma^{\prime}}\left|\phi_{\sigma^{\prime}}(\mathbf{x}, t)\right|^{2}\left|\phi_{\sigma}(\mathbf{x}, t)\right|^{2} .
\end{array}
$$

Voltando a enfatizar que podemos nos livrar dos índices $N$ e $N-1$ na justificativa que a diferença de uma partícula não influi a densidade, e poder escrever em termos de $\psi$ simplesmente, estando implícito o número de constituintes na normalização.

\subsubsection{Estados Estacionários}

Por estado estacionário entende-se que a densidade não evolui temporalmente, $\left|\psi_{\sigma}^{(N)}(\mathbf{x}, t)\right| \equiv$ $\left|\psi_{\sigma}^{(N)}(\mathbf{x})\right|$, da onde a forma mais geral, respeitando (2.58), é $\phi_{\sigma}(\mathbf{x}, t)=\sqrt{\rho_{\sigma}(\mathbf{x})} \mathrm{e}^{-i \mu t / \hbar} \mathrm{com}$ $\sum_{\sigma} \int \mathrm{d}^{3} \mathbf{x} \rho_{\sigma}(\mathbf{x})=1$. Esta constante $\mu$ a aparecer é o Potencial Químico, pois ao manipular $\operatorname{com} \sum_{\sigma} \int \mathrm{d}^{3} \mathbf{x} \phi_{\sigma}^{*}(\mathbf{x}) \times(2.58)$, produz

$$
\begin{aligned}
\mu & =\int \mathrm{d}^{3} \mathbf{x}\left\{\sum_{\sigma \sigma^{\prime}} \sqrt{\rho_{\sigma}(\mathbf{x})} S_{\sigma \sigma^{\prime}} \hat{H}_{o}(\mathbf{x}, \nabla) \sqrt{\rho_{\sigma^{\prime}}(\mathbf{x})}+(N-1) g_{\sigma \sigma^{\prime}} \rho_{\sigma^{\prime}}(\mathbf{x}) \rho_{\sigma}(\mathbf{x})\right\} \\
& =\mathcal{E}\left[\phi_{\vec{\sigma}}, \phi_{\vec{\sigma}}^{*}, N+1\right]-\mathcal{E}\left[\phi_{\vec{\sigma}}, \phi_{\vec{\sigma}}^{*}, N\right] \approx \frac{\partial \mathcal{E}\left[\psi_{\vec{\sigma}}^{(N)}, \psi_{\vec{\sigma}}^{*(N)}\right]}{\partial N}
\end{aligned}
$$

que é facilmente identificado a partir dos conceitos da Termodinâmica. Por completeza, a EGP. para estados estacionários fica então dada por

$$
\mu \psi_{\sigma}(\mathbf{x})=\sum_{\sigma^{\prime}} S_{\sigma \sigma^{\prime}} \hat{H}_{o}(\mathbf{x}, \nabla) \psi_{\sigma^{\prime}}(\mathbf{x})+\left[\sum_{\sigma^{\prime}} g_{\sigma \sigma^{\prime}}\left|\psi_{\sigma^{\prime}}(\mathbf{x})\right|^{2}\right] \psi_{\sigma}(\mathbf{x})
$$

onde $\psi_{\sigma}(\mathbf{x}) \equiv \sqrt{N \rho_{\sigma}(\mathbf{x})}$.

\subsubsection{Ação do Campo}

As equações de movimentos obtidas em (2.59) podem ser igualmente encontradas utilizando princípios variacionais sobre um funcional de ação, caso seja definido em função dos campos 
enumerados por $\sigma$ dados por $\psi_{\sigma}$ a Lagrangiana

$$
L(t)=\sum_{\sigma} \int \mathrm{d}^{3} \mathbf{x} \frac{i \hbar}{2}\left[\psi_{\sigma}^{*}(\mathbf{x}, t) \frac{\partial \psi_{\sigma}}{\partial t}-\psi_{\sigma}(\mathbf{x}, t) \frac{\partial \psi_{\sigma}^{*}}{\partial t}\right]-\mathcal{E}
$$

onde

$$
\mathcal{E}=\sum_{\sigma \sigma^{\prime}} \int \mathrm{d}^{3} \mathbf{x} \psi_{\sigma}(\mathbf{x}, t) S_{\sigma \sigma^{\prime}} \hat{H}_{o}(\mathbf{x}, \nabla) \psi_{\sigma}(\mathbf{x}, t)-\frac{1}{2} g_{\sigma \sigma^{\prime}}\left|\psi_{\sigma^{\prime}}(\mathbf{x}, t)\right|^{2}\left|\psi_{\sigma}(\mathbf{x}, t)\right|^{2}
$$

e então o funcional dito ação definido por

$$
\mathcal{S}(t)=\int_{-\infty}^{\infty} \mathrm{d} t L(t)
$$

Esta formulação é também descrita em [8, cap. 7], e será útil ao tratar o problema da presente dissertação, uma vez que é a base de cálculo variacional para explorar a dinâmica do sistema [14].

\subsubsection{Soluções do tipo soliton}

Dentre as diversas soluções que uma Equação diferencial parcial pode ter, pode-se procurar por aquelas que a dependência espaço-temporal se dá $\psi(\mathbf{x}, t)=f(\mathbf{n} \cdot \mathbf{x}-v t)$, conhecida da equação de onda. Neste caso, para todo instante pode-se encontrar $\mathbf{x}^{\prime}$ tal que $\mathbf{n} \cdot \mathbf{x}^{\prime}=v t+\mathbf{n} \cdot \mathbf{x}$ e forneça $\psi\left(\mathbf{x}^{\prime}, t\right)=\psi(\mathbf{x}, 0)$, portanto a função só desloca sua forma inicial.

Contudo, o perfil de solução mencionado acima é somente um caso particular de ondas solitárias. Estas não necessariamente se propagam com velocidade constante, e no geral estão restritas a uma localidade, ou seja, possuem um máximo ou mínimo local. Isto caracteriza dois tipos, com máximo("soliton brilhante") ou mínimo("soliton escuro") local e uma forma assintótica uniforme, $\psi(\mathbf{x}, 0) \rightarrow 0,|\mathbf{x}| \rightarrow \infty$ ou $\psi(\mathbf{x}, 0) \rightarrow c>\psi(0,0)$, respectivamente, em cada caso, considerado centrado em zero tais pontos críticos.

Para um condensado estamos por vezes interessados nesse tipo de solução para a densidade, parcial $\left|\psi_{\sigma}(\mathbf{x}, t)\right|^{2}$ ou total $\sum_{\sigma}\left|\psi_{\sigma}(\mathbf{x}, t)\right|^{2}$. Isso significa para um soliton brilhante, que os átomos permanecem numa região confinada do espaço e são dito estarem auto-confinados, caso tal solução seja possível na ausência de armadilha.

Portanto, adjetiva-se uma solução como soliton brilhante se: 
1. $f(\mathbf{x}, t)=f(\mathbf{x}-g(t), 0)$;

2. Localidade, i.e $f(\mathbf{x}, 0) \rightarrow 0,|\mathbf{x}| \rightarrow \infty$;

onde na listagem acima $f$ pode representar a densidade total ou parcial. Um modo de abordar o problema é partir de formas analíticas para uma escolha específica dos parâmetros das equações, tornar as constantes em variáveis e assim usar princípios variacionais a partir de uma ação para o campo [14].

\subsection{Condensado na presença de Lasers}

Recapitulando a tabela 1.1, o condensado que a princípio teria degenerescência entre os níveis $m_{f}=-1,0,1$ sendo o nível $f=1$ em confinamento, pode ter tal degenerescência quebrada na presença de um campo magnético, como é feito nos experimentos recentes [17], cujo valores do campo permitem uma descrição perturbativa.

Por completeza, passemos a descrição que aqui e nos trabalhos recentes é usada para descrever a parte hamiltoniana de partícula individual, com o campo eletromagnético tratado classicamente.

\subsubsection{Elementos de interação Átomo-Luz sob aproximação de Dipolo}

Para uma descrição coerente com a Mecânica Quântica, é necessário a partir da Lagrangiana de partículas carregadas obter a referente hamiltoniana, fornecendo segundo [27]

$$
\begin{aligned}
L[\mathbf{x}, \dot{\mathbf{x}}] & =\sum_{n}\left[\frac{m_{n}}{2} \dot{\mathbf{x}}_{n}^{2}(t)-q_{n} \phi\left(\mathbf{x}_{n}(t), t\right)+q_{n} \dot{\mathbf{x}}_{n}(t) \mathbf{A}\left(\mathbf{x}_{n}(t), t\right)\right] \\
H[\mathbf{x}, \mathbf{p}] & =\sum_{n}\left\{\frac{1}{2 m_{n}}\left[\mathbf{p}_{n}(t)-q_{n} \mathbf{A}\left(\mathbf{x}_{n}(t), t\right)\right]^{2}+q_{n} \phi\left(\mathbf{x}_{n}(t), t\right)\right\}, \\
& \operatorname{com} p_{n}^{(i)} \doteq \frac{\partial L}{\partial \dot{x}_{n}^{(i)}} \Rightarrow m_{n} \dot{\mathbf{x}}_{n}(t)=\mathbf{p}_{n}(t)-q_{n} \mathbf{A}\left(\mathbf{x}_{n}(t), t\right),
\end{aligned}
$$

onde aqui foi omitido por enquanto a interação entre as partes já que os campos atuam como potencial externo e objetiva-se no momento escrever a parte da hamiltoniana sem interação. É possível verificar que as equações clássicas de movimento produzidas por esta formulação estão de acordo com a resultante da força de Lorentz para segunda lei de Newton. 
Sob uma transformação de calibre/gauge nota-se, contudo, que a hamiltoniana não é invariante, muito embora possamos sempre associar a uma transformação unitária, a saber

$$
\begin{array}{r}
H \stackrel{\text { gauge }}{\longrightarrow} H^{\prime}=\sum_{n}\left\{\frac{1}{2 m_{n}}\left[\mathbf{p}_{n}-q_{n} \mathbf{A}\left(\mathbf{x}_{n}, t\right)-q_{n} \nabla \alpha\left(\mathbf{x}_{n}, t\right)\right]^{2}+\right. \\
\left.q_{n} \phi\left(\mathbf{x}_{n}, t\right)-q_{n} \frac{\mathrm{d} \alpha\left(\mathbf{x}_{n}, t\right)}{\mathrm{d} t}\right\} .
\end{array}
$$

Definindo convenientemente uma transformação unitária e em decorrência dela, utilizando $p_{n}^{(i)}$ denotando a i-ésima componente espacial do momento da n-ésima partícula, encontra-se

$$
\begin{aligned}
& U\left(\mathbf{x}_{1}, \ldots, \mathbf{x}_{n}, t\right) \doteq \exp \left[\frac{i}{\hbar} \sum_{n} q_{n} \alpha\left(\mathbf{x}_{n}, t\right)\right], \\
& {\left[p_{n}^{(i)}, U^{\dagger}\right]=-q_{n} \frac{\partial \alpha\left(\mathbf{x}_{n}, t\right)}{\partial x_{n}^{(i)}} U^{\dagger} \Rightarrow U \mathbf{p}_{n} U^{\dagger}=\mathbf{p}_{n}-q_{n} \nabla_{n} \alpha\left(\mathbf{x}_{n}, t\right),} \\
& i \hbar \frac{\mathrm{d} U}{\mathrm{~d} t} U^{\dagger}=-\sum_{n} q_{n} \frac{\partial \alpha\left(\mathbf{x}_{n}, t\right)}{\partial t},
\end{aligned}
$$

após fazer uso intensivo da propriedade $\left[p_{n}^{(i)}, f\left(\mathbf{x}_{m}\right)\right]=i \hbar \delta_{n m} \partial f / \partial x_{n}^{(i)}$. Com esse ferramental, e inserindo devidamente $\mathbb{1}=U U^{\dagger}$, pode-se sem muito empecilho concluir que, (2.69) é obtida por

$$
H^{\prime}=U H U^{\dagger}+i \hbar \frac{\mathrm{d} U}{\mathrm{~d} t} U^{\dagger} \mapsto i \hbar \frac{\mathrm{d} \Psi^{\prime}}{\mathrm{d} t}(\overrightarrow{\mathbf{x}}, t)=H^{\prime} \Psi^{\prime}(\overrightarrow{\mathbf{x}}, t) ; \Psi^{\prime}(\overrightarrow{\mathbf{x}}, t)=U \Psi(\overrightarrow{\mathbf{x}}, t)
$$

com a abreviação $\overrightarrow{\mathbf{x}} \equiv \mathbf{x}_{1}, \ldots, \mathbf{x}_{n}$.

Portanto a mudança de gauge é uma transformação unitária, o que mantém valores médios e amplitudes de transições inalterados. Em particular no dito gauge de Coulomb onde escolhe-se $\alpha(\mathbf{x}, t)$ de tal forma que $\nabla \cdot \mathbf{E}(\mathbf{x}, t)=\nabla \cdot \mathbf{A}(\mathbf{x}, t)=0$, onde a primeira resulta da ausência de fontes, que geram os campos.

Esta última asserção, nos garante $\nabla \phi(\mathbf{x}, t)=0$ ao verificar as transformações de Fourier que relaciona o campo aos potenciais $E=-\nabla \phi-\partial \mathbf{A} / \partial t$ e portanto excluir $\phi$ da descrição, por ser uma constante.

A aproximação de dipolo se dá quando o comprimento de onda da luz incidente é bem maior que a dimensão típica de raio atômico. Abrindo mão da generalidade até aqui, o conjunto de partículas pode ser considerado como os elétrons "ligados" do átomo. Neste caso nos interessa o campo na posição específica do átomo, a ser denotada por $\mathbf{R}$. 


$$
H=\sum_{n} \frac{1}{2 m_{e}}\left[\mathbf{p}_{n}-q \mathbf{A}(\mathbf{R}, t)\right]^{2},
$$

onde como mencionado anterior, foi excluído o potencial escalar $\phi$ no gauge de Coulomb.

Introduz-se então uma transformação de gauge cujo efeito seja

$$
T \mathbf{p}_{n} T^{\dagger}=\mathbf{p}_{n}+q_{n} \mathbf{A}(\mathbf{R}, t) \stackrel{(2.71)}{=} \mathbf{p}_{n}-q_{n} \nabla_{n} \alpha\left(\mathbf{x}_{n}, t\right)
$$

A solução analisando a segunda igualdade é trivial, dada por

$$
\alpha\left(\mathbf{x}_{n}, t\right)=-\mathbf{x}_{n} \cdot \mathbf{A}(\mathbf{R}, t)
$$

Com este gauge, de volta a (2.69) $\operatorname{com} \phi \equiv 0$ temos:

$$
H=\sum_{n} \frac{\mathbf{p}_{n}^{2}}{2 m_{e}}+\sum_{n}\left(q \mathbf{x}_{n}\right) \cdot \frac{\partial \mathbf{A}}{\partial t}=\sum_{n} \frac{\mathbf{p}_{n}^{2}}{2 m_{e}}-\mathbf{D} \cdot \mathbf{E}(\mathbf{R}, t)
$$

Conclui-se então, que nessas condições a interação com laser é dado simplesmente adicionando $H_{d i p}=-\mathbf{D} \cdot \mathbf{E}(\mathbf{R}, t)$ a hamiltoniana livre da onde: $\mathbf{D} \doteq \sum_{n} q \mathbf{x}_{n}$, é conhecido como operador de dipolo, que dá o nome para a aproximação.

Por fixação de que de fato isto é o que procuramos para este tema os feixes laser usados nos experimentos correntes são da ordem de $0.8 \mu \mathrm{m} \approx\left(10^{3} \sim 10^{4}\right) a_{o}$, confirmado em [17], sendo $a_{o}$ o "tamanho" típico dos átomos, alguns Angstrons.

\subsubsection{Transições Raman}

É imediado de (2.77) que podemos apenas estabelecer sentido no que tange transições já que o termo $H_{d i p}$ depende do tempo, se tratando de lasers. Entretanto vamos novamente em busca de um potencial efetivo em sua descrição, utilizando um arranjo que priorize ressonância, entre níveis específicos.

Evidentemente o átomo tem um espectro de estados excitados complexo para ser discutido aqui, então serão simplesmente enumerados, incluindo toda estrutura, inclusive os eventuais níveis hiperfinos. 
A expressão até segunda ordem das amplitudes de transição de $i$ para $m$ é

$$
\begin{aligned}
c_{m}^{(i)}(t)=\delta_{m i}-\frac{i}{\hbar} \int_{t_{o}}^{t} \mathrm{e}^{i \omega_{m i} t^{\prime}} V_{m i}\left(t^{\prime}\right) \mathrm{d} t^{\prime} & \\
& +\left(\frac{i}{\hbar}\right)^{2} \sum_{n} \int_{t_{o}}^{t} \mathrm{e}^{i \omega_{m n} t^{\prime}} V_{m n}\left(t^{\prime}\right) \mathrm{d} t^{\prime} \int_{t_{o}}^{t^{\prime}} \mathrm{e}^{i \omega_{n i} t^{\prime \prime}} V_{n i}\left(t^{\prime \prime}\right) \mathrm{d} t^{\prime \prime} .
\end{aligned}
$$

Por se tratar de átomos em um condensado, o estado inicial será tomado como o fundamental. $V(t)$ seguindo (2.77) pode ser expresso, através dos campos de cada laser $\mathbf{E}_{j}(\phi, t)=$ $\mathbf{E}_{o}^{j}\left(e^{i f_{j} t+i \phi_{j}}+e^{-i f_{j} t-i \phi_{j}}\right) / 2$, em seus elementos de matriz na base que compõem o espectro, como

$$
V_{k l}(t)=\sum_{j}-\hbar \Omega_{k l}^{(j)}\left(e^{i\left(f_{j} t+\phi_{j}\right)}+c . c\right) \quad \text { com } \quad \Omega_{k l}^{(j)} \doteq\langle k|\mathbf{D}| l\rangle \cdot \mathbf{E}_{o}^{j} / 2 \hbar .
$$

Por hora, será desenvolvido o formalismo para dois campos(lasers), ou seja com $j=1,2$, contudo é possível estender para acoplamentos usando mais campos. É importante observar que as fases $\phi_{j}$ dependem da posição atômica através do vetor de onda $\mathbf{k}_{j} \cdot \mathbf{R}$, embora não seja importante na descrição de transições dos níveis atômicos internos, será de suma importância quando tornarmos a analisar o conjunto de átomos.

Parte-se de uma hipótese fundamental, que deve ser alcançada no arranjo experimental, como já o foi em [17], onde $\left|f_{i}-\omega_{k 0}\right|, \omega_{k 0} \gg\left|\delta_{f}-\omega_{10}\right|$, onde os dois primeiros níveis enumerados correspondem a $\sigma=-1,0$, subníveis do primeiro estado hiperfino $f=1$ com a diferença de $\hbar \omega_{10}=E(\sigma=0)-E(\sigma=-1)$ e de frequência entre lasers $\delta_{f} \doteq\left|f_{1}-f_{2}\right|$.

O significado quantitativo de " $\gg$ " vem a depender da escolha específica das frequências dos lasers e, dependendo o arranjo pode acoplar os 3 níveis de $f=1$ como em $[18,28]$. As condições para acoplar apenas dois níveis serão ressaltadas a frente.

Dito em outras palavras, as transições esperadas pelo efeito individual de cada feixe está apreciavelmente longe da ressonância comparado com a defasagem entre suas frequências o estão com relação aos dois níveis mais baixos.

Nessas condições dois termos se pronunciam em (2.78), segundo as asserções anteriores, da parte correspondente a segunda ordem, já que em primeira ordem, volta-se a enfatizar aqui, estão longes da ressonância. 


$$
\begin{aligned}
c_{m}^{(i)}(t)=\delta_{m i} & +i^{2} \sum_{n}\left\{\Omega_{n i}^{(1)} \Omega_{m n}^{(2)}\left[\frac{e^{i \delta \phi}\left(e^{i\left(\omega_{m i}+\delta_{f}\right) t}-1\right)}{\left(\omega_{n i}+f_{1}\right)\left(\omega_{m i}+\delta_{f}\right)}+\frac{e^{-i \delta \phi}\left(e^{i\left(\omega_{m i}-\delta_{f}\right) t}-1\right)}{\left(\omega_{n i}-f_{1}\right)\left(\omega_{m i}-\delta_{f}\right)}\right]\right. \\
& +\Omega_{n i}^{(2)} \Omega_{m n}^{(1)}\left[\frac{e^{-i \delta \phi}\left(e^{i\left(\omega_{m i}-\delta_{f}\right) t}-1\right)}{\left(\omega_{n i}+f_{2}\right)\left(\omega_{m i}-\delta_{f}\right)}+\frac{e^{i \delta \phi}\left(e^{i\left(\omega_{m i}+\delta_{f}\right) t}-1\right)}{\left(\omega_{n i}-f_{2}\right)\left(\omega_{m i}+\delta_{f}\right)}\right] \\
& \left.+\mathcal{O}\left(\frac{1}{\left(\omega_{n i} \pm f_{j}\right)\left(\omega_{m i} \pm 2\left(f_{j}+f_{k}\right)\right)} ; \frac{1}{\left(\omega_{n i} \pm f_{j}\right)\left(\omega_{m i}\right)}\right)\right\},
\end{aligned}
$$

omitida a parte de primeira ordem que depende de $\left(\omega_{m i} \pm f_{1,2}\right)^{-1}$, onde fora definido $\delta \phi \doteq$ $\phi_{1}-\phi_{2}$ e sem perda de generalidade adotado $f_{1}>f_{2}$.

Agora com $i=0$, por análise dos denominadores os termos mais relevante se tornam, com $m=1$, os que dependem de $\omega_{10}-\delta_{f}$ e, analogamente para $i=1$ e $m=0$ aqueles que contém $\omega_{01}+\delta_{f} \equiv \delta_{f}-\omega_{10}$ no denominador. Todas estas condições, conduzem a um tratamento considerando apenas o subespaço $f=1$ que contém as probabilidades de transição relevantes, e portanto pode-se truncar a soma da equação integral, levando a

$$
\begin{aligned}
c_{m}^{(i)}(t)=\delta_{m i}-\frac{i}{\hbar} \int_{t_{o}}^{t} \sum_{n} \mathrm{e}^{i \omega_{m n} t^{\prime}} V_{m n}\left(t^{\prime}\right) c_{n}^{(i)}\left(t^{\prime}\right) \mathrm{d} t^{\prime} & \\
& \approx \delta_{m i}-\frac{i}{\hbar} \int_{t_{o}}^{t} \sum_{n=0}^{2} \mathrm{e}^{i \omega_{m n} t^{\prime}} V_{m n}\left(t^{\prime}\right) c_{n}^{(i)}\left(t^{\prime}\right) \mathrm{d} t^{\prime},
\end{aligned}
$$

onde especificamente teremos, recorrendo a (2.80) com a soma truncada e, usando simetria de paridade dos estados para excluir os elementos diagonais $\Omega_{i i}^{(1,2)}=0$, além de dispensar os termos por hipótese fora de ressonância, obtém-se

$$
\begin{aligned}
& c_{1}^{0}(t) \approx i^{2}\left(\frac{\Omega_{20}^{(1)} \Omega_{12}^{(2)}}{\omega_{20}-f_{1}}+\frac{\Omega_{20}^{(2)} \Omega_{12}^{(1)}}{\omega_{20}+f_{2}}\right) \int_{0}^{t} \mathrm{~d} t^{\prime} e^{i\left(\omega_{10}-\delta_{f}\right) t^{\prime}-i \delta \phi}, \\
& c_{0}^{1}(t) \approx i^{2}\left(\frac{\Omega_{21}^{(1)} \Omega_{02}^{(2)}}{\omega_{21}+f_{1}}+\frac{\Omega_{21}^{(2)} \Omega_{02}^{(1)}}{\omega_{21}-f_{2}}\right) \int_{0}^{t} \mathrm{~d} t^{\prime} e^{i\left(\omega_{01}+\delta_{f}\right) t^{\prime}+i \delta \phi},
\end{aligned}
$$

onde fora escrito a exponencial e parte do denominador de forma conveniente como uma integral. Dado que $\omega_{20}=\omega_{21}+\omega_{10}$ e $f_{1}-f_{2} \simeq \omega_{10} \Rightarrow \omega_{20}-f_{1} \simeq \omega_{12}-f_{2}$. Esta última nos leva a definir outro parâmetro que aparece nos trabalhos recentes dito "single foton detuning" $\Delta \doteq f_{1}-\omega_{20} \simeq f_{2}-\omega_{21}$, que aqui será usado como denominador comum na expressão acima.

Como efeito final, reduzimos um potencial a um efetivo que atua na variedade de $f=1$ que em linhas gerais é essencialmente o mesmo tratamento apresentado no artigo de revisão [29, 
seção 4.3] intitulado "Effective Atomic Ground State Hamiltonian".

Como $f_{1,2} \gg \omega_{k l}$ no espectro de transição no subespaço $f=1$, pode-se agrupar segundo identidade vetorial, voltando a utilizar o operador de dipolo $\mathbf{D}_{i j}$ em seus elementos de matriz, denotando por $\wedge$ o produto vetorial, na forma

$$
\begin{aligned}
& c_{1}^{o}(t) \approx \frac{i}{4 \Delta \hbar^{2}}\left(\mathbf{D}_{20} \wedge \mathbf{D}_{12}\right) \cdot\left(i \mathbf{E}_{2} \wedge \mathbf{E}_{1}\right) e^{-i \delta \phi} \int_{0}^{t} \mathrm{~d} t^{\prime} e^{i\left(\omega_{10}-\delta_{f}\right) t^{\prime}}, \\
& c_{o}^{1}(t) \approx \frac{i}{4 \Delta \hbar^{2}}\left(\mathbf{D}_{20} \wedge \mathbf{D}_{12}\right) \cdot\left(-i \mathbf{E}_{2} \wedge \mathbf{E}_{1}\right) e^{i \delta \phi} \int_{0}^{t} \mathrm{~d} t^{\prime} e^{i\left(\omega_{01}+\delta_{f}\right) t^{\prime}}
\end{aligned}
$$

Então pode-se reconhecer um potencial efetivo pela similaridade com a expressão em primeira ordem para amplitudes de transição, dado explicitamente por

$$
V_{\text {Raman }}(t) \equiv \frac{\hbar}{2 \Delta}\left(\begin{array}{cc}
0 & \Omega e^{-i \delta \phi} e^{-i \delta_{f} t} \\
\Omega^{*} e^{i \delta \phi} e^{i \delta_{f} t} & 0
\end{array}\right),\left|E_{1}\right\rangle=\left(\begin{array}{l}
1 \\
0
\end{array}\right),\left|E_{0}\right\rangle=\left(\begin{array}{l}
0 \\
1
\end{array}\right)
$$

$\operatorname{com} \Omega \doteq\left(\mathbf{D}_{20} \wedge \mathbf{D}_{12}\right) \cdot\left(i \mathbf{E}_{2} \wedge \mathbf{E}_{1}\right) / 2 \hbar^{2}$, bem semelhante à metodologia em [29], e o ordenamento de energia decrescente de cima para baixo em vetores coluna.

É útil lembrar que a enumeração dos níveis foi feita iniciando de 0 para facilidade de notação, porém, como são os dois primeiros níveis do estado hiperfino $f=1$ há uma correspondência $\left|E_{1}\right\rangle \leftrightarrow|\sigma=0\rangle$ e $\left|E_{0}\right\rangle \leftrightarrow|\sigma=-1\rangle$. Será então adota daqui em diante a notação baseada em $|\sigma\rangle$.

Importante ressaltar o papel fundamental dos níveis $|\sigma\rangle$ não estarem linearmente espaçados, caso contrário amplitudes de transição $\sigma=0 \longleftrightarrow \sigma=1$ seriam comparáveis as duas que temos interesse $(\sigma=0,-1)$.

Para assegurar a presença de dois níveis, $\omega_{21}-\delta_{f}$ deve ser múltiplo de pelo menos algumas unidades de $\omega_{10}-\delta_{f}$ (um fator 3 já produziria uma probabilidade de transição 9 vezes menor por exemplo), que por analise dos denominadores de (2.80) torna $\left|c_{2}^{(1)}\right|^{2}$ menos expressiva que $\left|c_{1}^{(0)}\right|^{2}$.

É fundamental então a consideração de efeito Zeeman até segunda ordem(quadrático) na separação dos níveis, já que $\omega_{21}=\omega_{Z}^{(1)}+\epsilon_{Z}$ e $\omega_{10}=\omega_{Z}^{(1)}-\epsilon_{Z} \approx \delta_{f}$, da onde

$$
\omega_{Z}^{(1)}+\epsilon_{Z}-\delta_{f}=\alpha\left|\omega_{Z}^{(1)}-\epsilon_{Z}-\delta_{f}\right|
$$

Portanto ao estabelecer um valor de tolerância para $\alpha$, fixa-se um limite para diferença 
de frequência dos lasers e consequentemente para $|\delta| \doteq\left|\left(\omega_{Z}^{(1)}-\epsilon_{Z}\right)-\delta_{f}\right|$. Adiante serão comparados valores de experimentos recentes no acoplamento de dois níveis.

Podemos então escrever uma Hamiltoniana para átomo individual, envolvendo os graus de liberdade $\sigma=-1,0$, como

$$
\hat{h}_{o}=\left(\frac{\mathbf{p}^{2}}{2 m}+V_{\text {trap }}\right) \otimes \mathbb{1}_{2}+\mathbb{1}_{\mathbf{x}} \otimes\left(E_{0}|-1\rangle\left\langle-1\left|+E_{1}\right| 0\right\rangle\langle 0|\right)+V_{\text {Raman }}(t),
$$

e além disso remover a dependência temporal com

$$
U(t) \doteq \mathbb{1}_{\mathbf{x}} \otimes e^{i \hat{\sigma}_{z}\left(\delta_{f} t+\gamma\right) / 2} ; \quad i \hbar \frac{d U}{d t} U^{\dagger}=-\frac{\hbar \delta_{f}}{2} \hat{\sigma}_{z}
$$

sendo mera conveniência $\Omega=|\Omega| e^{-i \gamma}$. Reescalonando os níveis de energia para $E_{0}=-\hbar \omega_{10} / 2$ e $E_{1}=\hbar \omega_{10} / 2$ temos

$$
\hat{h}_{o}=\left(\frac{\mathbf{p}^{2}}{2 m}+V_{\text {trap }}\right) \otimes \mathbb{1}_{2}+\mathbb{1}_{\mathbf{x}} \otimes \frac{\hbar\left(\omega_{10}-\delta_{f}\right)}{2} \hat{\sigma}_{z}+\frac{\hbar|\Omega|}{2 \Delta}\left(\begin{array}{cc}
0 & e^{-i\left(\phi_{1}-\phi_{2}\right)} \\
e^{i\left(\phi_{1}-\phi_{2}\right)} & 0
\end{array}\right)
$$

A diferença de fase na posição do átomo depende dos números de onda dos feixes e carrega uma dependência espacial. Um arranjo comum é considerar sentidos opostos de propagação, numa mesma direção, digamos $\hat{\mathbf{e}}_{x}$, de tal forma que: $\phi_{j}=\mathbf{k}_{j} \cdot \mathbf{R}= \pm k_{j} x \Rightarrow \phi_{1}-\phi_{2}=2 k x$ com $2 k \doteq\left(k_{1}+k_{2}\right)$. Fazendo ainda $\delta \doteq \omega_{10}-\delta_{f}$, obtém-se

$$
\hat{h}_{o}=\left(\frac{\mathbf{p}^{2}}{2 m}+V_{\text {trap }}\right) \otimes \mathbb{1}_{2}+\mathbb{1}_{\mathbf{x}} \otimes \frac{\hbar \delta}{2} \hat{\sigma}_{z}+\frac{\hbar|\Omega|}{2 \Delta}\left(\begin{array}{cc}
0 & e^{-i 2 k x} \\
e^{i 2 k x} & 0
\end{array}\right)
$$

O cuidado na notação de operadores aqui adotado, mostra explicitamente que o último termo não é um operador separável como produto e, daí origina-se um acoplamento entre movimento orbital e os graus de spin. Um identificação comum de notação é fazer a correspondência com $|0\rangle=|\uparrow\rangle \mathrm{e}|-1\rangle=|\downarrow\rangle$.

Uma outra forma usual de se expressar, é passar esse acoplamento para o operador de momento por meio de: $U(x) \doteq e^{i \hat{\sigma}_{z} k x}$, da qual pode-se mostrar que

$$
U(x) p_{x}^{2} U^{\dagger}(x)=p_{x}^{2}-2 p_{x}(k \hbar) \sigma_{z}+\hbar^{2} k^{2} \sigma_{z}^{2},
$$


que segundo um arranjo conveniente transforma-se em

$$
\hat{h}_{o}=\frac{\mathbf{p}_{\perp}^{2}}{2 m}+\frac{\left(p_{x}-\hbar k \sigma_{z}\right)^{2}}{2 m}+V_{\text {trap }}(\mathbf{x})+\frac{\hbar \delta}{2} \sigma_{z}+\frac{\hbar|\Omega|}{2 \Delta} \sigma_{x}
$$

Por completeza podemos escrever a EGP descrevendo a densidade espacial destes dois níveis acoplados, omitindo os argumentos de $\psi$, na forma

$$
\begin{gathered}
i \hbar \frac{\partial}{\partial t}\left(\begin{array}{c}
\psi_{\uparrow} \\
\psi_{\downarrow}
\end{array}\right)=\left(V_{\text {trap }}(\mathbf{x}) \mathbb{1}_{2}+\mathcal{G}\right)\left(\begin{array}{c}
\psi_{\uparrow} \\
\psi_{\downarrow}
\end{array}\right) ; \quad \gamma \doteq \frac{k \hbar}{m} \mathrm{e} \\
\mathcal{G}=\left(\begin{array}{cc}
\frac{\mathbf{p}^{2}}{2 m}-\gamma p_{x}+\frac{\hbar \delta}{2}+\sum_{\sigma=\uparrow \downarrow} g_{\uparrow \sigma}\left|\psi_{\sigma}\right|^{2} & \frac{\hbar|\Omega|}{2 \Delta} \\
\frac{\hbar|\Omega|}{2 \Delta} & \frac{\mathbf{p}^{2}}{2 m}+\gamma p_{x}-\frac{\hbar \delta}{2}+\sum_{\sigma=\downarrow \uparrow} g_{\downarrow \sigma}\left|\psi_{\sigma}\right|^{2}
\end{array}\right)
\end{gathered}
$$

Essa representação é útil, pois em alguns trabalhos(como este) são investigados solitons em meios uniformes (sem armadilha), onde $V=0$. Ainda deve ser dito que a transformação unitária que relaciona as duas formas de acoplamento spin-orbital pode ser comparada a uma transformação de gauge, da onde não é novidade mudar a hamiltoniana, mas mantém os valores dos observáveis.

Um outro meio de reproduzir estes resultados é partindo de uma interação efetiva, isolada em 3 níveis, em que $f_{1}$ e $f_{2}$ estão associados a transições dos dois níveis mais baixos a um mesmo nível $|e\rangle$. Este método é discutido em [30] e trata da mesma relação de parâmetros de interesse aqui.

\subsubsection{Escalas de energia e unidades}

De volta a analisar os parâmetros, para desenvolver uma compatibilidade com os trabalhos recentes, temos, por (2.87), modificando para relacionar com $|\delta|$ as formas

$$
\begin{gathered}
|\delta|=\frac{2 \epsilon_{Z}}{\alpha-1} \quad \text { se } \delta>0 \\
|\delta|=\frac{2 \epsilon_{Z}}{\alpha+1} \quad \text { se } \delta<0
\end{gathered}
$$

Valores adotados em [17] são $\hbar \epsilon_{Z} \approx 2 E_{L}$ onde $E_{L} \doteq \hbar^{2} k^{2} / 2 m, k=k_{x}$ onde os $k_{1}$ e $k_{2}$ tem mesmo módulo, embora no arranjo experimental tenham uma abertura angular, onde $k$ vem a depender das componentes dos vetores de onda.

Portanto com o valor de $\lambda=804.1 \mathrm{~nm}$ obtém-se, sendo $E_{L}$ a escala típica adota no artigo, 


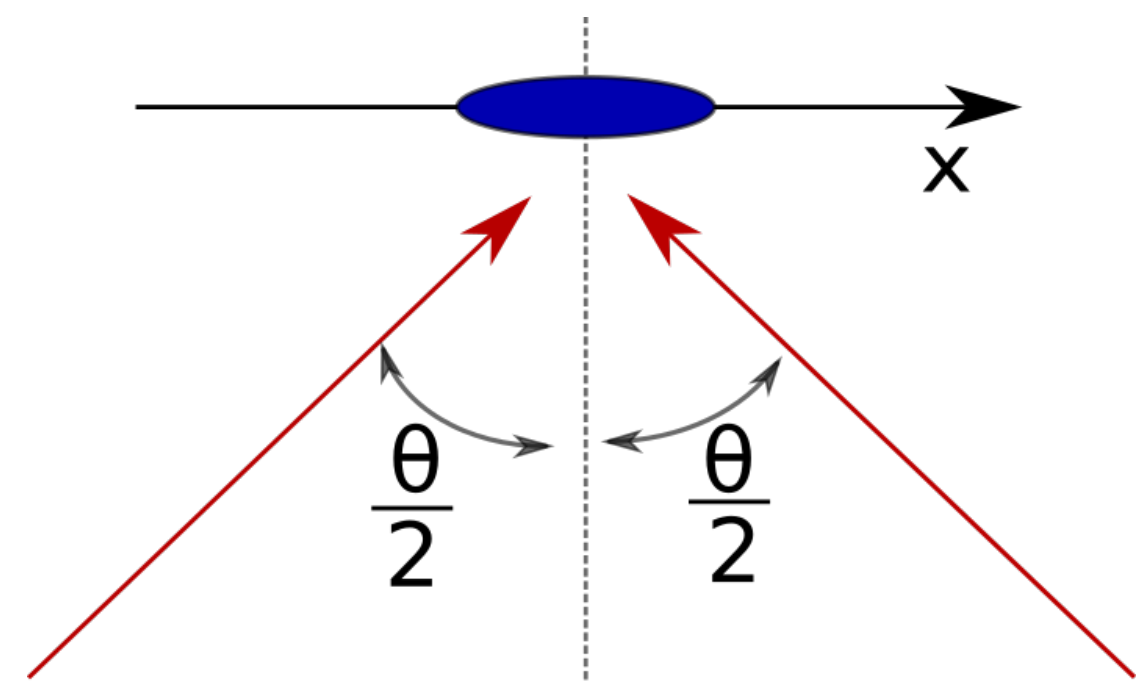

Figura 2.1: Arranjo experimental dos Lasers em [17], sendo utilizado $\theta=\pi / 2$, onde o acoplamento se dá na direção em que as componentes do vetor de onda estão em sentidos opostos.

$E_{L} / h \simeq 1.78 \mathrm{kHz}$. Isso fornece $\epsilon_{Z} / 2 \pi \simeq 3.56 \mathrm{kHz}$. Caso adotemos uma tolerância de $\alpha>5$ obtemos da segunda equação acima para $|\delta|$ a relação

$$
|\delta|<\frac{\epsilon_{Z}}{3} \quad \longrightarrow \quad|\delta| \lesssim 2 \pi \mathrm{kHz}
$$

Isso é de fundamental importância para que os casos estudados estejam dentro de condições experimentais possíveis.

Deixando de lado toda a generalidade considerada até então, este trabalho passa a restringirse no caso unidimensional, que contém muitos fenômenos de interesse estudados, já que o acoplamento com os lasers são ao longo de uma direção.

\section{Equações Unidimensionais}

No que tange um sistema unidimensional efetivo, é aquele onde o confinamento na direção transversal é muito mais forte que o longitudinal, assim $V_{\text {trap }}(x)=m \omega_{x}^{2} x^{2} / 2$ obedecendo $\omega_{x} \ll \omega_{\perp}$. As constantes de acoplamento mudam substancialmente, e se relacionam com o comprimento de espalhamento em 3 dimensões por: $g_{\sigma \sigma^{\prime}}^{(1 \mathrm{D})}=2 \hbar \omega_{\perp} a_{\sigma \sigma^{\prime}}$.

$\mathrm{Na}$ ausência de interações, a quantia $l_{\perp}=\sqrt{\hbar /\left(m \omega_{\perp}\right)}$ desempenha o papel do desvio padrão gaussiano na solução do oscilador harmônico quântico. Na idealização de um sistema unidimensional, seria zero e portanto deve fornecer uma escala de boa resolução para medida de comprimento.

Uma discussão análoga analisando o período das soluções estacionárias do oscilador harmônico 
leva a medir tempo em unidade de $\omega_{\perp}^{-1}$, bem como energia em $\hbar \omega_{\perp}$. Por conveniência introduzimos densidade medida em unidades de $\left(2\left|a_{\uparrow \uparrow}\right|\right)^{-1}$.

Em resumo, temos $x^{\prime} \doteq x / l_{\perp} \Rightarrow x \rightarrow x l_{\perp}$, de (2.94) $V_{\text {trap }} \rightarrow m \omega_{x}^{2} x^{2} l_{\perp}^{2} / 2,\left|\psi_{k}\right|^{2} \rightarrow$ $\left|\psi_{k}\right|^{2} /\left(2 a_{\uparrow \uparrow}\right), \partial_{x} \rightarrow \partial_{x} l_{\perp}^{-1}$ e $\partial_{t} \rightarrow \partial_{t} \omega_{\perp}$. Dividindo ambos lados da equação por $\hbar \omega_{\perp}$ por retratarem energia, obtemos

$$
\begin{aligned}
i \partial_{t}\left(\begin{array}{c}
\psi_{\uparrow} \\
\psi_{\downarrow}
\end{array}\right)= & {\left[\left(\frac{\omega_{x}^{2}}{\omega_{\perp}^{2}} x^{2}-\frac{\partial_{x}^{2}}{2}\right) \mathbb{1}_{2}+\left(i k l_{\perp} \partial_{x}+\frac{\delta}{2 \omega_{\perp}}\right) \hat{\sigma}_{z}+\right.} \\
& \left.\left(\begin{array}{cc} 
\pm\left|\psi_{\uparrow}\right|^{2}+\frac{a_{\uparrow \downarrow}}{\left|a_{\uparrow \uparrow}\right|}\left|\psi_{\downarrow}\right|^{2} & |\Omega| /\left(2 \Delta \omega_{\perp}\right) \\
|\Omega| /\left(2 \Delta \omega_{\perp}\right) & \frac{a_{\uparrow \downarrow}}{\left|a_{\uparrow \uparrow}\right|}\left|\psi_{\uparrow}\right|^{2}+\frac{a_{\downarrow \downarrow}}{\left|a_{\uparrow \uparrow}\right|}\left|\psi_{\downarrow}\right|^{2}
\end{array}\right)\right] \times\left(\begin{array}{c}
\psi_{\uparrow} \\
\psi_{\downarrow}
\end{array}\right) .
\end{aligned}
$$

O sinal $+(-)$ nos termos de interação, carregam informação desta ser repulsiva(atrativa). Deste ponto em diante, por conveniência na simplicidade da notação os parâmetros serão mudados para: $\Omega \rightarrow \Omega /\left(2 \Delta \omega_{\perp}\right), \delta \rightarrow \delta / 2 \omega_{\perp}, k_{L}=k l_{\perp}$, referente as interações $\beta \doteq a_{\uparrow \downarrow} /\left|a_{\uparrow \uparrow}\right|$ e $\gamma \doteq a_{\downarrow \downarrow} /\left|a_{\uparrow \uparrow}\right|$ e por fim $R=\omega_{x} / \omega_{\perp}$ para a razão entre as frequências da armadilha, encontra-se o par de equações acopladas não lineares

$$
\begin{aligned}
& i \partial_{t} \psi_{\uparrow}=\left(-\frac{\partial_{x}^{2}}{2}+i k_{L} \partial_{x}+R^{2} x^{2}+\delta \pm\left|\psi_{\uparrow}\right|^{2}+\beta\left|\psi_{\downarrow}\right|^{2}\right) \psi_{\uparrow}+\Omega \psi_{\downarrow}, \\
& i \partial_{t} \psi_{\downarrow}=\left(-\frac{\partial_{x}^{2}}{2}-i k_{L} \partial_{x}+R^{2} x^{2}-\delta+\beta\left|\psi_{\uparrow}\right|^{2}+\gamma\left|\psi_{\downarrow}\right|^{2}\right) \psi_{\downarrow}+\Omega \psi_{\uparrow} .
\end{aligned}
$$

Portanto todos os parâmetros passam a depender de $\omega_{\perp}$ que assume valores típicos entre $250 \sim 1000 \mathrm{~Hz}$. É útil salientar que temos então os novos valores possíveis experimentalmente, que passam a restringir $|\delta| \lesssim 3$. Claramente por também depender da intensidade de confinamento e, caso venha a se aprimorar os arranjos do campo magnético para aumentar as divisões de energia, podemos relaxar um pouco essa limitação em algumas unidades.

Sobre a normalização nesse sistema de unidade dada por $\int \mathrm{d} x \Psi^{\dagger} \Psi=\mathcal{N}$, se relaciona com o número de átomos, ou seja a norma antes da troca de unidade, por

$$
\mathcal{N}=\left(\frac{2\left|a_{\uparrow \uparrow}\right|}{l_{\perp}}\right) N
$$

Portanto a normalização carrega informação não apenas do número de átomos, mas também do confinamento do sistema nesta consideração unidimensional e da força de interação. Não 
menos importante, a energia média por átomo fica

$$
\begin{aligned}
\frac{\mathcal{E}}{N}=\frac{1}{\mathcal{N}} \int_{-\infty}^{\infty} \mathrm{d} x\left\{\Psi ^ { \dagger } \left[-\frac{\partial^{2}}{\partial x^{2}}+\right.\right. & \left.i k_{L} \hat{\sigma}_{z} \frac{\partial}{\partial x}+\delta \sigma_{z}+\Omega \sigma_{x}\right] \Psi \\
& \left.+\frac{1}{2}\left( \pm\left|\psi_{\uparrow}\right|^{4}+\gamma\left|\psi_{\downarrow}\right|^{4}\right)+\beta\left|\psi_{\uparrow}\right|^{2}\left|\psi_{\downarrow}\right|^{2}\right\} .
\end{aligned}
$$

Ao medir momento, nas unidades acima citadas temos $-i \partial / \partial x$ e, seu valor esperado será então denotado por $\langle k\rangle$. Pode-se introduzir o momento médio segunda uma espécie individual também, resumindo-se tudo em

$$
\langle k\rangle_{\lambda}=\frac{1}{\mathcal{N}} \int_{-\infty}^{\infty} \mathrm{d} x \psi_{\lambda}^{*}\left(-i \frac{\partial}{\partial x}\right) \psi_{\lambda}, \quad \lambda=\uparrow, \downarrow
$$

Por no momento dizer, a densidade de átomos pode também ser representada no espaço de momentos mediante a uma Transformada de Fourier, convencionada aqui como

$$
\psi_{\lambda}(x)=\int_{-\infty}^{\infty} \mathrm{d} k e^{i k x} \phi_{\lambda}(k), \quad \phi_{\lambda}(k)=\int_{-\infty}^{\infty} \mathrm{d} x e^{-i k x} \psi_{\lambda}(x)
$$

Por fim, eventualmente nos resultados, será levado em conta a polarização, correspondendo as matrizes de Pauli, $\hat{\sigma}_{l} \operatorname{com} l=x, y, z$, definidos por

$$
\left\langle\hat{\sigma}_{l}\right\rangle=\frac{1}{\mathcal{N}} \int_{-\infty}^{\infty} \mathrm{d} x \Psi^{\dagger} \hat{\sigma}_{l} \Psi, \quad \Psi=\left(\begin{array}{l}
\psi_{\uparrow} \\
\psi_{\downarrow}
\end{array}\right)
$$

Uma atenção especial deve ser dada para $\left\langle\hat{\sigma}_{z}\right\rangle$, que é um medidor de desbalanço de átomos entre os estados de spin, pois

$$
\left\langle\hat{\sigma}_{z}\right\rangle=\frac{\mathcal{N}_{\uparrow}-\mathcal{N}_{\downarrow}}{\mathcal{N}}, \quad \mathcal{N}_{\lambda}=\int_{-\infty}^{\infty} \mathrm{d} x\left|\psi_{\lambda}(x)\right|^{2}
$$

Estes observáveis, foram detalhados, pois serão a base de toda análise no capítulo seguinte. 


\section{Capítulo 3}

\section{Métodos Numéricos}

A equação a que se tem interesse (2.59), para os estudos nessa área, pela presença na não linearidade tornam escassas as soluções analíticas e, ao mesmo tempo é o que dá a riqueza de estudo para o tema. Por isso é recorrente a introdução de métodos numéricos para obtenção de solução.

Mesmo o tratamento numérico do problema tem seus contratempos e, pode ter um custo computacional alto, principalmente para sistemas em 3 dimensões. Portanto a busca constante por métodos numéricos mais rapidamente convergentes tem tomado boa parte da atenção para aqueles que estudam condensação.

Fica claro então que a compreensão dos resultados exigem um detalhamento dos métodos numéricos usados de forma a assegurar a credibilidade dos mesmos e, por vezes alguns casos onde é possível tratar analiticamente, a solução numérica pode ser feita para comparação.

No que segue duas classes principais são apresentadas, algoritmos de evolução temporal, que permitem a partir de uma condição inicial obter sua forma em qualquer instante posterior e, algoritmos para estados estacionários, os quais já assumem a dependência temporal explicita e iteram até a acurácia desejada.

\subsection{Algoritmos de Evolução Temporal}

Esta primeira classe de algoritmos pode ser usada em dois objetivos principais. Um de verificação, para testar as soluções obtidas nos métodos para estados estacionários e, assim averiguar se de fato o perfil de densidade não varia no tempo, aumentando a confiabilidade do resultado. Outro pela estabilidade de soluções, como os sólitons discutidos em capítulo anterior, observando se 
estes não se dispersam, que aqui será mostrado no capítulo seguinte.

Passemos então à descrição destes.

\subsubsection{Diferenças finitas, método Crank-Nicolson}

O problema básico é como abordar as derivadas, no caso em questão, para uma equação diferencial parcial do tipo de difusão (parabólica). Assim assumindo soluções contínuas e diferenciáveis (funções ditas "bem comportadas") temos em série de Taylor, para uma função genérica

$$
F(x+\delta x)=F(x)+\delta x \frac{\mathrm{d} F}{\mathrm{~d} x}+\frac{\delta x^{2}}{2} \frac{\mathrm{d}^{2} F}{\mathrm{~d} x^{2}}+\mathcal{O}\left(\delta x^{3}\right) .
$$

É imediato verificar que a expressão acima permite-nos expressar

$$
\begin{aligned}
\frac{\mathrm{d} F}{\mathrm{~d} x} & =[F(x+\delta x)-F(x-\delta x)] /(2 \delta x)+\mathcal{O}\left(\delta x^{3}\right) \\
\frac{\mathrm{d}^{2} F}{\mathrm{~d} x^{2}} & =[F(x+\delta x)-2 F(x)+F(x-\delta x)] / \delta x^{2}+\mathcal{O}\left(\delta x^{4}\right) .
\end{aligned}
$$

Fica claro das equações (2.94) que temos interesse na derivada primeira com respeito ao tempo e na segunda com respeito ao espaço. Ficam em aberto as condições de contorno que devem ser tomadas para que possamos calcular as derivadas espaciais no limiar do domínio da solução.

Aqui não se terá interesse nos extremos do domínio, pois serão tratados os casos de interação atrativa não armadilhados e, portanto deve-se tomar os limites do conjunto suficientemente longes do perfil inicial, onde a solução será nula em toda evolução temporal.

Com as aproximações acima, podemos transformar qualquer equação diferencial similar a de difusão em equação de diferenças. No entanto, as derivadas parciais espaciais, deveriam ser tomadas no instante $t+\delta t$ ou $t$ ? Essa questão leva a introdução de um parâmetro $\theta \in[0,1]$ tal que, dada uma equação genérica na forma

$$
\frac{\partial \Psi}{\partial t}=\Gamma\left(x, t, \Psi, \frac{\partial \Psi}{\partial x}, \ldots, \frac{\partial^{k} \Psi}{\partial x^{k}}\right)
$$

transformar-se em uma equação solúvel no contexto numérico como 


$$
\begin{array}{r}
{\left[\Psi_{m}^{n+1}-\Psi_{m}^{n}\right] / \delta t=\theta \Gamma\left(x_{m}, t_{n}, \Psi_{m}^{n},\left[\frac{\partial \Psi}{\partial x}\right]_{m}^{n}, \ldots,\left[\frac{\partial^{k} \Psi}{\partial x^{k}}\right]_{m}^{n}\right)+} \\
(1-\theta) \Gamma\left(x_{m}, t_{n+1}, \Psi_{m}^{n+1},\left[\frac{\partial \Psi}{\partial x}\right]_{m}^{n+1}, \ldots,\left[\frac{\partial^{k} \Psi}{\partial x^{k}}\right]_{m}^{n+1}\right) .
\end{array}
$$

$\mathrm{Na}$ expressão acima fora adotado a notação conveniente a forma computacional, onde os índices $m$ e $n$ se referem as quantias calculadas na posição $x_{m} \doteq L_{1}+m \delta x$ e no instante $t_{n} \doteq T_{o}+n \delta t$ respectivamente, com o domínio de solução fixado por $\mathcal{D}_{x}=\left[L_{1}, L_{2}\right]$ no espaço e $\mathcal{D}_{t}=\left[T_{o}, T^{\prime}\right]$ no tempo.

Embora não é foco do trabalho a generalidade de equações diferenciais parabólicas, temos que as ordens de derivadas espaciais que aparecem na equação calculadas no ponto $x_{m}$ dependerão dos valores da função numa certa vizinhança, que vem a englobar um conjunto de índices espaciais, digamos $m-l_{1}, \ldots, m+l_{2}$.

Para $\theta=1$ temos o que chamamos de método de diferenças explícito, pois fica imediato isolar $\Psi_{i}^{n+1}$ a partir do passo anterior $\Psi_{\left\{m+l_{1}, \ldots, m+l_{2}\right\}}^{n}$, começando de uma condição inicial arbitrária. Caso contrário temos o que chama-se método de diferenças implícito, pois temos de forma geral que resolver um sistema linear. O método Crank-Nicolson propriamente dito é a escolha $\theta=1 / 2[31]$.

Uma discussão heurística para a escolha do parâmetro $\theta$ é que pelo teorema do valor médio, sendo a solução uma função "bem comportada" as diferenças usadas para expressar a derivada temporal, vem a ser de fato a derivada num ponto intermediário entre $t_{n}$ e $t_{n+1}$. A escolha do método Crank-Nicolson não é por acaso, mas é pela estabilidade que oferece, diferente do método explicito que requer condições extras para a estabilidade da solução em como discretizar o espaço, explicitamente tratado em [32, 33].

\subsubsection{Métodos Espectrais}

Por vezes é proveitoso usar transformadas de Fourier para calcular derivadas, convencionadas aqui por

$$
\begin{aligned}
& f(t)=\mathcal{F}^{-1}[g](t)=\frac{1}{\sqrt{2}} \int \mathrm{d} \omega e^{i \omega t} g(\omega), \\
& g(\omega)=\mathcal{F}[f](\omega)=\frac{1}{\sqrt{2}} \int \mathrm{d} t e^{-i \omega t} f(t) .
\end{aligned}
$$


Temos portanto

$$
\begin{aligned}
\frac{\partial^{k} \Psi}{\partial u^{k}} & =\frac{1}{\sqrt{2}} \int \mathrm{d} \omega \frac{\partial^{k}}{\partial u^{k}} e^{i \omega u} \Phi(\omega) \\
& =\mathcal{F}^{-1}\left[(i \omega)^{k} \mathcal{F}[\Psi](\omega)\right](u) .
\end{aligned}
$$

De forma análoga este procedimento pode ser empregado para computar a exponencial de operadores que envolvam diferenciação, pois estes por definição são tratados como uma série de potências, logo

$$
\exp \left(\gamma \frac{\partial^{k}}{\partial u^{k}}\right) \Psi=\mathcal{F}^{-1}\left[e^{(i \omega)^{k} \gamma} \mathcal{F}[\Psi](\omega)\right](u) \quad \forall \gamma \in \mathbb{C}
$$

\subsubsection{Procedimento de separação de operadores}

Podemos por fim filtrar para o problema que nos propomos a resolver. Trata-se genericamente de hamiltonianas, que até então, ao menos em praticamente toda literatura conhecida, são separáveis em dois operadores,

$$
\mathcal{H}=\mathcal{K}\left(\frac{\partial}{\partial x}, \ldots, \frac{\partial^{k}}{\partial x^{k}}\right)+\mathcal{V}(x, \Psi)
$$

Para um sistema cuja evolução temporal é portanto

$$
i \frac{\partial \Psi}{\partial t}=\mathcal{H} \Psi
$$

a solução para um passo $\delta t$ pode ser apresentada, dada a a função $\Psi$ no instante $t_{o}$, como

$$
\Psi\left(x, t_{o}+\delta t\right)=\exp (-i(\mathcal{K}+\mathcal{V}) \delta t) \Psi\left(x, t_{o}\right)
$$

Como não se trata de números não poderíamos aplicar separadamente cada exponencial. No entanto, temos contexto aproximativo melhor, que aproxima em até segunda ordem nos operadores, dado em [34] por

$$
e^{\frac{\mathcal{B}}{2}} e^{\mathcal{A}} e^{\frac{\mathcal{B}}{2}}=\mathbb{1}+(\mathcal{A}+\mathcal{B})+\frac{1}{2}(\mathcal{A}+\mathcal{B})^{2}+\mathcal{O}\left(\mathcal{N}^{3}\right)
$$

onde $\mathcal{N}^{3}$ indica a ação três vezes de qualquer permutação de $\mathcal{A}$ e $\mathcal{B}$ e nos fornece a ordem do 
erro esperado. Uma rápida associação permite escrever então a solução

$$
\Psi\left(x, t_{o}+\delta t\right)=e^{-i \mathcal{V} \delta t / 2} e^{-i \mathcal{K} \delta t} e^{-i \mathcal{V} \delta t / 2} \Psi\left(x, t_{o}\right)
$$

Com isso podemos separar o problema em duas partes essenciais. A primeira é multiplicar por uma exponencial de números, que pode ou não envolver uma não linearidade e, resolver uma exponencial envolvendo operadores de diferenciação, onde pode-se escolher entre métodos espectrais ou Crank-Nicolson, discutidos acima.

\subsection{Algoritmos para soluções Estacionárias}

Antecipando o que será discutido, duas linhas principais de análise são aqui discutidas. A primeira se baseia numa rotação de Wick, tornando o tempo um parâmetro puramente imaginário, técnica usada para relacionar a equação de difusão de calor com a equação de Schrödinger. A segunda generalizando o método de Newton, passando de uma variável ordinária para um funcional e usando método interativo para solução de sistema linear.

\subsubsection{Propagação em Tempo imaginário - Relaxação}

De volta a (2.94), o operador $V_{\text {trap }}(\mathbf{x}) \mathbb{1}_{2}+\mathcal{G}$ possui um spectro, associado a seu conjunto de autovalores, i.e, dado $\Psi_{\mu} \in \mathcal{L}^{2}\left(\mathbb{R}^{3}, d x\right) \otimes \mathbb{C}^{2}$ temos

$$
\mu \Psi_{\mu}(\mathbf{x})=\left(V_{\text {trap }}(\mathbf{x}) \mathbb{1}_{2}+\mathcal{G}\right) \Psi_{\mu}(\mathbf{x})
$$

onde $\mu$ calha de ser o potencial químico(discussão na seção 2.3.1). Suponha então parte deste espectro enumerável e, que forme uma base para o espaço em questão. Portanto para todo $\boldsymbol{\Psi}(\mathrm{x}, t)$ que satisfaz: $i \hbar \partial_{t} \boldsymbol{\Psi}(\mathrm{x}, t)=\left(V_{\text {trap }}(\mathrm{x}) \mathbb{1}_{2}+\mathcal{G}\right) \Psi(\mathrm{x}, t)$ podemos expressá-lo como

$$
\boldsymbol{\Psi}(\mathbf{x}, t)=\sum_{\mu}\left\langle\Psi_{\mu}, \Psi(t=0)\right\rangle \Psi_{\mu}(\mathbf{x}) e^{-i \mu t / \hbar}
$$

com o produto interno usual $\langle\boldsymbol{\Phi}, \boldsymbol{\Lambda}\rangle \doteq \int \mathrm{d}^{3} \mathbf{x} \sum_{i}\left(\boldsymbol{\Phi}^{T}\right)_{i}^{*}(\mathbf{x}) \boldsymbol{\Lambda}_{i}(\mathbf{x})$. Introduzindo então $\tau \doteq i t$ e 
$\Phi(\mathrm{x}, \tau) \doteq \boldsymbol{\Psi}(\mathrm{x}, t)$, tratando posteriormente $\tau \in \mathbb{R}$, é imediato que

$$
\begin{aligned}
\hbar \partial_{\tau} \mathbf{\Phi}(\mathbf{x}, \tau) & =-\left(V_{\text {trap }}(\mathbf{x}) \mathbb{1}_{2}+\mathcal{G}\right) \mathbf{\Phi}(\mathbf{x}, \tau) \\
\boldsymbol{\Phi}(\mathbf{x}, \tau) & =\sum_{\mu}\left\langle\boldsymbol{\Psi}_{\mu}, \boldsymbol{\Psi}_{o}\right\rangle \boldsymbol{\Psi}_{\mu}(\mathbf{x}) e^{-\mu \tau / \hbar}
\end{aligned}
$$

Então assumindo que existe um único $\mu_{o}<\mu, \forall \mu$, a quantia

$$
\begin{aligned}
& \xi(\tau) \doteq \frac{\left\langle\boldsymbol{\Phi},\left(V_{\text {trap }}(\mathbf{x}) \mathbb{1}_{2}+\mathcal{G}\right) \boldsymbol{\Phi}\right\rangle}{\langle\boldsymbol{\Phi}, \boldsymbol{\Phi}\rangle}=\frac{\sum_{\mu}\left|\left\langle\boldsymbol{\Psi}_{\mu}, \boldsymbol{\Psi}_{o}\right\rangle\right|^{2} \mu e^{-2 \mu \tau / \hbar}}{\sum_{\mu}\left|\left\langle\boldsymbol{\Psi}_{\mu}, \boldsymbol{\Psi}_{o}\right\rangle\right|^{2} e^{-2 \mu \tau / \hbar}} \\
& =\frac{\mu_{o}+\sum_{\mu \neq \mu_{o}}\left|\frac{\left\langle\mathbf{\Psi}_{\mu}, \mathbf{\Psi}_{o}\right\rangle}{\left\langle\mathbf{\Psi}_{\mu_{o}}, \mathbf{\Psi}_{o}\right\rangle}\right|^{2} \mu e^{-2 \tau\left(\mu-\mu_{o}\right) / \hbar}}{1+\sum_{\mu \neq \mu_{o}}\left|\frac{\left\langle\mathbf{\Psi}_{\mu}, \mathbf{\Psi}_{o}\right\rangle}{\left\langle\mathbf{\Psi}_{\mu_{o}}, \mathbf{\Psi}_{o}\right\rangle}\right|^{2} e^{-2 \tau\left(\mu-\mu_{o}\right) / \hbar}} \stackrel{(\tau \rightarrow \infty)}{\longrightarrow} \mu_{o} .
\end{aligned}
$$

Com a mesma hipótese verifica-se, em meio a truques algébricos semelhantes, que

$$
\lim _{\tau \rightarrow \infty} \frac{\boldsymbol{\Phi}(\mathrm{x}, \tau)}{\langle\boldsymbol{\Phi}, \boldsymbol{\Phi}\rangle}=\frac{\left\langle\boldsymbol{\Psi}_{\mu_{o}}, \boldsymbol{\Psi}_{o}\right\rangle}{\left|\left\langle\boldsymbol{\Psi}_{\mu_{o}}, \boldsymbol{\Psi}_{o}\right\rangle\right|} \boldsymbol{\Psi}_{\mu_{o}}(\mathrm{x})
$$

sendo mais interessante expressá-lo como

$$
\left|\lim _{\tau \rightarrow \infty} \frac{\boldsymbol{\Phi}(\mathbf{x}, \tau)}{\langle\boldsymbol{\Phi}, \boldsymbol{\Phi}\rangle}\right|=\left|\Psi_{\mu_{o}}(\mathbf{x})\right|
$$

O método reside em evoluir temporalmente a equação (3.17) e, estabelecer certo critério de parada, obtendo ao fim, o estado com menor potencial químico associado.

Ocorre por vezes na expansão (3.16), $\left\langle\Psi_{\mu_{o}}, \Psi_{o}\right\rangle=0$ e assim o processo acima conduz, contudo, a $\Psi_{\mu_{1}}$ e, para se assegurar obter o estado "fundamental" pode-se aplicar o método uma segunda vez com uma condição inicial diferenciada.

\subsubsection{Estados Estacionários genéricos}

A abordagem aqui se baseia nos resultados comparativos de [35] em que o método que ficou conhecido como "método do Gradiente Conjugado" desempenhou o menor número de interações e, se destaca por sua notória simplicidade.

Seguindo o que foi obtido em (2.99) e (2.100), com $\Psi(x, t)=\left(\psi_{\uparrow} \psi_{\downarrow}\right)^{T}$, introduzimos soluções estacionárias como $\boldsymbol{\Psi}(x, t)=\boldsymbol{\Psi}(x) e^{-i \mu t}$. Pode-se ainda expressar introduzindo as 
partes reais e imaginárias $\psi_{\sigma}(x)=u_{\sigma}(x)+i v_{\sigma}(x), \operatorname{com} \sigma=\uparrow, \downarrow$

$$
\begin{aligned}
0 & =\left[\frac{-\partial_{x}^{2}}{2}+R^{2} x^{2}-\mu+\bar{\delta} \pm\left(u_{\uparrow}^{2}+v_{\uparrow}^{2}\right)+\beta\left(u_{\downarrow}^{2}+v_{\downarrow}^{2}\right)\right] u_{\uparrow}-k_{L} \partial_{x} v_{\uparrow}+\bar{\Omega} u_{\downarrow}, \\
0 & =\left[\frac{-\partial_{x}^{2}}{2}+R^{2} x^{2}-\mu+\bar{\delta} \pm\left(u_{\uparrow}^{2}+v_{\uparrow}^{2}\right)+\beta\left(u_{\downarrow}^{2}+v_{\downarrow}^{2}\right)\right] v_{\uparrow}+k_{L} \partial_{x} u_{\uparrow}+\bar{\Omega} v_{\downarrow}, \\
0 & =\left[\frac{-\partial_{x}^{2}}{2}+R^{2} x^{2}-\mu-\bar{\delta}+\beta\left(u_{\uparrow}^{2}+v_{\uparrow}^{2}\right)+\alpha\left(u_{\downarrow}^{2}+v_{\downarrow}^{2}\right)\right] u_{\downarrow}+k_{L} \partial_{x} v_{\downarrow}+\bar{\Omega} u_{\uparrow}, \\
0 & =\left[\frac{-\partial_{x}^{2}}{2}+R^{2} x^{2}-\mu-\bar{\delta}+\beta\left(u_{\uparrow}^{2}+v_{\uparrow}^{2}\right)+\alpha\left(u_{\downarrow}^{2}+v_{\downarrow}^{2}\right)\right] v_{\downarrow}-k_{L} \partial_{x} u_{\downarrow}+\bar{\Omega} v_{\uparrow} .
\end{aligned}
$$

O conjunto de equações acima pode ser representado como $\mathbf{L}_{o}[\mathbf{W}](x)=0$ com $\mathbf{W}(x) \doteq$ $\left(u_{\uparrow} v_{\uparrow} u_{\downarrow} v_{\downarrow}\right)^{T}$. Suponha então uma sequência convergente a solução, de tal forma que possamos escrever: $\mathbf{e}_{n}(\mathbf{x})=\mathbf{W}(x)-\mathbf{w}_{n}(x)$ e portanto $\mathbf{L}_{o}\left[\mathbf{w}_{n}\right](x)+\mathbf{L}_{o}\left[\mathbf{e}_{n}\right](x)=0$, podendo ser expresso convenientemente como

$$
\mathbf{L}_{o}\left[\mathbf{w}_{n}\right](x)+\mathbf{L}_{o}\left[\mathbf{W}-\mathbf{w}_{n}\right](x)=0 .
$$

Retomando o método de Newton para encontrar raízes de [36, cap. 9],

$$
f\left(x_{n}\right)+f^{\prime}\left(x_{n}\right)\left(x_{n+1}-x_{n}\right)=0
$$

Que para um conjunto de funções $f_{1}, \ldots, f_{m}$ de $l$ variáveis se generaliza para

$$
\begin{aligned}
& f_{j}\left(x_{1}^{(n)}, \ldots, x_{l}^{(n)}\right)+\sum_{k=1}^{l}\left(\partial_{x_{k}} f_{j}\right)\left(x_{k}^{(n+1)}-x_{k}^{(n)}\right)=0 ; \quad j=1, \ldots, m \\
\Rightarrow & \mathbf{f}\left(x_{1}^{(n)}, \ldots, x_{l}^{(n)}\right)+\mathcal{J} \cdot\left(x_{1}^{(n+1)}-x_{1}^{(n)} \ldots x_{l}^{(n+1)}-x_{1}^{(n+1)}\right)^{T}=0, \\
& \operatorname{com} \quad \mathcal{J}=\left(\begin{array}{ccc}
\partial_{x_{1}} f_{1} & \ldots & \partial_{x_{l}} f_{1} \\
\vdots & \ddots & \vdots \\
\partial_{x_{1}} f_{m} & \ldots & \partial_{x_{l}} f_{m}
\end{array}\right),
\end{aligned}
$$

com o índice superior indicando o processo interativo. Daí a relação traçada com o método de Newton usual, inferindo como devemos atualizar a sequência proposta, embora seja substancialmente diferente o conceito de derivada para o funcional $\mathbf{L}_{o}$.

O algoritmo consiste então em efetuar as trocas no segundo termo de (3.26), $\mathbf{L}_{o} \rightarrow \mathbf{L}_{1_{n}}$, cuja as derivadas devem ser computadas em $u_{\sigma}^{(n)}, v_{\sigma}^{(n)} \mathrm{e}, \mathbf{W} \rightarrow \mathbf{w}_{n+1}$ com a identificação $\mathbf{x}^{(n)} \rightarrow \mathbf{w}_{n}$. Agora o sentido mais próximo a $\mathbf{L}_{1_{n}}$ é utilizando derivadas funcionais (detalhado em [26, cap. 
33]) com relação as 4 funções, tratadas de forma independente, no conjunto de equações (3.22)(3.25) conduzindo a

$$
\mathbf{L}_{o}\left[\mathbf{w}_{n}\right](x)+\mathbf{L}_{1_{n}}\left[\Delta \mathbf{w}_{n}\right](x)=0 ; \quad \mathbf{w}_{n+1}=\mathbf{w}_{n}+\Delta \mathbf{w}_{n}
$$

onde o operador diferencial, dito "linearizado" $\mathbf{L}_{1}$, fica

$$
\begin{aligned}
& \mathbf{L}_{1}=\left(\begin{array}{cccc}
\mathcal{D}_{1} & -k_{L} \partial_{x} \pm 2 u_{\uparrow} v_{\uparrow} & \bar{\Omega}+2 \beta u_{\uparrow} u_{\downarrow} & 2 \beta u_{\uparrow} v_{\downarrow} \\
k_{L} \partial_{x} \pm 2 v_{\uparrow} u_{\uparrow} & \mathcal{D}_{2} & 2 \beta u_{\downarrow} v_{\uparrow} & \bar{\Omega}+2 \beta v_{\uparrow} v_{\downarrow} \\
\bar{\Omega}+2 \beta u_{\uparrow} u_{\downarrow} & 2 \beta u_{\downarrow} v_{\uparrow} & \mathcal{D}_{3} & k_{L} \partial_{x}+2 \alpha v_{\downarrow} u_{\downarrow} \\
2 \beta u_{\uparrow} v_{\downarrow} & \bar{\Omega}+2 \beta v_{\uparrow} v_{\downarrow} & -k_{L} \partial_{x}+2 \alpha u_{\downarrow} v_{\downarrow} & \mathcal{D}_{4}
\end{array}\right) \\
& \mathcal{D}_{1}=-\frac{\partial_{x}^{2}}{2}+R^{2} x^{2}-\mu+\bar{\delta} \pm\left(3 u_{\uparrow}^{2}+v_{\uparrow}^{2}\right)+\beta\left(u_{\downarrow}^{2}+v_{\downarrow}^{2}\right) \\
& \mathcal{D}_{2}=-\frac{\partial_{x}^{2}}{2}+R^{2} x^{2}-\mu+\bar{\delta} \pm\left(u_{\uparrow}^{2}+3 v_{\uparrow}^{2}\right)+\beta\left(u_{\downarrow}^{2}+v_{\downarrow}^{2}\right) \\
& \mathcal{D}_{3}=-\frac{\partial_{x}^{2}}{2}+R^{2} x^{2}-\mu-\bar{\delta}+\beta\left(u_{\uparrow}^{2}+v_{\uparrow}^{2}\right)+\alpha\left(3 u_{\downarrow}^{2}+v_{\downarrow}^{2}\right) \\
& \mathcal{D}_{4}=-\frac{\partial_{x}^{2}}{2}+R^{2} x^{2}-\mu-\bar{\delta}+\beta\left(u_{\uparrow}^{2}+v_{\uparrow}^{2}\right)+\alpha\left(u_{\downarrow}^{2}+3 v_{\downarrow}^{2}\right)
\end{aligned}
$$

Isso constitui as interação externas, de certa forma, uma extensão do método de Newton. Para encontrar então os $\Delta \mathbf{w}_{n}(\mathbf{x})$, é necessário resolver equações diferenciais ordinárias, que podem ser convertidas a sistemas lineares após discretização do espaço.

Para aplicar o método de Gradientes Conjugados, prosseguimos resolvendo um sistema linear após discretização do espaço, dado um erro inicial $\Delta \mathbf{w}_{o}(\mathbf{x})$, em geral nulo. é Importante frisar que para uma discretização espacial de $M$ pontos o sistema tem dimensão $4 M$.

\section{Método de Gradientes Conjugados}

A relação com gradiente provém da função definida a partir de uma matriz $\mathbb{A}$ e um vetor como

$$
g\left(x_{1}, \ldots, x_{n}\right)=\frac{1}{2} \mathbf{x}^{T} \cdot \mathbb{A} \cdot \mathbf{x}-\mathbf{b}^{T} \mathbf{x}
$$

cujo o gradiente em $n$ variáveis é

$$
\boldsymbol{\nabla} g=\frac{1}{2}\left(\mathbb{A}+\mathbb{A}^{T}\right) \cdot \mathbf{x}-\mathbf{b}=\mathbb{A} \cdot \mathbf{x}-\mathbf{b} ; \quad \text { se } \mathbb{A}=\mathbb{A}^{T}
$$

Em palavras a solução do sistema $\mathbb{A} \cdot \mathbf{x}=\mathbf{b}$ é um ponto de mínimo para $g$. Para o que segue, 
algumas definições importantes são listadas abaixo.

$$
\begin{aligned}
\mathbf{r}^{(k)} & =\mathbf{b}-\mathbb{A} \cdot \mathbf{x}^{(k)} \equiv-\nabla g \\
\mathbf{e}^{(k)} & =\mathbf{x}^{(k)}-\mathbf{x} \Rightarrow \mathbb{A} \cdot \mathbf{e}^{(k)}=-\mathbf{r}^{(k)} \\
\mathbf{x}^{(k+1)} & =\mathbf{x}^{(k)}+\alpha_{k} \mathbf{d}^{(k)}
\end{aligned}
$$

Seguindo a ideia de minimização impõe-se que o parâmetro $\alpha_{k}$ deve satisfazer

$$
\frac{\mathrm{d}}{\mathrm{d} \alpha_{k}} g\left(\mathbf{x}^{(k)}+\alpha_{k} \mathbf{d}\right)=\nabla g\left(\mathbf{x}^{(k+1)}\right) \cdot \frac{\mathrm{d} \mathbf{x}^{(k+1) T}}{\mathrm{~d} \alpha_{k}}=0,
$$

que conduz aos resultados, por (3.34) e (3.36),

$$
\begin{aligned}
& \mathbf{r}^{(k+1) T} \cdot \mathbf{d}^{(k)}=0, \\
& \alpha_{k}=\frac{\mathbf{d}^{(k) T} \cdot \mathbf{r}^{(k)}}{\mathbf{d}^{(k) T} \cdot \mathbb{A} \cdot \mathbf{d}^{(k)}} .
\end{aligned}
$$

Precisamos por fim definir este conjunto de direções $\mathbf{d}^{(k)}$. Para isto utilizaremos a ideia de que dois vetores são conjugados com relação a $\mathbb{A}$ se $\mathbf{u}^{T} \cdot \mathbb{A} \cdot \mathbf{v}=0$, e assim será imposto que

$$
\mathbf{d}^{(i) T} \cdot \mathbb{A} \cdot \mathbf{d}^{(j)}=0 ; \quad i \neq j
$$

Tomando um gerador para o espaço $\left\{\mathbf{u}_{o}, \ldots, \mathbf{u}_{n-1}\right\}$, e escolhendo $\mathbf{d}^{(o)}=\mathbf{u}_{o}$, escreve-se

$$
\mathbf{d}^{(i)}=\mathbf{u}_{i}+\sum_{k=0}^{i-1} \beta_{i k} \mathbf{d}^{(k)} ; \quad i \geq 1
$$

da onde (3.40) impõe

$$
\beta_{i j}=-\frac{\mathbf{u}_{i}^{T} \cdot \mathbb{A} \cdot \mathbf{d}^{(j)}}{\mathbf{d}^{(j) T} \cdot \mathbb{A} \cdot \mathbf{d}^{(j)}} ; \quad i>j
$$

Nota-se um conjunto de consequências dado (3.40) como válida, a saber das definições primordiais de erro e resíduo nas equações (3.34) a (3.36)

$$
\begin{aligned}
\mathbf{r}^{(k+1)} & =\mathbf{r}^{(k)}-\alpha_{k} \mathbb{A} \mathbf{d}^{(k)} \\
\mathbf{e}^{(k+1)} & =\mathbf{e}^{(k)}+\alpha_{k} \mathbf{d}^{(k)}
\end{aligned}
$$

Obtidas pelo simples uso de (3.36) em (3.34) e (3.35). Iterando as equações acima se obtém 
as relações

$$
\begin{aligned}
\mathbf{r}^{(k)} & =\mathbf{r}^{(o)}-\sum_{i=0}^{k-1} \alpha_{i} \mathbb{A} \mathbf{d}^{(i)} \\
\mathbf{e}^{(k)} & =\mathbf{e}^{(o)}+\sum_{i=0}^{k-1} \alpha_{i} \mathbf{d}^{(i)}
\end{aligned}
$$

Em vista de se usar a $\mathbb{A}$-conjugação das direções, obtemos respectivamente das equações acima $\mathbf{d}^{(j) T} \cdot \mathbb{A} \mathbf{e}^{(k)}=\mathbf{d}^{(j) T} \cdot \mathbb{A} \mathbf{e}^{(o)} \mathrm{e}, \mathbf{d}^{(j) T} \cdot \mathbf{r}^{(k)}=\mathbf{d}^{(j) T} \cdot \mathbf{r}^{(o)}$ caso $j \geq k$. Caso contrário, sendo $k>j$, obtemos

$$
\begin{aligned}
-\mathbf{d}^{(j) T} \mathbf{r}^{(k)}=\mathbf{d}^{(j) T} \cdot \mathbb{A} \mathbf{e}^{(k)} & =\mathbf{d}^{(j) T} \cdot \mathbb{A} \mathbf{e}^{(o)}+\alpha_{j} \mathbf{d}^{(j) T} \mathbb{A} \mathbf{d}^{(j)} \\
& =-\mathbf{d}^{(j) T} \cdot \mathbf{r}^{(o)}+\mathbf{d}^{(j) T} \cdot \mathbf{r}^{(j)} \\
& =0 .
\end{aligned}
$$

resultado alcançado pelo uso de (3.39) e a segunda igualdade de (3.35). Então, verifica-se que

$$
\begin{aligned}
\mathbf{d}^{(j) T} \mathbf{r}^{(k)} & =\mathbf{u}_{j}^{T} \mathbf{r}^{(k)}=0 ; \quad k>j, \\
\mathbf{d}^{(k) T} \mathbf{r}^{(k)} & =\mathbf{u}_{k}^{T} \mathbf{r}^{(k)}
\end{aligned}
$$

Até então não foi decidido o conjunto $\left\{\mathbf{u}_{o}, \ldots, \mathbf{u}_{n-1}\right\}$ e, escolhendo este como $\left\{\mathbf{r}^{(o)}, \ldots, \mathbf{r}^{(n-1)}\right\}$ é imediato que este seja linearmente independente(L.I) já que o algoritmo induz a ortogonalidade pela equação acima $\mathbf{r}_{o} \perp \mathbf{r}_{1} \perp \ldots \perp \mathbf{r}_{n-1}$.

Com esta escolha, (3.39) fica

$$
\alpha_{k}=\frac{\left\|\mathbf{r}^{(k)}\right\|^{2}}{\mathbf{d}^{(k) T} \cdot \mathbb{A} \cdot \mathbf{d}^{(k)}}
$$

O trunfo deste algoritmo se dá ao retornar e computar $\beta_{i j}$, pois

$$
\mathbf{r}^{(i) T} \mathbf{r}^{(j+1)}=\mathbf{r}^{(i) T} \mathbf{r}^{(j)}-\alpha_{j} \mathbf{r}^{(i) T} \mathbb{A} \mathbf{d}^{(j)}
$$

obtida pelo uso de (3.43). O lado direito tem o termo de interesse para o numerador de (3.42), que pelo fato de $j<i$ neste caso, só há um coeficiente não nulo, escolhendo $j=i-1$ acima e 
obtendo

$$
\beta_{i(i-1)}=\frac{1}{\alpha_{i-1}} \frac{\left\|\mathbf{r}^{(i)}\right\|^{2}}{\mathbf{d}^{(i-1) T} \cdot \mathbb{A} \cdot \mathbf{d}^{(i-1)}},
$$

que pela (3.50) se simplifica ainda para

$$
\beta_{i(i-1)}=\frac{\left\|\mathbf{r}^{(i)}\right\|^{2}}{\left\|\mathbf{r}^{(i-1)}\right\|^{2}} \longrightarrow \beta_{i} ; \quad i \geq 1
$$

O algoritmo em pseudo-código, reunindo tudo que foi dito fica

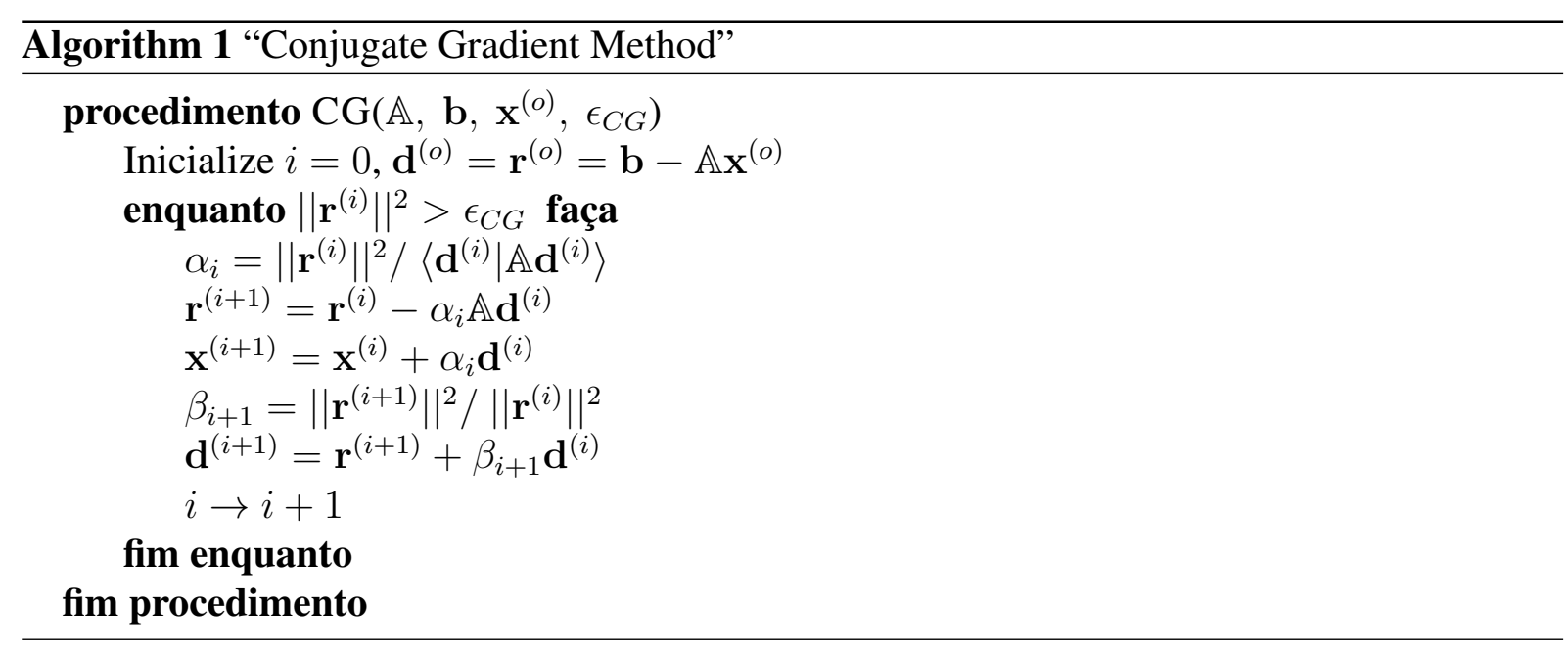

O processo termina com a solução exata após $\operatorname{Dim}(\mathbb{A})=n$ iterações caso a matriz envolvida seja positiva definida [37], o que se traduz em $\mathbf{v}^{T} \mathbb{A} \mathbf{v}>0, \forall \mathbf{v} \neq \mathbf{0}$. Mesmo sem esse requisito o algoritmo converge a solução, no entanto pode ocorrer a "falha" caso encontre em alguma das direções $\mathbf{d}^{T} \mathbb{A} \mathbf{d}=0$, podendo contudo recomeçar o processo com outra tentativa inicial $\mathrm{x}^{(o)}$.

A definição do erro residual $\epsilon_{C G}$ pode ser feita de acordo com o erro na interação de "Newton", para evitar uma acurácia muito maior do que necessário, processo nomeado de "oversolving" em [35].

Não deve passar despercebido a relação com os espaço de "Krylov" discutidos em [38, cap. 7] e [39, cap. 6]. Podemos do algoritmo acima ver que

$$
\begin{aligned}
\mathbf{d}^{(i+1)} & =\mathbf{r}^{(i)}-\alpha_{i} \mathbb{A} \mathbf{d}^{(i)}+\beta_{i+1} \mathbf{d}^{(i)} \\
& =\mathbf{r}^{(i)}-\alpha_{i} \mathbb{A}\left(\mathbf{r}^{(i)}+\beta_{i} \mathbf{d}^{(i-1)}\right)+\beta_{i+1}\left(\mathbf{r}^{(i)}+\beta_{i} \mathbf{d}^{(i-1)}\right) .
\end{aligned}
$$

Da onde, pode-se concluir deste procedimento que para cada $i$-ésima interação que se reduz na relação com o resíduo, adiciona-se uma multiplicação matricial por $\mathbb{A}$. Assim, se conclui que 
pode-se expressar

$$
\mathbf{d}^{(i)}=P_{i}(\mathbb{A}) \mathbf{r}^{(o)}
$$

embora não seja de fundamental interesse a forma explícita do polinômio $P_{i}$. Analogamente os resíduos, em qualquer estágio da iteração, podem ser também obtidos por um polinômio atuando no primeiro resíduo $\mathbf{r}^{(o)}$, conduzindo a

$$
\mathbf{d}^{(i)}, \mathbf{r}^{(i)} \in \operatorname{Ger}\left(\left\{\mathbf{r}^{(o)}, \mathbb{A} \mathbf{r}^{(o)}, \ldots, \mathbb{A}^{i} \mathbf{r}^{(o)}\right\}\right) \equiv \mathcal{K}_{i}\left(\mathbb{A}, \mathbf{r}^{(o)}\right)
$$

O espaço gerado acima, recebe o nome de "Espaço de Krylov". Mais do que isso, de volta a analisar (3.48), temos ainda

$$
\mathbf{r}^{(k)} \perp \mathcal{K}_{i}\left(\mathbb{A}, \mathbf{r}^{(o)}\right) \quad \text { se } k>i
$$

Essa última, escrita em termos do gradiente, junto com a (3.48) trocado pela escolha: $\mathbf{r}^{(k)}=$ $\mathbf{u}_{k}$, formam os pilares dos métodos interativos variantes para matrizes não simétricas e de forma mais explícita:

$$
\mathbf{r}^{(i)}, \mathbf{d}^{(i)} \perp \nabla g\left(\mathbf{x}^{(k)}\right) \quad \text { se } k>i
$$

\section{Método de Gradientes Bi-Conjugados e Estabilização}

Como se deve notar de (3.31), o termo envolvendo uma derivada, é responsável pela não simetria do operador, e não há um correspondente matricial, após discretização espacial, cujo $\mathbb{A}=\mathbb{A}^{T}$. Com o auxílio das seguintes definições, por meio de especificação em blocos

$$
\underbrace{\left(\begin{array}{cc}
\mathbb{A} & 0 \\
0 & \mathbb{A}^{T}
\end{array}\right)}_{\mathbb{B}} \underbrace{\left(\begin{array}{l}
\mathbf{x} \\
\mathbf{x}^{\prime}
\end{array}\right)}_{\mathbf{y}}=\underbrace{\left(\begin{array}{l}
\mathbf{b} \\
\mathbf{b}
\end{array}\right)}_{\mathbf{c}}, \mathbb{H} \doteq\left(\begin{array}{cc}
0 & \mathbb{A}^{T} \\
\mathbb{A} & 0
\end{array}\right) \text { e } \mathbb{J} \doteq\left(\begin{array}{ll}
0 & \mathbb{1} \\
\mathbb{1} & 0
\end{array}\right) \Rightarrow \mathbb{J} \cdot \mathbb{B}=\mathbb{H},
$$

da onde é imediato que: $\mathbb{H}=\mathbb{H}^{T}, \mathbb{J}=\mathbb{J}^{T}$ e $\mathbb{B} \neq \mathbb{B}^{T}$. Com a definição: $g_{H}(\mathbf{x})=\frac{1}{2}(\mathbf{a}-$ $\mathbf{x})^{T} \mathbb{H}(\mathbf{a}-\mathbf{x})$, para algum “a” fixo, pré definido. Tal função produz, para a escolha: $\mathbf{a}=\mathbb{B}^{-1} \mathbf{c}$ :

$$
\nabla g_{H}(\mathbf{x})=\mathbb{H} \cdot\left(\mathbf{x}-\mathbb{B}^{-1} \mathbf{c}\right)=\mathbb{J} \cdot \mathbb{B} \cdot \mathbf{x}-\mathbb{J} \mathbf{c}
$$

O que deve se anular para $\mathbf{x}=\mathbf{y}$, ou seja, na solução. Prossegue-se então de maneira 
totalmente similar a anterior, com a lista de relações:

$$
\begin{aligned}
\mathbf{s}^{(k)} & =\mathbf{c}-\mathbb{B} \mathbf{y}^{(k)} \\
\mathbf{q}^{(k)} & =\mathbf{s}^{(k)}+\sum_{i=0}^{k-1} \beta_{k, i} \mathbf{q}^{(i)} \\
\mathbf{y}^{(k+1)} & =\mathbf{y}^{(k)}+\alpha_{k} \mathbf{q}^{(k)} \\
\mathbf{s}^{(k+1)} & =\mathbf{s}^{(k)}-\alpha_{k} \mathbb{B} \mathbf{q}^{(k)} \\
\nabla g_{H}\left(\mathbf{y}^{(k)}\right) & =-\mathbb{J} \cdot \mathbf{s}^{(k)}
\end{aligned}
$$

Com as duas últimas consequências das três primeiras. A (3.58) para este caso mostra

$$
\begin{aligned}
\beta_{k, k-1} & =\frac{\left\langle\mathbf{s}^{(k)} \mid \mathbb{J} \mathbf{S}^{(k)}\right\rangle}{\left\langle\mathbf{s}^{(k-1)} \mid \mathbb{J} \mathbf{S}^{(k-1)}\right\rangle} \rightarrow \beta_{k} \\
\alpha_{k} & =\frac{\left\langle\mathbf{s}^{(k)} \mid \mathbb{J} \mathbf{S}^{(k)}\right\rangle}{\left\langle\mathbf{q}^{(k)} \mid \mathbb{H} \mathbf{q}^{(k)}\right\rangle}
\end{aligned}
$$

Após uso das séries de propriedades acima listadas. Por se tratarem de matrizes expressas em blocos, os números são mais convenientemente expressos como

$$
\begin{aligned}
\left\langle\mathbf{s}^{(k)} \mid \mathbb{J} \mathbf{s}^{(k)}\right\rangle & =\left(\begin{array}{c}
\mathbf{r}^{(k)} \\
\mathbf{r}^{\prime(k)}
\end{array}\right)\left(\begin{array}{ll}
0 & \mathbb{1} \\
\mathbb{1} & 0
\end{array}\right)\left(\begin{array}{l}
\mathbf{r}^{(k)} \\
\mathbf{r}^{\prime(k)}
\end{array}\right)=2\left\langle\mathbf{r}^{\prime(k)} \mid \mathbf{r}^{(k)}\right\rangle \\
\left\langle\mathbf{q}^{(k)} \mid \mathbb{H} \mathbf{q}^{(k)}\right\rangle & =\left(\begin{array}{l}
\mathbf{d}^{(k)} \\
\mathbf{d}^{\prime(k)}
\end{array}\right)\left(\begin{array}{cc}
0 & \mathbb{A}^{T} \\
\mathbb{A} & 0
\end{array}\right)\left(\begin{array}{l}
\mathbf{d}^{(k)} \\
\mathbf{d}^{\prime(k)}
\end{array}\right)=2\left\langle\mathbf{d}^{\prime(k)} \mid \mathbb{A} \mathbf{d}^{(k)}\right\rangle
\end{aligned}
$$

Portanto, produz dois vetores a serem iterados, daí o nome "Bi-Conjugado", tomando a forma em pseudo-código, mostrada abaixo

É de fácil verificação que a rotina do algoritmo 2 necessita o dobro de recursos computacionais, em memória e processamento, frente a do 1. Quando se torna a analisar a convergência, como feita em [40, 41], encontra-se uma convergência irregular para o método Biconjugado que pode ser suavizada com a introdução de novos polinômios, atuando sobre o resíduo, processo nomeado estabilização.

Não está aqui em questão discutir a generalidade dos métodos, embora, um bom conhecimento evidencie a escolha prezando pela usabilidade com menor complexidade de implementação.

Seguindo a discussão de [42] e pelo problema encontrado em [40] no exemplo 1, a estabilização feita com polinômio de primeiro grau ("BiCG-stabilized(1)") pode estagnar o processo, onde 


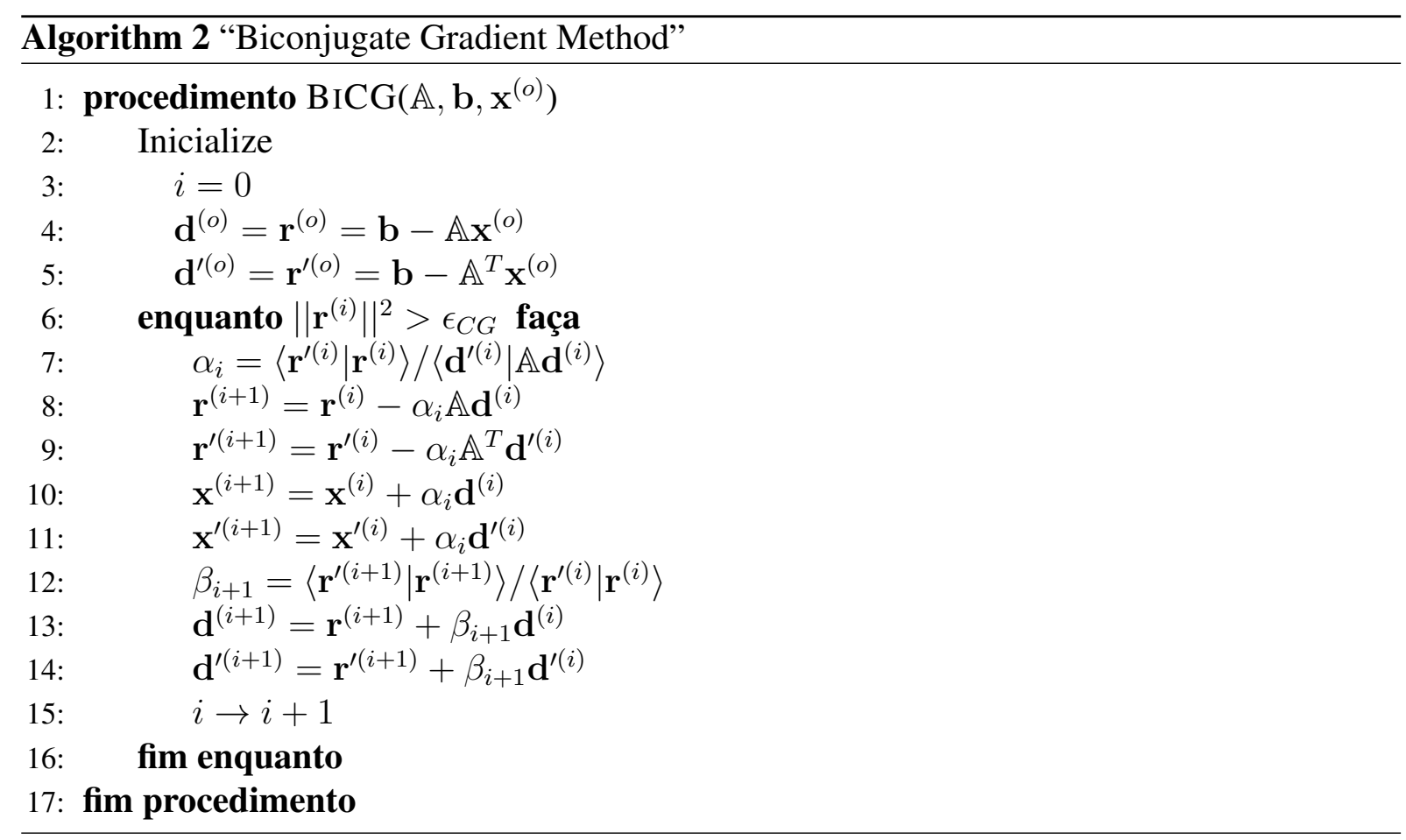

até mesmo a rotina 2 pode convergir e esta última não, devido a autovalores complexos. Mesmo sem informações sobre o espectro de $\mathbf{L}_{1_{n}}$ em (3.31), poderia ser uma trabalho em vão implementar com estabilização de primeiro grau.

A estratégia que será empregada aqui é em primeira estância usar o algoritmo 2 e, ao encontrar problemas de convergências partir para estabilização de segundo grau ("BiCGstab(2)" explicitado em [42]). 


\section{Capítulo 4}

\section{Resultados e Estudos Dirigidos}

A introdução da presente dissertação, ressaltou alguns dos diversos temas sendo estudados atualmente, referentes aos condensados cuja a dinâmica orbital está associada ao spin. Sendo este trabalho endereçado a resultados teóricos(analíticos e computacionais), a coleção de estudos específicos foi selecionada com base em artigos de relevância publicados recentemente já mencionados no decorrer do texto, reproduzindo e eventualmente estendendo seus conteúdos e resultados.

No que segue tudo se refere ao caso unidimensional, salvo menção explícita caso contrário.

\subsection{Autoestados sem interação}

Primeiramente volta-se a atenção para o espectro de (2.93) na forma unidimensional, que é a expressão entre colchetes em (2.102). Em um sistema uniforme sem armadilha, busca-se então por $\hat{h}_{o}|\mathcal{E}\rangle=\mathcal{E}|\mathcal{E}\rangle$. Sem perda de generalidade pode-se propor

$$
\langle x \mid \mathcal{E}\rangle=\left(\begin{array}{c}
\chi_{\uparrow} \\
\chi_{\downarrow}
\end{array}\right) e^{i k x},
$$

sendo que o sistema a que se chega,

$$
\left\{\begin{array}{c}
\left(\frac{k^{2}+k_{L}^{2}}{2}-k_{L} k+\delta-\mathcal{E}\right) \chi_{\uparrow}+\Omega \chi_{\downarrow}=0, \\
\Omega \chi_{\uparrow}+\left(\frac{k^{2}+k_{L}^{2}}{2}+k_{L} k-\delta-\mathcal{E}\right) \chi_{\downarrow}=0
\end{array}\right.
$$


deve ter solução não trivial e portanto (desprezando a constante $k_{L}^{2}$ )

$$
\mathcal{E}_{ \pm}=\frac{k^{2}}{2} \pm \sqrt{\left(k_{L} k-\delta\right)^{2}+\Omega^{2}}
$$

O estado fundamental reside no ramo definido por $\mathcal{E}_{-} \equiv \mathcal{E}$ e, os dois ramos coincidem no desligamento de todos os parâmetros $\Omega, k_{L}$ e $\delta$. Em seguida minimizando com relação o parâmetro livre $k$, denotando os pontos críticos de $\mathcal{E}$ por $k^{\prime}$, obtém-se

$$
\begin{aligned}
k^{\prime}-\frac{\left(k_{L} k^{\prime}-\delta\right) k_{L}}{\sqrt{\left(k_{L} k^{\prime}-\delta\right)^{2}+\Omega^{2}}} & =0, \\
k^{2}\left[\left(k_{L} k^{\prime}-\delta\right)^{2}+\Omega^{2}\right] & =\left(k_{L} k^{\prime}-\delta\right)^{2} k_{L}^{2}, \\
k^{\prime 2}\left[\left(k_{L} k^{\prime}-\delta\right)^{2}\right] & \leq\left(k_{L} k^{\prime}-\delta\right)^{2} k_{L}^{2}, \\
\left|k^{\prime}\right| & \leq\left|k_{L}\right| \text { se }\left(k_{L} k^{\prime}-\delta\right) \neq 0 .
\end{aligned}
$$

A igualdade é válida caso $\Omega=0$ como pode ser facilmente notado. Pode-se estudar brevemente dois casos analíticos, mas antes vejamos na figura a seguir como se comporta o gráfico $\mathcal{E} \times k$.

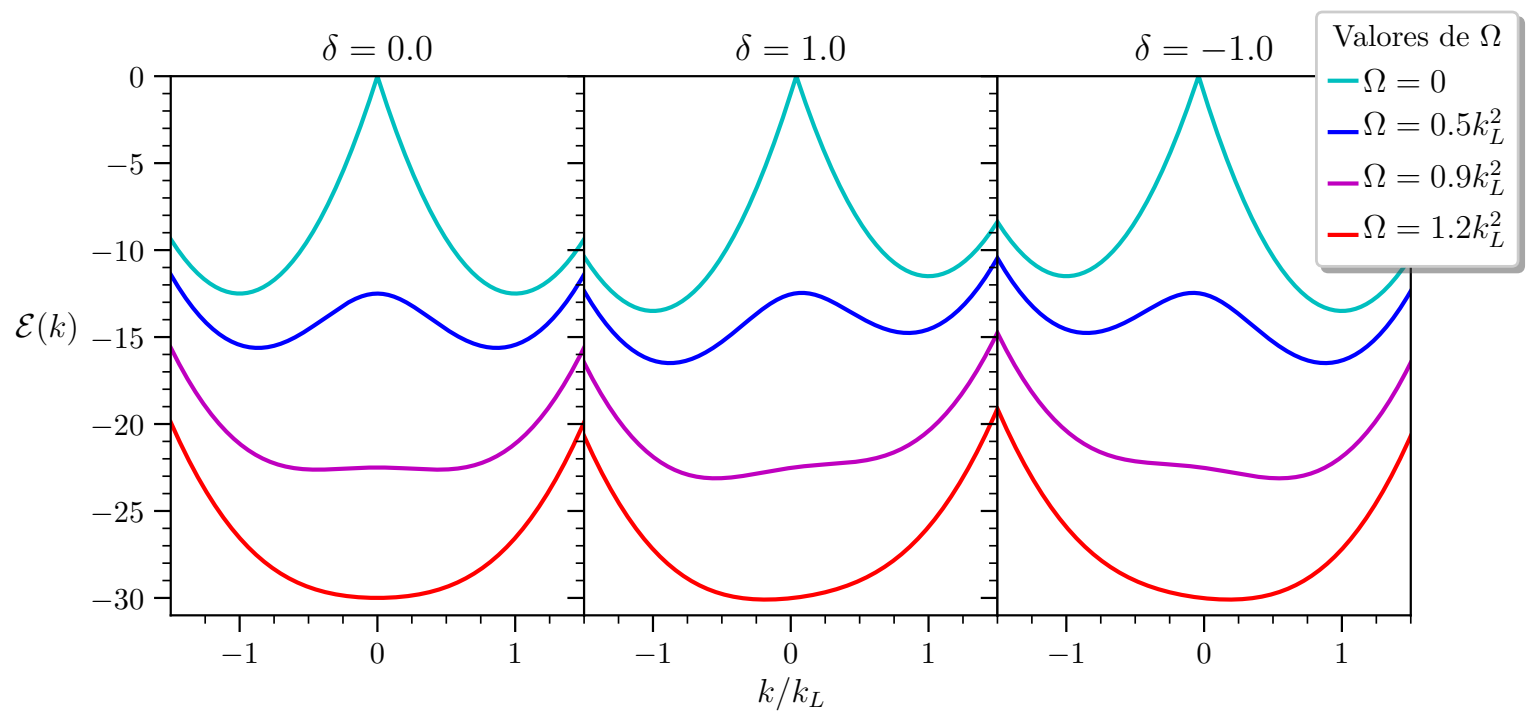

Figura 4.1: Ramo $\mathcal{E}_{-}$do espectro após diagonalização da Hamiltoniana na ausência de interações, para o valor fixo de $k_{L}=5.0$, deslocado do termo constante $k_{L}^{2} / 2$. Diferentes valores de $\delta$ e $\Omega$ mostram a existência de um ou dois mínimos e, se são locais ou globais.

Qualitativamente a figura acima é marcada por apresentar dois mínimos para valores baixos de $\Omega$ e $\delta$ atua tornando um desses mínimos global no domínio apresentado. Para grandes valores de $\Omega$ encontra-se apenas um mínimo em $k^{\prime}=0$, embora para $\delta \neq 0$ esse mínimo ocorra próximo 
mas não em zero, como a figura revela.

De fato da eq. (4.4), considerando $\Omega \gg\left|\left(k_{L} k-\delta\right)\right|$ e ignorando termos de $\mathcal{O}\left(\left(k_{L} k-\right.\right.$ $\left.\delta)^{2} / \Omega^{2}\right)$ encontra-se a simples expressão

$$
k^{\prime}=-\frac{\delta k_{L}}{\Omega\left(1-\frac{k_{L}^{2}}{\Omega}\right)} \quad \text { se } \quad \Omega \gg\left|\left(k_{L} k-\delta\right)\right|,
$$

que mostra a diferença do fator $\delta$ mesmo para valores altos de $\Omega$, deslocando o valor mínimo da energia de $k^{\prime}=0$.

Caso $\delta=\mathbf{0}$

Dando continuidade da eq. (4.4), encontra-se um máximo local, ou um mínimo global em $k=0$ dependendo se $\Omega$ é menor ou maior que $k_{L}^{2}$ respectivamente e, no caso de ser menor, outros dois mínimos locais, dados por

$$
\begin{aligned}
k_{ \pm}^{\prime} & = \pm k_{L} \sqrt{1-\left(\frac{\Omega}{k_{L}^{2}}\right)^{2}} \text { se } \Omega<k_{L}^{2} \\
k^{\prime} & =0 \text { se } \Omega>k_{L}^{2} .
\end{aligned}
$$

Isto mostra que o estado fundamental sofreria, na ausência de interações, uma transição diminuindo o momento até zero e assim permanece para todo $\Omega>k_{L}^{2}$.

Há ainda a possibilidade de ter uma sobreposição dos autoestados com $k_{+}^{\prime}$ e $k_{-}^{\prime}$, gerando uma modulação na densidade (interferência), que é conhecida como "striped phase" [13].

\section{Caso $\Omega=0$}

Nesta condição, o ramo de menor energia é uma aparente mistura dos ramos caso se desconsidere o módulo da raiz, e o espectro que contém a menor energia fica dado por

$$
\mathcal{E}(k)=\frac{k^{2}}{2}-\left|k_{L} k-\delta\right|,
$$

a qual possui dois mínimos

$$
\begin{gathered}
k_{+}^{\prime}=+k_{L} \quad \text { se } \quad k_{L} k-\delta>0, \\
k_{-}^{\prime}=-k_{L} \quad \text { se } \quad k_{L} k-\delta<0 .
\end{gathered}
$$


Contudo deve-se notar que a primeira delas só possui de fato solução caso $\delta<k_{L}^{2}$ e a segunda se $\delta>-k_{L}^{2}$, Isto porque o máximo local onde a função não é diferenciável se sobrepõe a um dos mínimos. Evitando essa complicação por hora, ou seja tratando $\delta \in\left(-k_{L}^{2}, k_{L}^{2}\right)$, podese notar que

$$
\begin{aligned}
& \mathcal{E}\left(k_{+}^{\prime}\right)=-\frac{k_{L}^{2}}{2}+\delta>\mathcal{E}\left(k_{-}^{\prime}\right)=-\frac{k_{L}^{2}}{2}-\delta \quad \text { se } \quad \delta>0, \\
& \mathcal{E}\left(k_{-}^{\prime}\right)=-\frac{k_{L}^{2}}{2}-\delta>\mathcal{E}\left(k_{+}^{\prime}\right)=-\frac{k_{L}^{2}}{2}+\delta \quad \text { se } \quad \delta<0 .
\end{aligned}
$$

Tais resultados comprovam o que já foi possível ver na figura $4.1 \mathrm{e}$, em palavras $\delta$ quebra a degenerescência entre os estados de momento positivo e negativo encontrados em (4.6). Já o efeito de $|\delta|>k_{L}^{2}$ é ilustrado na figura a seguir:

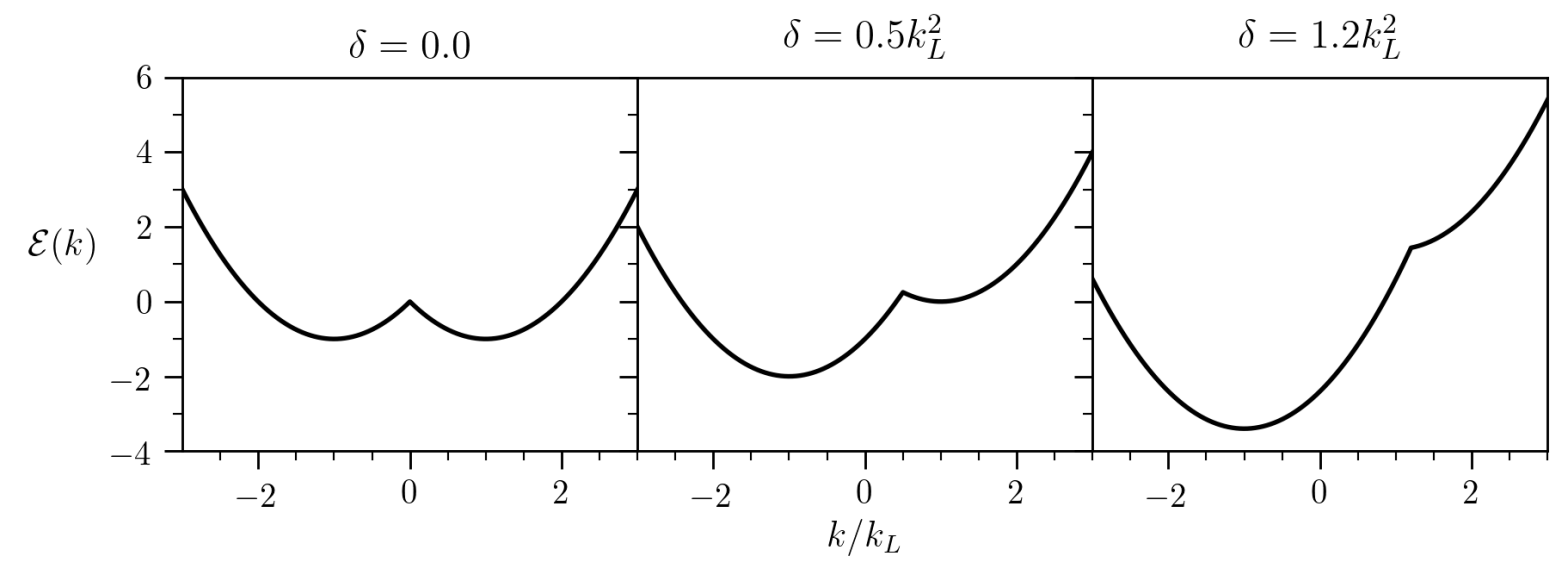

Figura 4.2: Energia para $k_{L}=\sqrt{2}$ e $\Omega=0$, para diferentes valores de $\delta$ explorando a transição de dois mínimos para apenas um mínimo.

\subsection{Estados Estacionários com Interação atrativa}

Com os métodos numéricos descritos no capítulo anterior, a obtenção de estados estacionários depende de uma tentativa inicial, a qual dita a convergência mais ou menos rápida. Contudo precisa-se de uma abordagem teórica para tanto.

Primeiramente ilustra-se a seguir as três fases distintas, baseado no trabalho [13], onde foi utilizado as formas analíticas presentes no artigo como condição inicial para as rotinas numéricas. Nos três casos, por anseio de compatibilidade, foram utilizados $\delta=0, k_{L}=8.0 \mathrm{e}$ $\beta=-0.8$ para interação entre estados de spin diferentes, igualmente ao artigo referido. 

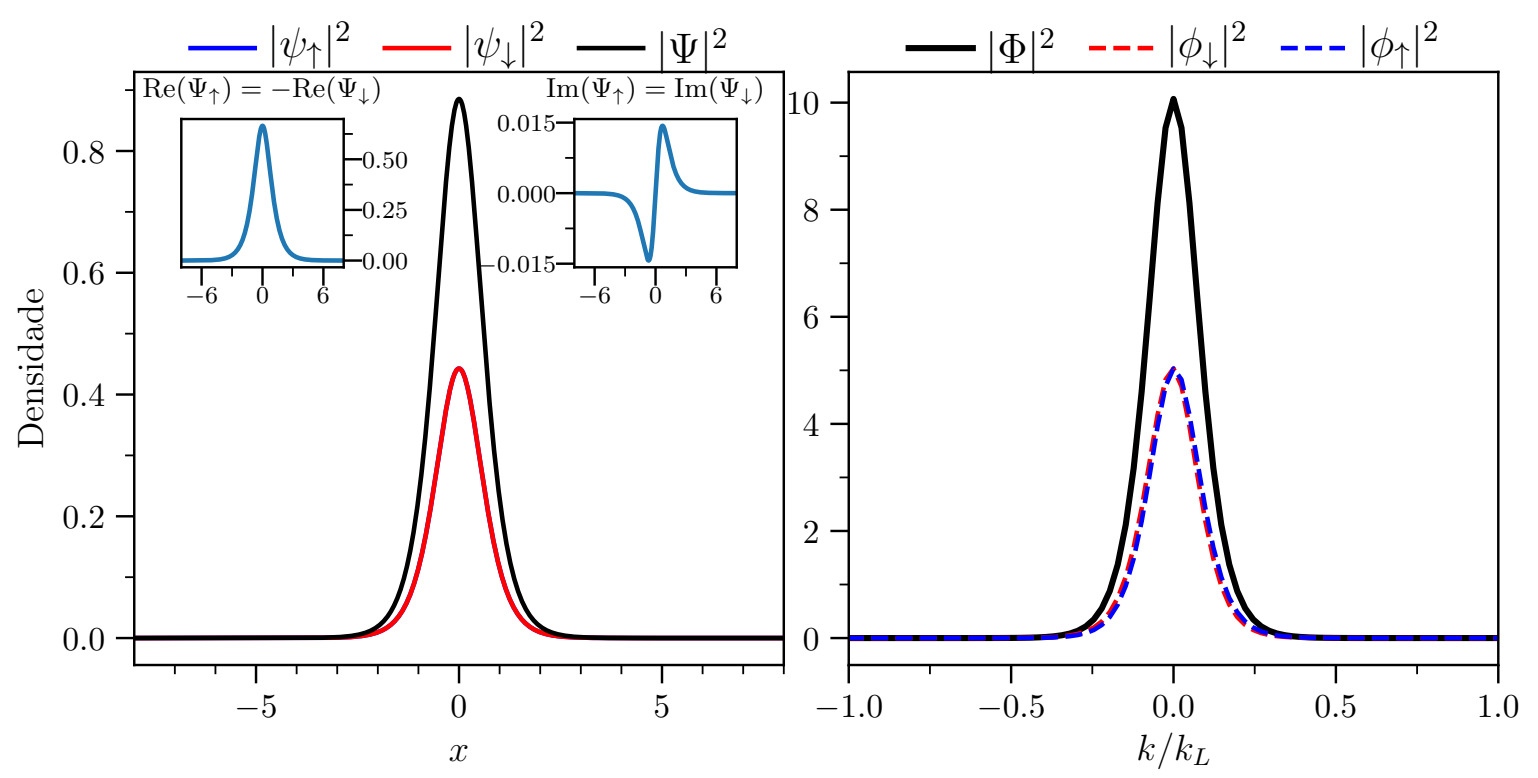

Figura 4.3: Para $\Omega=120$ e fixado $\mu=-120.4$, encontrou-se $E \simeq-120.13$ e $\mathcal{N} \simeq 1.36$, para energia média por átomo e normalização respectivamente. A figura mostra a densidade de átomos nas variáveis dinâmica posição(à esquerda) e momento(à direita) e, os gráficos contidos revelam simetria entre as componentes.
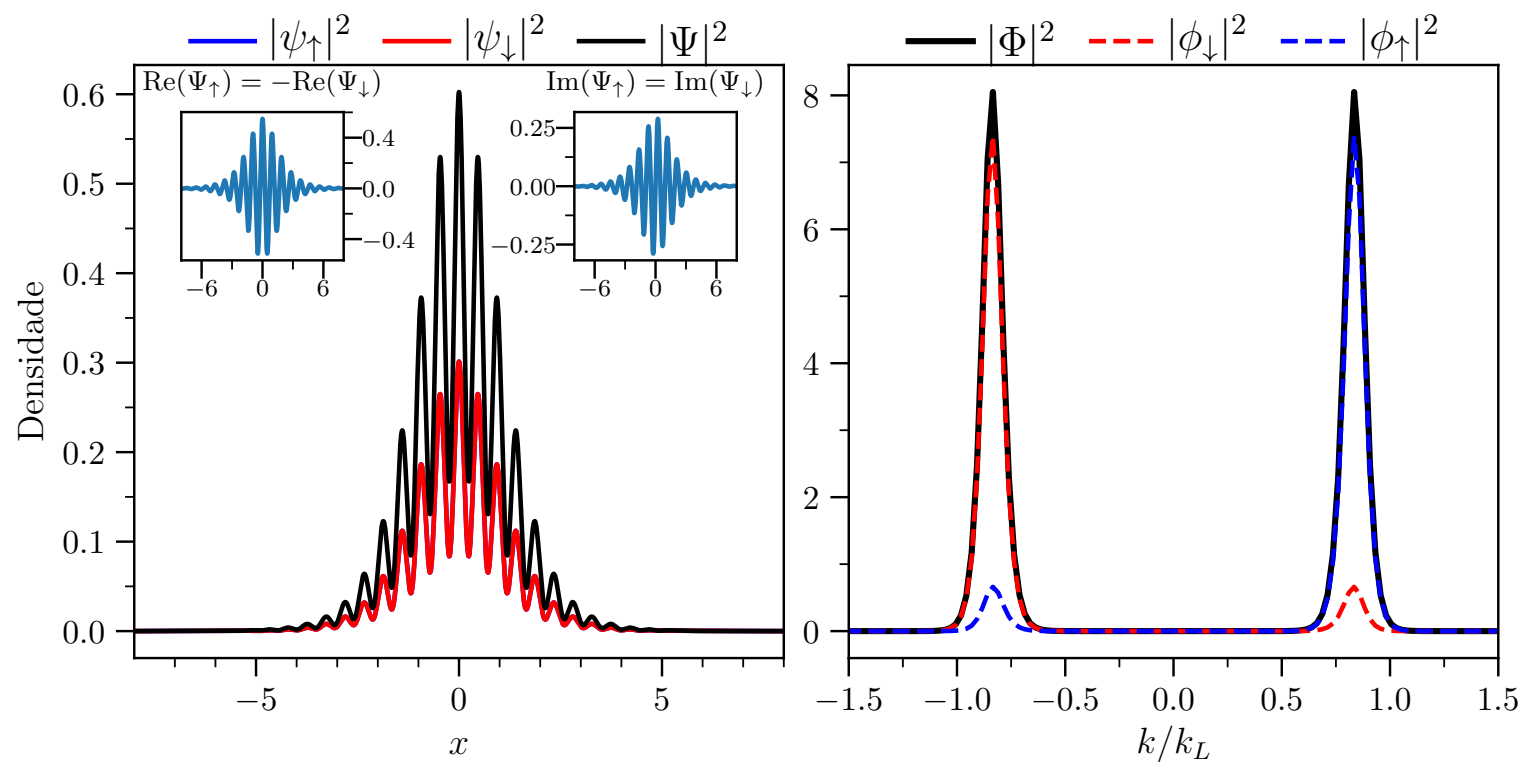

Figura 4.4: Para $\Omega=35$, fixado $\mu=-41.77$, obtém-se $E \simeq-41.64$ e $\mathcal{N} \simeq 1.02$ e verifica-se simetria entre componentes. A densidade no espaço de momentos mostra que cada população contribui mais para $+k^{\prime}(\uparrow)$ ou $-k^{\prime}(\downarrow)$, com $k^{\prime} \approx 0.813 k_{L}^{2}$, resultando contudo, numa distribuição igualmente balanceada, para densidade total, e ainda conduzindo a modulações na mesma.

Neste primeiro contato com estas fases, pode-se caracterizar suas diferenças em alguns observáveis. Na fig. 4.3 pelo balanceamento, i.e, $\mathcal{N}_{\uparrow}=\mathcal{N}_{\downarrow}$ e portanto é dita não magnetizada uma 

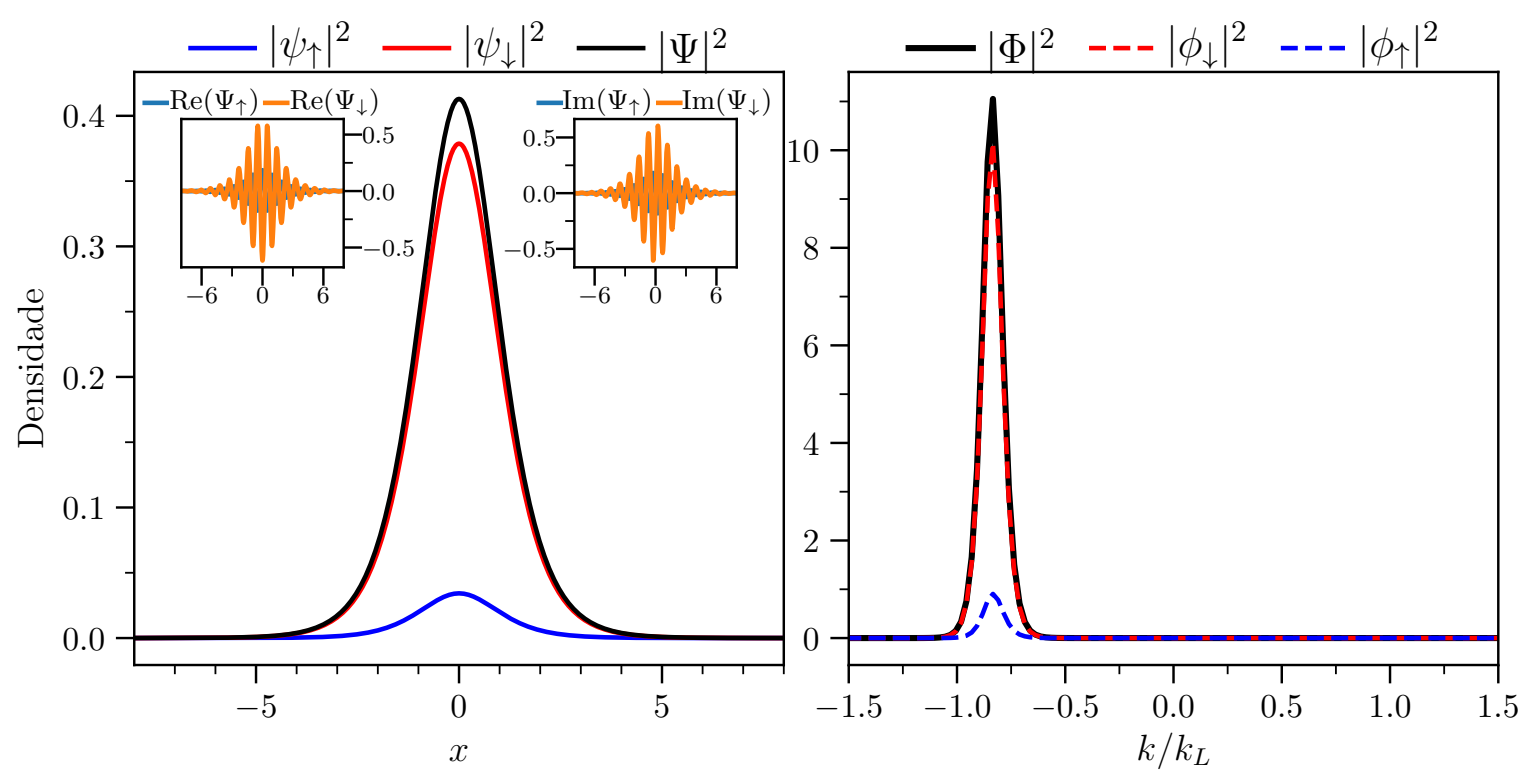

Figura 4.5: Para $\Omega=35$ e fixado $\mu=-41.77$. Encontrou-se $E \simeq-41.64$ e $\mathcal{N} \simeq 1.09$ e não há simetria entre as componentes, diferente dos dois outros anteriores. Ambas populações concentram-se distribuídas em $-k^{\prime}$, originando a fase chamada de "onda plana".

vez que $\left\langle\hat{\sigma}_{z}\right\rangle=\left(\mathcal{N}_{\uparrow}-\mathcal{N}_{\downarrow}\right) / \mathcal{N}=0$, exibindo além disso, valor esperado de momento total e individuais, $\langle k\rangle=\langle k\rangle_{\uparrow, \downarrow}=0$. Já na fig. 4.4, os observáveis relevantes que a caracterizam são a densidade no espaço de posição, e o contraste entre $\langle k\rangle=0$, embora $\langle k\rangle_{\uparrow, \downarrow} \neq 0$. Por fim, na fig. 4.5, contrasta em $\left\langle\hat{\sigma}_{z}\right\rangle \neq 0$ bem como $\langle k\rangle \neq 0$.

Vale ainda ressaltar que embora compartilhem o mesmo potencial químico $\mu$, a solução denominada "modulada"(striped) no gráfico 4.4 possui energia média por átomo ligeiramente maior que na fase de onda plana em 4.5 sendo mais precisamente as diferenças de: $E_{\mathrm{st}}-E_{\mathrm{pw}}=$ $2.61 \times 10^{-4},{ }^{1}$ contudo o número de átomos seja maior para fase de onda plana: $\mathcal{N}_{\mathrm{pw}}-\mathcal{N}_{\mathrm{st}}=$ $7.3 \times 10^{-2}$.

Um último comentário que cabe a essa seção é que a estabilidade, se comporta de maneira diferente variando o potencial químico na vizinhança dos valores usados, estudo ainda feito em [13], que concluiu haver uma região de estabilidade maior para a fase modulada frente a onda plana onde há $\mu_{c} \lesssim-42.9$ cuja esta última se torna instável.

\footnotetext{
1"pw" abreviação para plane wave e "st" abreviação para stripe
} 


\subsection{Estudo de Fases baseado em Cálculo Variacional}

A seção anterior, revela a sistemática em que os métodos desenvolvidos podem ser empregados para obter soluções estacionárias exatas, contudo deixa em aberto as transições entre uma e outra fase e o que ocorre com seus observáveis, sem fornecer detalhes da passagem de uma para outra.

Lançada a ideia de motivação, seguindo em linhas gerais [23] na seção 2.2, onde é proposto um Ansatz com parâmetros sujeitos a minimização no funcional de energia (2.102) após integração. Contudo neste artigo é feito tal procedimento com condições de contorno periódicas, e com certo pioneirismo propõe-se aqui uma modificação para um sistema atrativo da forma

$$
\Psi(x)=\frac{e^{-x^{2} / 4 \sigma^{2}}}{\left(2 \pi \sigma^{2}\right)^{1 / 4}}\left[C_{+}\left(\begin{array}{c}
\cos \theta \\
-\sin \theta
\end{array}\right) e^{i k x}+C_{-}\left(\begin{array}{c}
\sin \theta \\
-\cos \theta
\end{array}\right) e^{-i k x}\right] .
$$

Cabem alguns rápidos comentários sobre esta forma. A primeira é que o fator $k$ define o momento das componentes, e os coeficientes da superposição $C_{ \pm}$podem introduzir um desbalanço no número de átomos em cada estado de spin junto $\operatorname{com} \theta$, bem como fixar o momento em $\pm k$, ou uma sobreposição deles. Já o parâmetro $\sigma$ deve ser influenciado pela normalização, que já contém informação sobre a força de interação.

Por dizer na normalização, ocorre que ao integrar a forma acima proposta obtém-se

$$
\mathcal{N}=\int_{-\infty}^{\infty} \mathrm{d} x \Psi^{\dagger} \Psi=\left(C_{+} C_{-}^{*}+C_{-} C_{+}^{*}\right) \sin (2 \theta) e^{-2 k^{2} \sigma^{2}}+\left|C_{+}\right|^{2}+\left|C_{-}\right|^{2}
$$

No que decorre nos passos seguintes, fixa-se a norma e portanto a equação acima implica uma limitação a ser obedecida pelos parâmetros variacionais, $C_{+}, C_{-}, \theta, \sigma, k$. As integrações envolvidas, são detalhadas no apêndice B, para obtenção da energia como função dos parâmetros citados.

Com o funcional expresso em termos dos parâmetros, foi feito então a minimização com respeito a estes, mantendo a normalização em (4.14) fixa, variando os parâmetros da equação $k_{L}, \Omega$ e $\delta$, ou seja, para cada conjunto destes últimos três, obtemos cinco números para os parâmetros variacionais.

Após a minimização, que fornece valores numéricos para os parâmetros, pode-se então inserir a forma (4.13) com tais valores nas rotinas para obtenção da solução exata. Para o presente caso é utilizado o método de propagação em tempo imaginário descrito na seção 3.2.1, 
o qual nesse contexto é mais conveniente para manter a norma fixa.

A ênfase foi primeiramente dada para variar simultaneamente $k_{L}$ e $\Omega$, rendendo imagens com observáveis relevantes. O Parâmetro $\delta$ é então variado em passos fixos a cada imagem. Sobre as intensidades das interações foi respeitado primeiramente a simetria com relação a spin, i.e $\beta=-1.0$ e $\gamma=-1.0$, bem como a norma $\mathcal{N}=2.0$, com exceções mencionadas explicitamente.

Os observáveis escolhidos para mostrar foram o valor médio do módulo do momento, e não o momento total de $k$, isto porque caso fosse tomado simplesmente o momento médio, não haveria diferença entre as fases modulada(stripes) que se distribui simetricamente para $+k \mathrm{e}-k$ ou uma distribuição centrada em zero, como da figura 4.3. Vale dizer que tal medição coincide com o ponto máximo da distribuição como os casos das figuras 4.5 e 4.4 .

Além disso, como já foi mencionado, os parâmetros $C_{ \pm} \mathrm{e} \theta$ fazem o balanço entre a superposição de $-k \mathrm{e}+k \mathrm{e}$, é justamente este fator que caracteriza a aparição de modulações na densidade, as ditas stripes. Portanto pode-se definir uma correlação dos coeficientes presentes nesta superposição como

$$
\mathcal{C}=\frac{2\left|C_{+}\right|\left|C_{-}\right| \sin (2 \theta)}{\left|C_{+}\right|^{2}+\left|C_{-}\right|^{2}} ; \quad 0 \leq \mathcal{C} \leq 1 .
$$

Após estas descrição podemos construir os diagramas mostrados nesta seção.

Em todos eles foi mantido uma sistemática de análise, onde o mais marcante é a região branca onde deixou-se de mostrar o fator de correlação. A justificativa segue da própria natureza do sistema, pois para momentos menores que $\widetilde{k} \sim 1 / \sigma$, significa que a solução já decaiu exponencialmente a zero segundo um desvio padrão, sem portanto completar um ciclo na fase. Assim mesmo que as modulações no espaço de posições em tese ocorram devem apresentar até dois máximos locais, por exemplo. Quantitativamente foi decidido por $\widetilde{k} \doteq \pi /(2 \bar{\sigma})$, com $\bar{\sigma}$ a média sobre os diferentes valores de $\sigma$, do variacional, com respeito a $\Omega$.

A leitura das imagens 4.6, 4.7e 4.8 feitas de forma sobreposta, entre o módulo do momento esperado e a correlação dos coeficientes variacionais, mostram que se a função de Correlação for nula (azul escuro) não podem ocorrer modulações e significa que temos um estado em que as populações $(\uparrow, \downarrow)$ descrevem uma distribuição no espaço de momentos centrada em $+|k|$ ou $-|k|$ mas não em ambos simultaneamente.

O que conclui-se da leitura, e o que condiz com a diagonalização da Hamiltoniana sem interações é que o fator de "detuning" $(\delta$ ) favorece a fase de onda plana frente a modulada, pelo predomínio da região em azul escuro nas cores indicando o valor da correlação segundo a eq. 


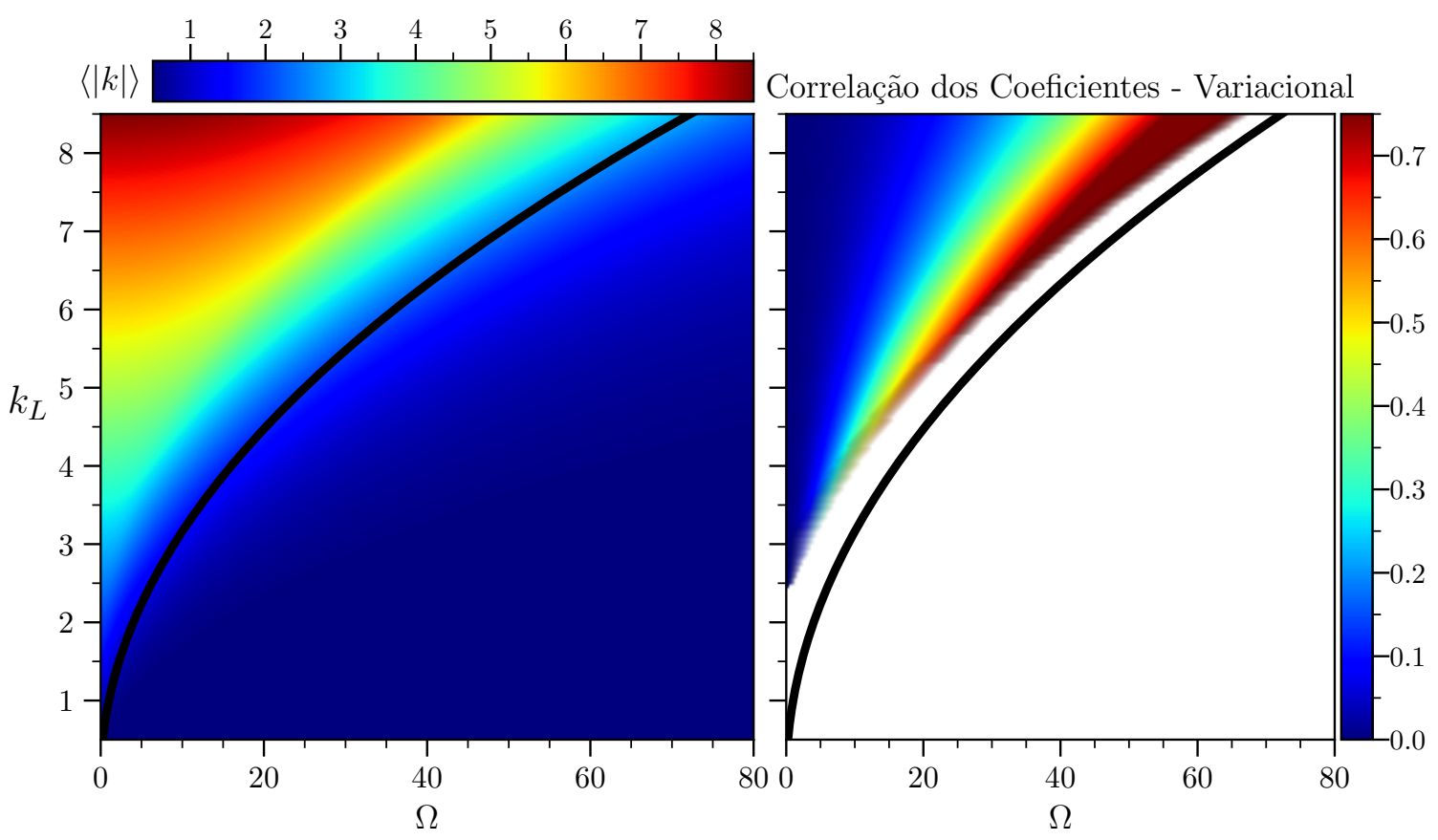

Figura 4.6: Para $\delta=0$, em ambas variando os parâmetros da equação $k_{L}$ e $\Omega$, obtém-se os observáveis descritos no texto representados em um mapeamento de cores. A linha em preto mostra a transição da região onde ocorre dois mínimos simétricos com momento $k^{\prime}=$ $\pm k_{L} \sqrt{1-\left(\Omega / k_{L}^{2}\right)^{2}}$ na eq. (4.6) e um único mínimo em $k^{\prime}=0$ prevista pela diagonalização sem interação. A direita a zona branca foi decidida pelo critério $\langle|k|\rangle\langle\pi /(2 \bar{\sigma})$, com $\bar{\sigma}$ a média do desvio padrão do cálculo variacional sobre os valores de $\Omega$.

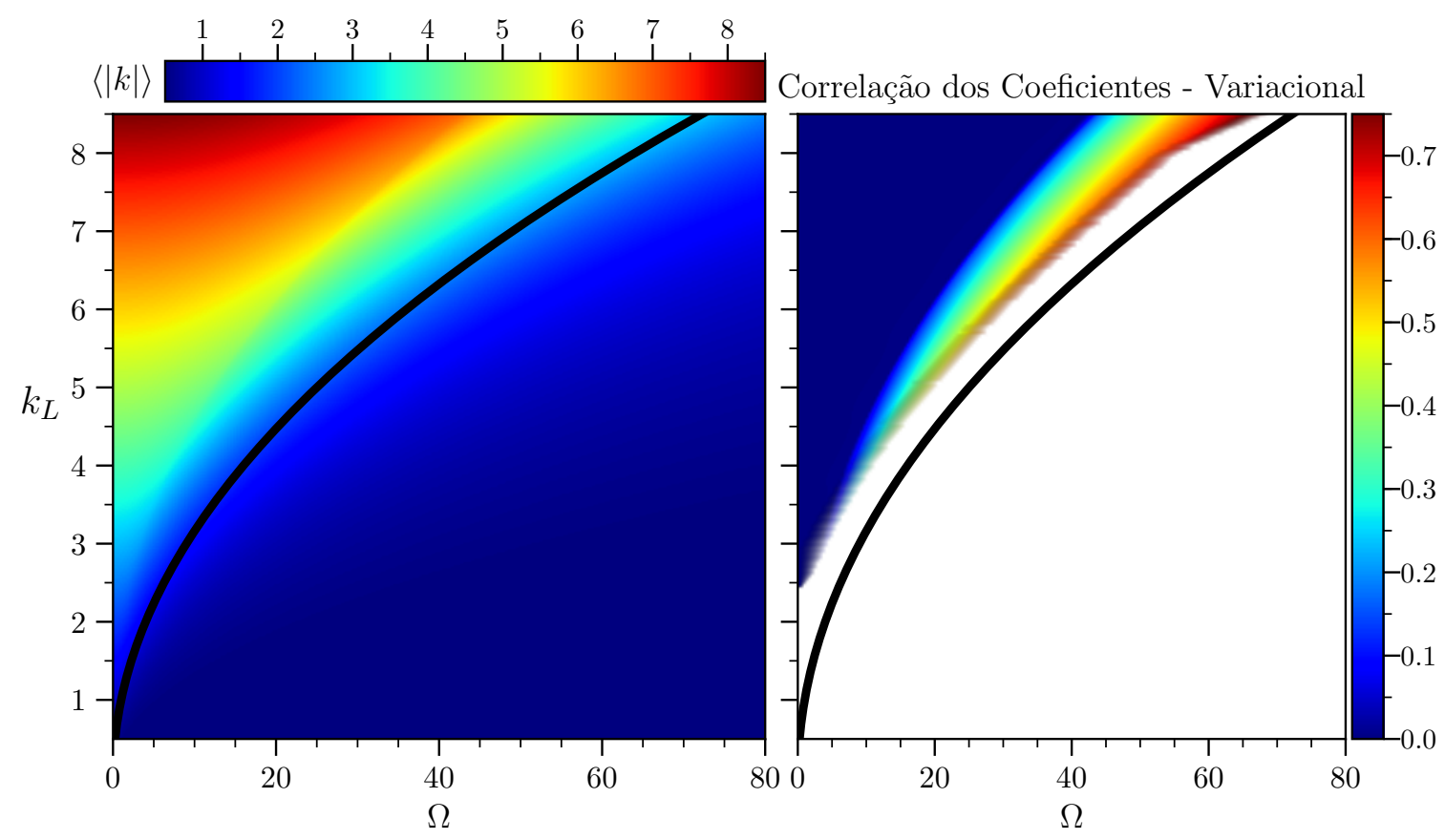

Figura 4.7: Para $\delta=0.2$ com a mesma sistemática da figura anterior. A linha em preto continua sendo com respeito ao resultado analítico para $\delta=0$ por fins comparativos. A zona pintada de branco continua a respeitar $\langle|k|\rangle<\pi /(2 \bar{\sigma})$ 


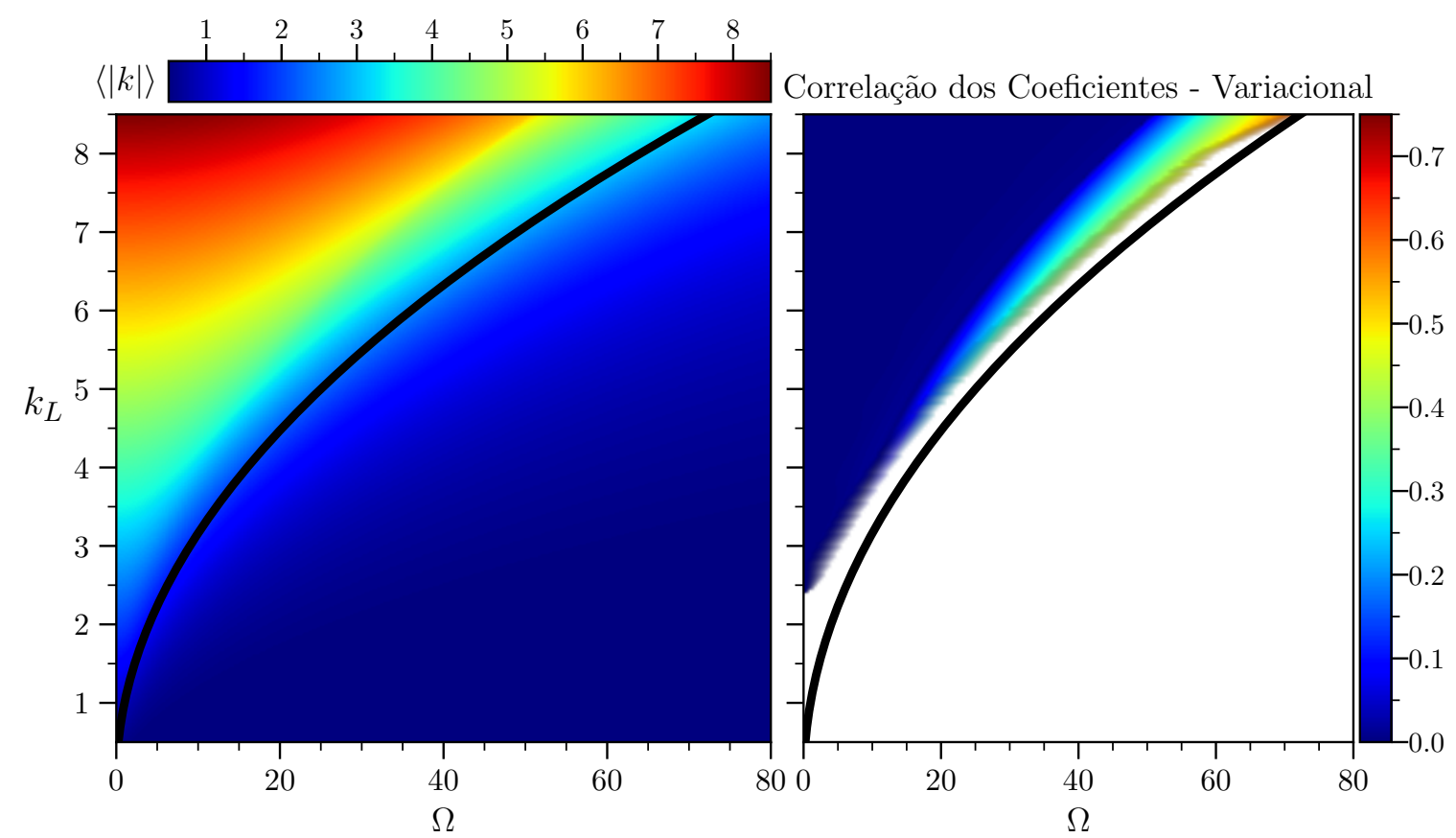

Figura 4.8: Diagrama para $\delta=1.0$. Outros parâmetros são como na fig. 4.6

(4.15), por criar uma assimetria entre os dois mínimos, já previsto na diagonalização exata sem interação.

\subsubsection{Diagramas com $k_{L}$ fixado}

Os diagramas anteriores, embora um tanto gerais, não revelam de forma fácil os valores e nem exploram outros observáveis relevantes. Para complementar a análise fixou-se então $k_{L}$, como se tomássemos uma linha sobre as imagens anteriores, onde será mostrado a seguir, um conjunto mais completo dos observáveis.

No que segue foram utilizadas as mesmas simulações, ou seja, preservando $\mathcal{N}=2.0$ e os coeficientes de interação, da seção precedente $\beta=-1.0$ e $\gamma=-1.0$. Fixa-se então adicionalmente $k_{L}=7.5$ e, deixa-se $\Omega$ como parâmetro livre, além de $\delta$ variando em passos entre as imagens.

Adicionou-se uma análise mais detalhada dos momentos, separando o de cada população $\langle k\rangle_{\uparrow, \downarrow}$ e o total $\langle k\rangle$, sem deixar de lado o módulo que já vinha da análise anterior. Simultaneamente mostra-se também a magnetização, que traz junto informações sobre o números de átomos em cada estado de spin, se estão ou não balanceados.

Um conjunto de gráficos é mostrado a direita de cada caso, forçando a saída da solução exata renormalizando as populações, mas sem mudar a forma funcional. Por exemplo seja $\mathcal{N}_{\uparrow, \downarrow}$ 

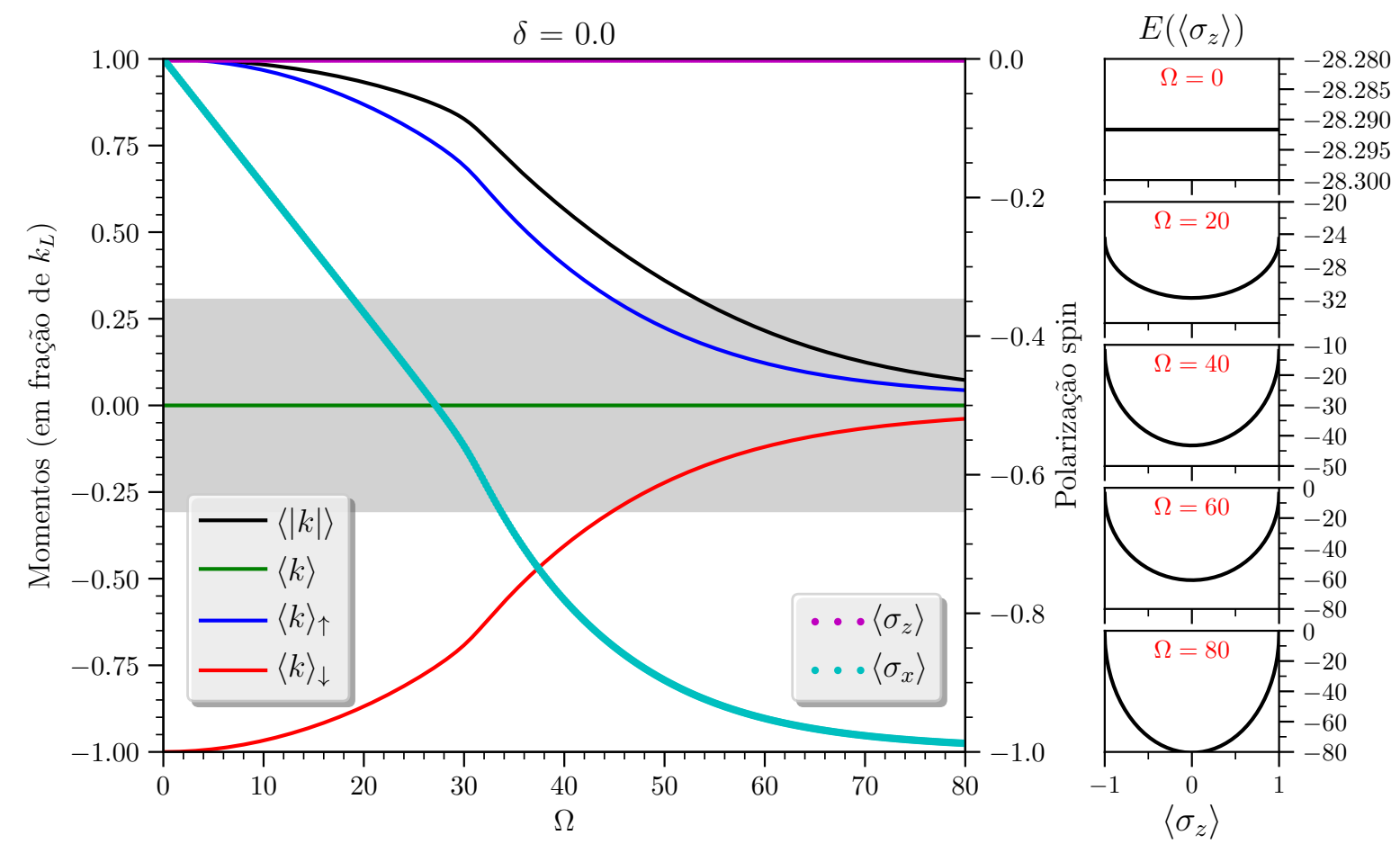

Figura 4.9: Com $k_{L}=7.5$ mostra-se diferentes medições dos momentos, cujo os valores são lidos no eixo à esquerda e, simultaneamente a magnetização $\left\langle\hat{\sigma}_{x, z}\right\rangle$ com os valores no eixo a direita. A coluna ao lado é obtida variando a normalização individual de cada população, mas mantendo a forma funcional, onde é possível ver o mínimo explícito da energia, mencionado previamente, com exceção em $\Omega=0$, que será discutido adiante. A faixa cinza corresponde a momentos, em módulo, inferiores a $\widetilde{k}=\pi /(2 \bar{\sigma})$, discutido no texto.

as normas respectivas da solução exata, então faz-se $\psi_{\uparrow, \downarrow}^{\prime}=\sqrt{\mathcal{N}_{\uparrow, \downarrow}^{\prime} / \mathcal{N}_{\uparrow, \downarrow}} \psi_{\uparrow, \downarrow}$, ainda respeitando $\mathcal{N}_{\uparrow}^{\prime}+\mathcal{N}_{\downarrow}^{\prime}=\mathcal{N}$ a norma total. Essa variação é mostrada, contudo em função de $\left\langle\hat{\sigma}_{z}\right\rangle^{\prime}=\left(\mathcal{N}_{\uparrow}^{\prime}-\right.$ $\left.\mathcal{N}_{\downarrow}^{\prime}\right) / \mathcal{N}$ e, será possível ver o mínimo sobre o valor exato $\left\langle\hat{\sigma}_{z}\right\rangle$, tirado do gráfico a esquerda para o respectivo valor de $\Omega$, que é representado nos subgráficos a direita com uma linha vertical roxa, ligando ao mínimo.

Em sequência das figuras dos observáveis do estado fundamental, é detalhado um conjunto de gráficos mostrando a densidade total no espaço de posição e de momentos, este último medido em unidades de $k_{L}$, para alguns valores de $\Omega$.

Uma característica que as imagens a seguir compartilham é uma faixa em cinza, que corresponde a uma região onde a densidade é essencialmente zero devido a forma gaussiana proposta. Do Ansatz (4.13) feito para minimização do variacional, o parâmetro $\sigma$ determina tal região, contudo através de $\widetilde{k} \doteq \pi /(2 \bar{\sigma})$, conduzindo a no máximo uma oscilação antes do decaimento exponencial da gaussiana dominar. Como os valores de $\sigma$ variam para cada valor de $\Omega$, toma-se então a média sobre todos $\Omega$, denotado por $\bar{\sigma}$, assim como usado anteriormente. 

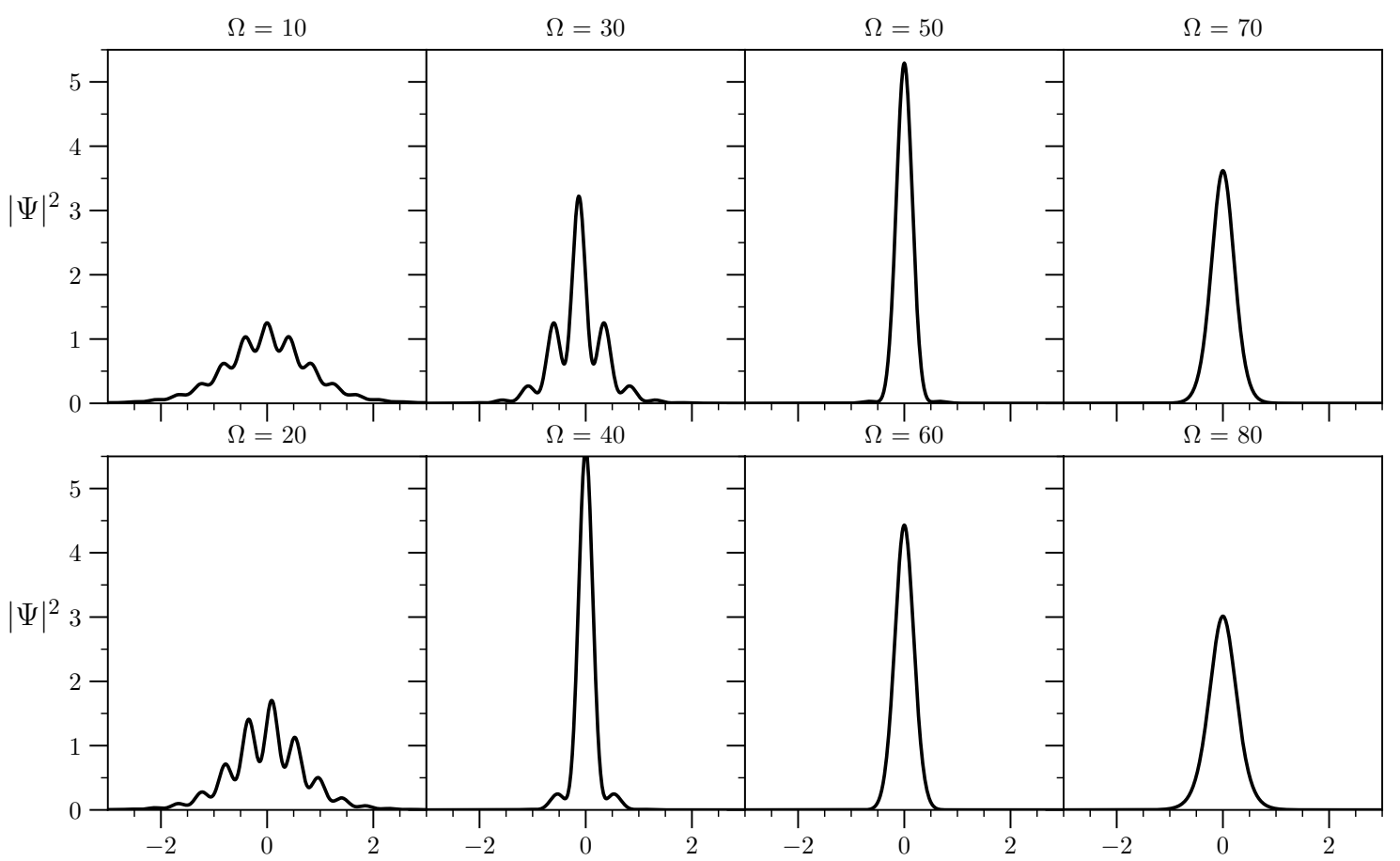

(a) Densidades no espaço de posições
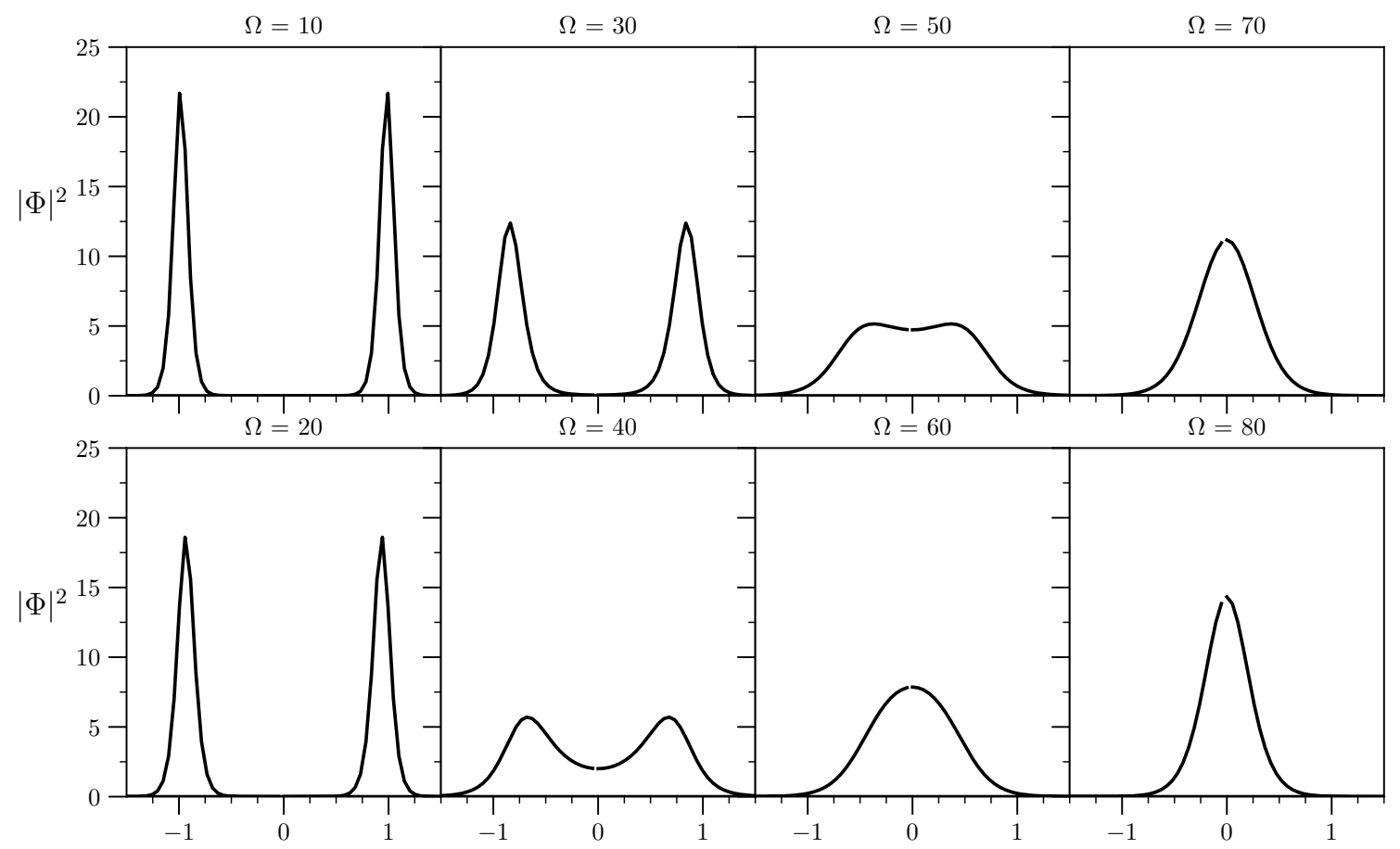

(b) Densidades no espaço de momentos

Figura 4.10: Ainda referente $\delta=0$ e $k_{L}=7.5$ a forma funcional da densidade do estado fundamental para alguns valores de $\Omega$, no espaço de posição (a) e de momento (b).

Analisando todo o conjunto de figuras de 4.9 a 4.14, podemos contrastar primeiro o efeito de deslocar $\delta$ de zero, que produz uma "preferência" em termos de energia para uma das populações, que para o presente caso vem a ser para $(\downarrow)$ uma vez variado $\delta>0$. Este fato 


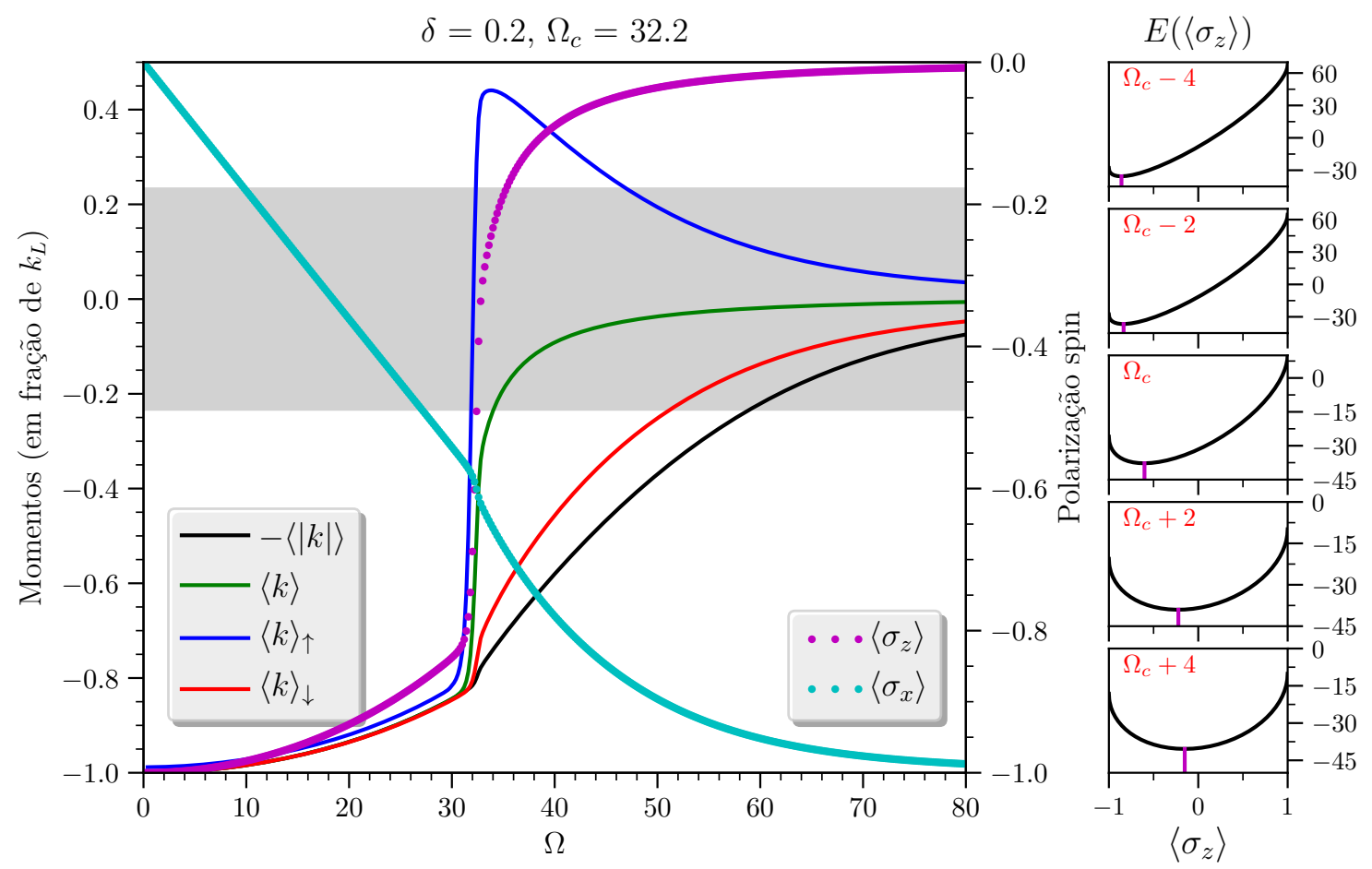

Figura 4.11: Como anteriormente $k_{L}=7.5$, no entanto aparece agora uma variação brusca para um certo valor de $\Omega$ denominado crítico, que representa um máximo na derivada com respeito a $\left\langle\hat{\sigma}_{z}\right\rangle$ e, uma parente descontinuidade com respeito a derivada de $\left\langle\hat{\sigma}_{x}\right\rangle$, o qual o valor é encontrado no topo do gráfico. Desta vez, note que foi mostrado $-\langle|k|\rangle$, em fim de ilustrar a perfeita sobreposição com $\langle k\rangle_{\downarrow}$ para $\Omega<\Omega_{c}$. Como usual a faixa cinza corresponde a momentos inferiores a $\widetilde{k}=\pi /(2 \bar{\sigma})$

se destaca para uma região $\Omega \lesssim \Omega_{c}$, detalhado nas imagens.

Na região $\Omega \gtrsim \Omega_{c}$ a energia passa a ser dominada por $\left\langle\hat{\sigma}_{x}\right\rangle$ e $\delta$ torna-se menos relevante na descrição, conduzindo o estado fundamental a uma distribuição centrada em zero no espaço de momentos, com populações balanceadas $\left(\left\langle\hat{\sigma}_{z}\right\rangle \approx 0\right)$ e polarizada na direção do acoplamento spin-órbita, com sentido oposto $\left(\left\langle\hat{\sigma}_{x}\right\rangle \approx-1\right)$.

Por outro lado, mostrar de fato a energia ao redor do valor onde ocorre o mínimo com relação a um parâmetro(como discutido as normas de cada espécie, caracterizado por $\left\langle\hat{\sigma}_{z}\right\rangle$ ), é interessante pois eventualmente temos mais mínimos locais, que se tornam globais com a variação de certos parâmetros. Contudo não é isso que ocorre simplesmente rebalanceando os estados em suas componentes $(\uparrow),(\downarrow)$, para todos os casos estudados, confirmado pelos pequenos gráficos a direita.

Uma característica compartilhada, é o estreitamento da densidade no espaço de posição para valores típicos de $40 \lesssim \Omega \lesssim 50$. O efeito de $\delta$ neste caso é sutil, mostrando que deve depender mais fortemente de uma transição baseada em $k_{L}$ e $\Omega$. 

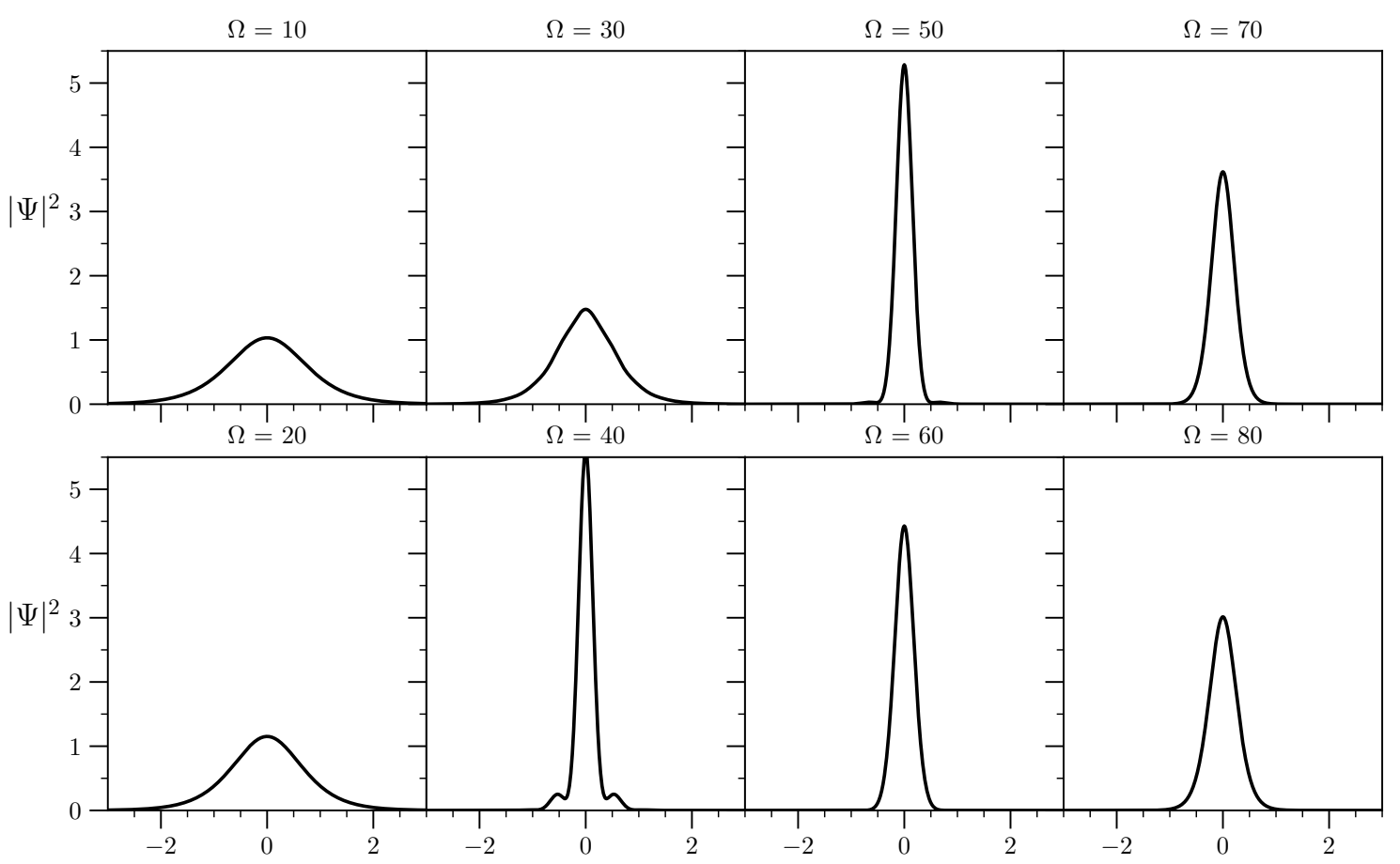

(a) Densidades no espaço de posições
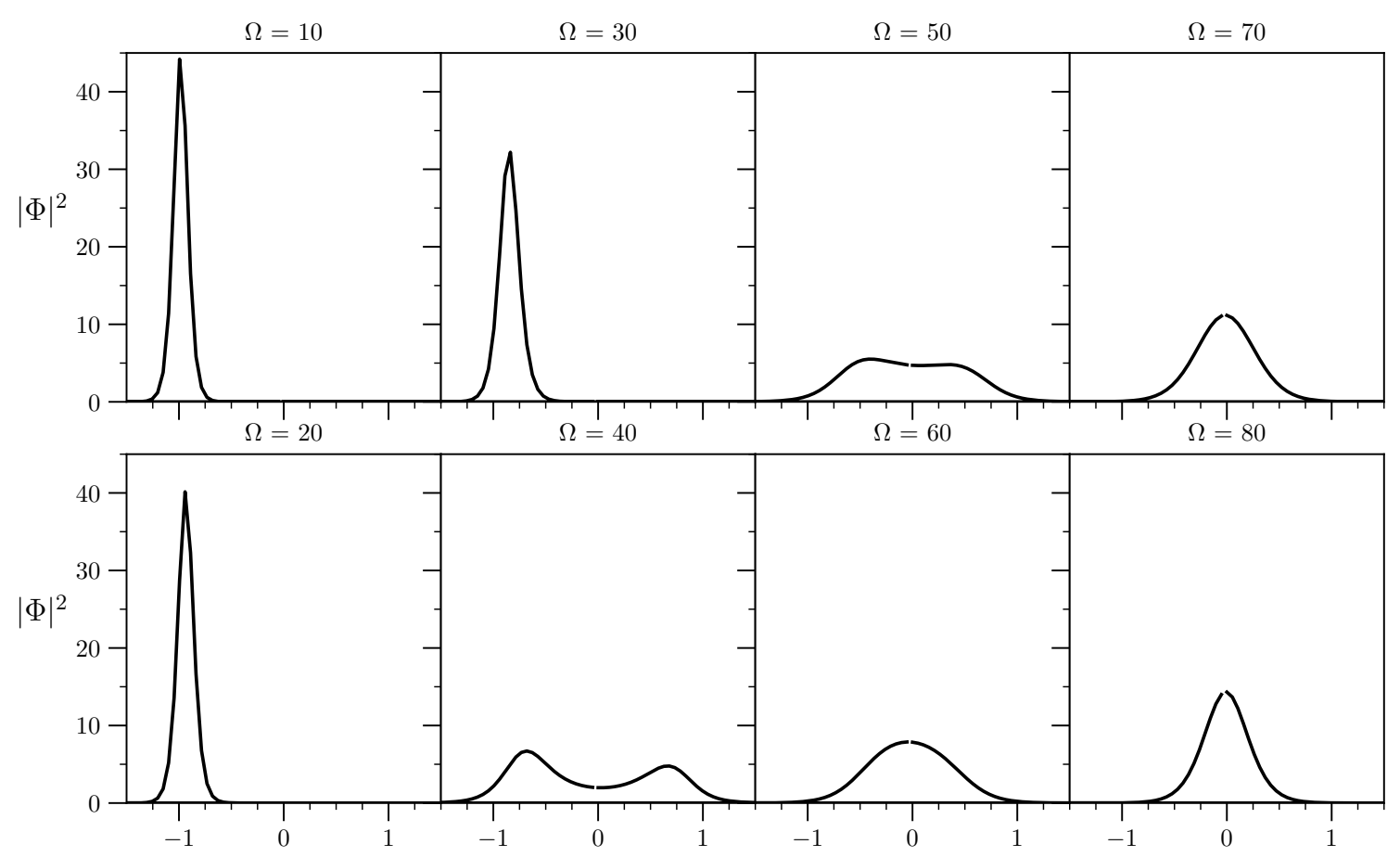

(b) Densidades no espaço de momentos

Figura 4.12: Referente $\delta=0.2$ e $k_{L}=7.5$ a forma funcional da densidade do estado fundamental para alguns valores de $\Omega$. Nota-se a atenuação da fase modulada se comparado a $\delta=0$, mas residual ainda na transição em torno de $\Omega=40$. Além disso a nítida fase de onda plana para $\Omega \lesssim 30$, o que condiz com o mínimo na diagonalização sem interação, vide figuras 4.1 e 4.2 que possui um mínimo para $k<0$. 


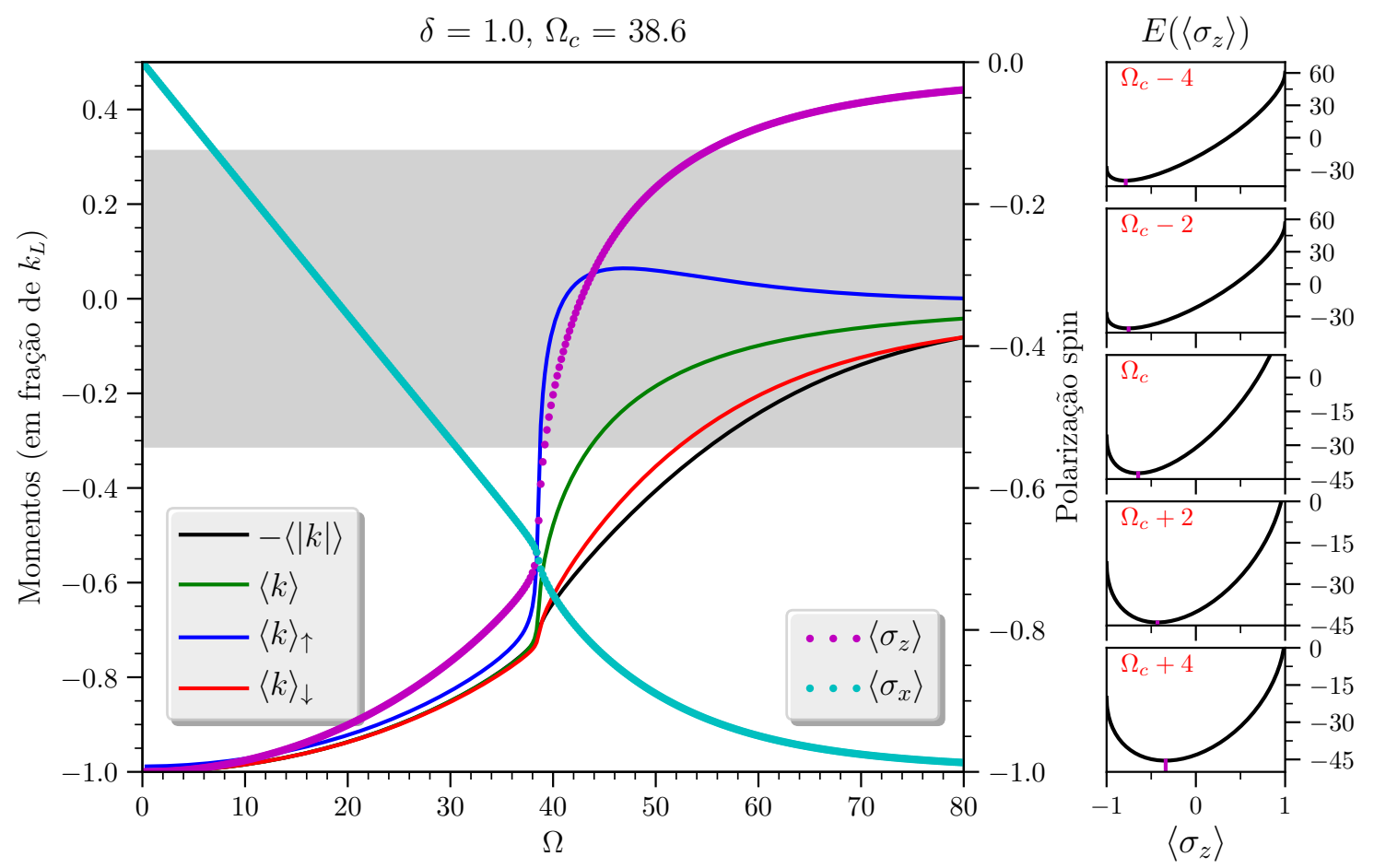

Figura 4.13: Demonstra características, em geral análogas a fig. 4.11. No entanto $\Omega_{c}$ onde se apresenta um máximo na derivada de $\left\langle\hat{\sigma}_{z}\right\rangle$ é ligeiramente maior e os observáveis variam de maneira menos brusca.

Por fim para os casos já mostrados e adicionalmente $\delta=0.5$, é exibida a energia do estado fundamental como função do parâmetro $\Omega$ na fig. 4.15 onde pode-se notar uma leve diferença baseada em $\delta$ apenas para $\Omega \approx 0$.

\subsubsection{Efeito de $\delta$ sobre o Estado Fundamental}

Como até então, $\delta$ variou em passos fixos, não foi explorado até aqui uma situação onde este parâmetro tem um efeito mais predominante sobre a caracterização do estado fundamental, muito embora, sua pouca influência foi suficiente para suprimir a fase modulada(striped).

Motivado pela primeira realização experimental de um Condensado de Bose-Einstein com acoplamento Spin-Órbita em uma direção feito em [17], fora feito de forma muito semelhante a figura três desta última citação, onde fixou-se os parâmetros $k_{L}$ e $\Omega$ em valores apreciavelmente mais baixos dos tratados até agora e, variou-se $\delta$.

É interessante contrastar [17] com o resultado na fig. 4.16 que revelam exatamente a mesma forma. 

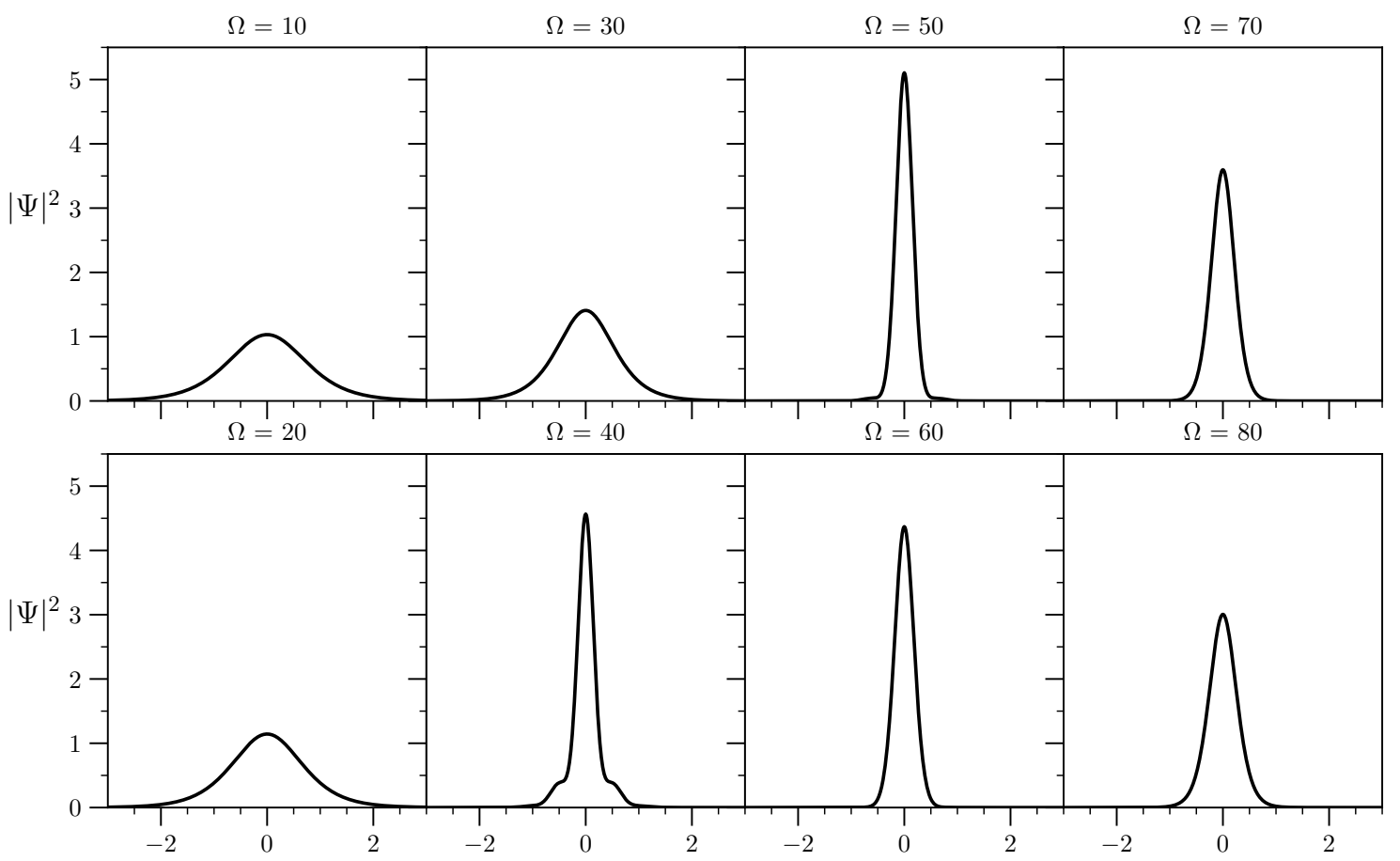

(a) Densidades no espaço de posições
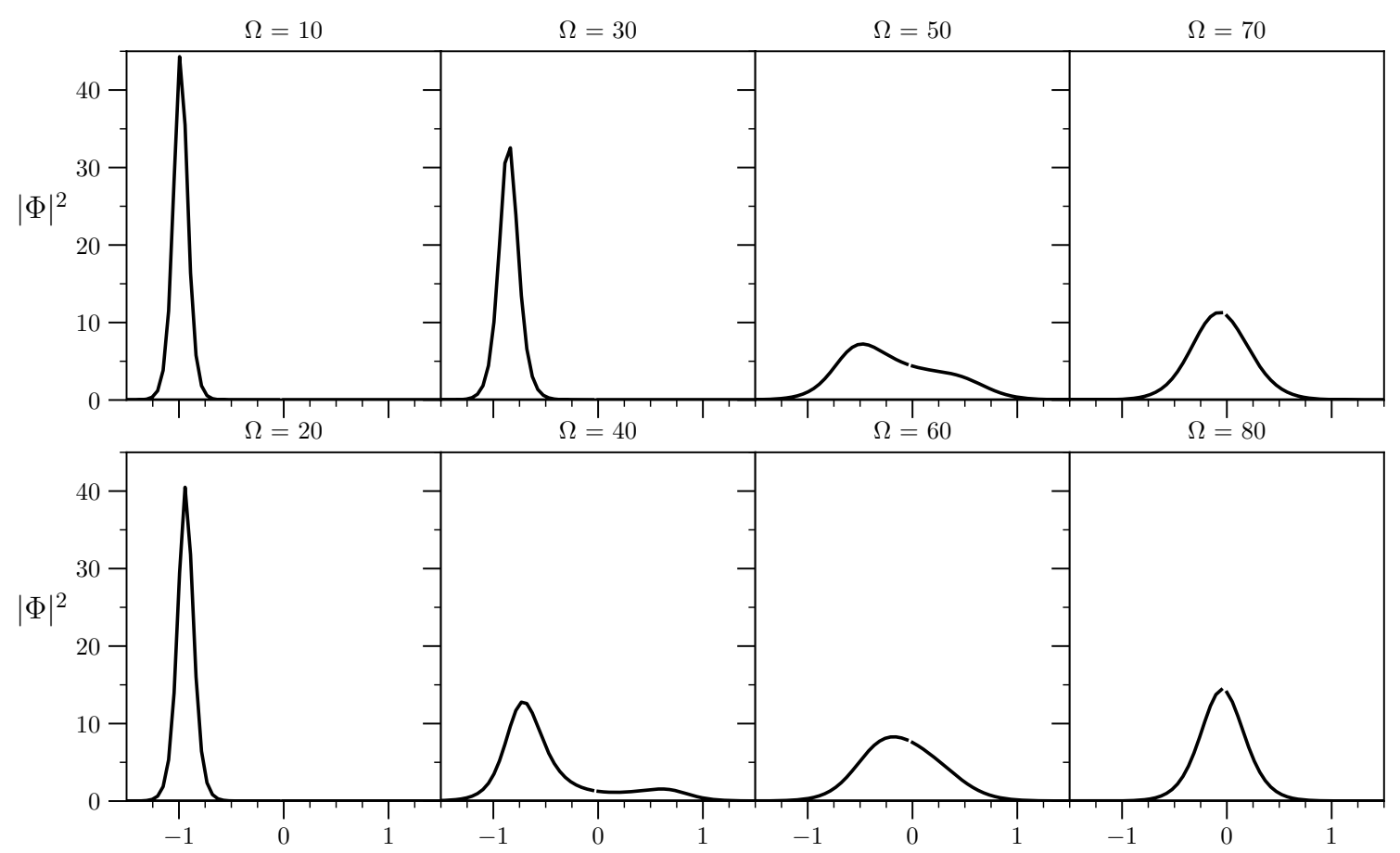

(b) Densidades no espaço de momentos

Figura 4.14: Referente $\delta=1.0$ e $k_{L}=7.5$ a forma funcional da densidade do estado fundamental para alguns valores de $\Omega$. Demonstra as mesmas características de $\delta=0.2$ na fig. 4.12, mas com uma atenuação maior das "stripes". 


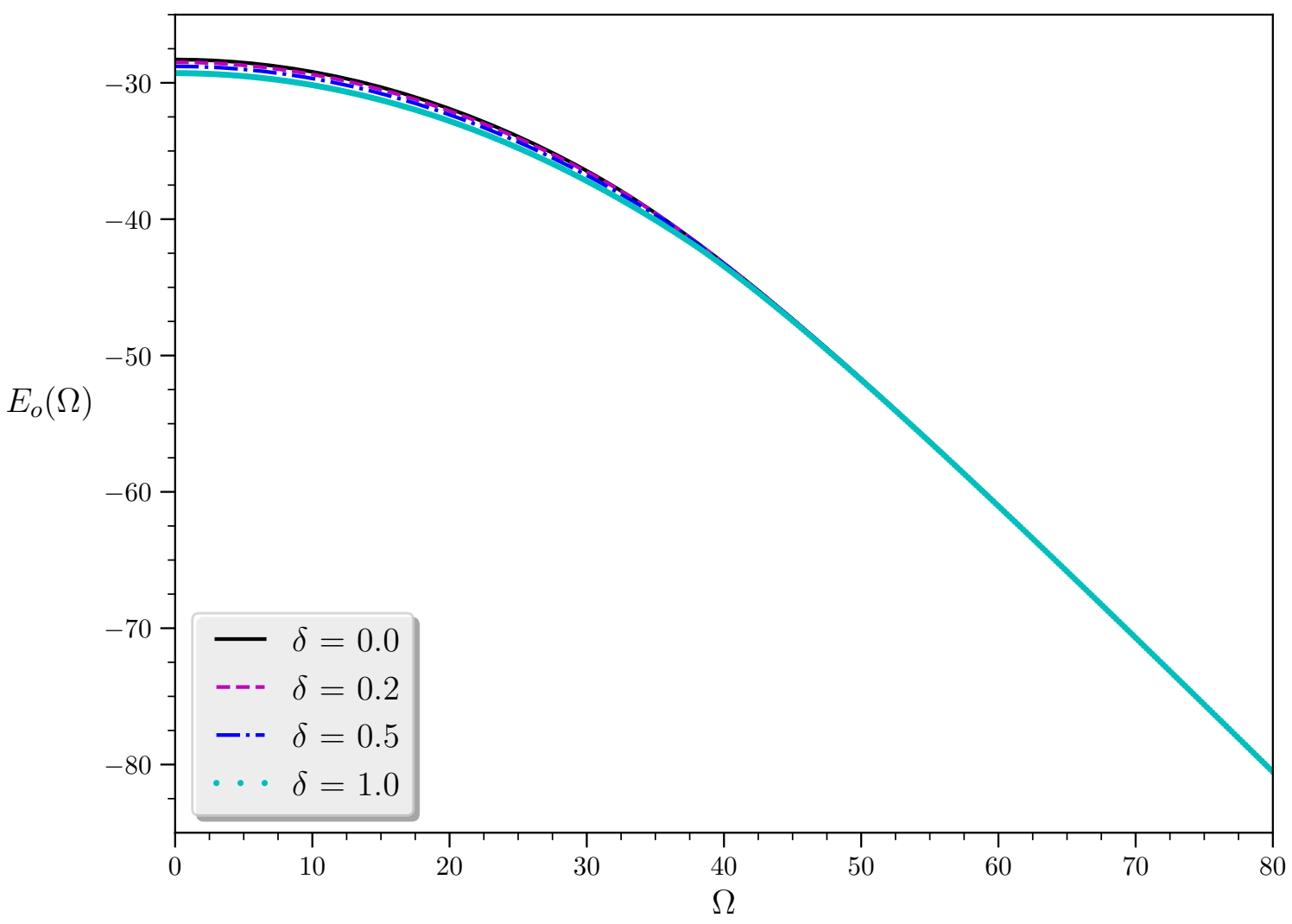

Figura 4.15: Energia do estado fundamental $E_{o}$, com $k_{L}=7.5$ para diferentes valores aqui trabalhados de $\delta$, como função de $\Omega$. Note que para energia não há nenhuma aparente descontinuidade, nem mesmo em sua derivada, quando comparada aos outros observáveis das figuras anteriores.
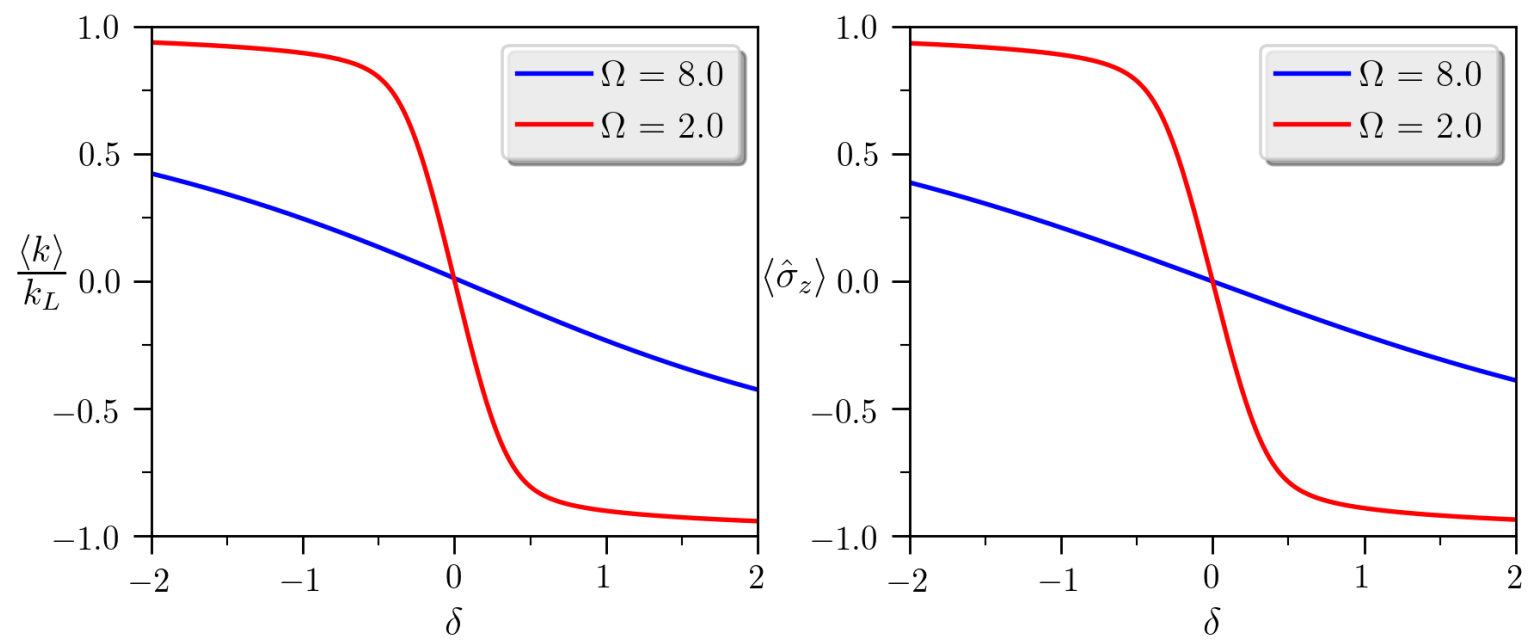

Figura 4.16: Para $k_{L}=2.0$ mostra-se a magnetização, que leva a concluir também sobre o número de átomos em cada estado de spin a direita. A esquerda o valor médio do momento em frações de $k_{L}$. A forma de ambas que são quase idênticas, mostram que $\delta$ dita a polarização juntamente com a entrada na fase de onda plana sendo de forma mais crítica para valores pequenos de $\Omega$ comparado a $k_{L}^{2}$ 


\subsection{Dinâmica de Sólitons}

O estudo de soluções do tipo sóliton, são recorrentes em equações não lineares com interação atrativa, que significa um conjunto de átomos auto-confinados, mesmo na ausência de potencial para armadilhar.

Muito embora para o caso geral $\operatorname{com} \Omega, k_{L} \neq 0$ o sistema é dito não integrável [14], cabe uma abordagem variacional, minimizando a ação (2.65). Tal abordagem se inicia com a forma

$$
\left(\begin{array}{c}
\psi_{\uparrow} \\
\psi_{\downarrow}
\end{array}\right)=\sqrt{\frac{\mathcal{N} \eta}{2}}\left(\begin{array}{l}
\sin (\theta) \operatorname{sech}\left(\eta x+\xi_{\uparrow}\right) e^{i\left(k_{\uparrow} x+\phi_{\uparrow}\right)} \\
\cos (\theta) \operatorname{sech}\left(\eta x+\xi_{\downarrow}\right) e^{i\left(k_{\downarrow} x+\phi_{\downarrow}\right)}
\end{array}\right),
$$

cujo os parâmetros $\eta, \theta, \xi_{\lambda}, k_{\lambda}, \phi_{\lambda}$ carregam dependência temporal, no entanto mantendo a normalização fixa em $\mathcal{N}$, conservando assim o número total de átomos.

Cabe aqui um comentário, que na abordagem acima, abdica-se de uma sobreposição de momentos, como feito em (4.13), a qual não pode conduzir portanto a nenhuma modulação na densidade(stripes).

Os resultados são analíticos para as equações de movimento, i.e, para que seja possível fazer as integrações da lagrangiana, se $\xi_{\uparrow}=\xi_{\downarrow}=\xi$, que é o caso tratado no apêndice A. Neste mesmo apêndice é mencionado a condição para que esta solução não sofra dispersão, que aqui será estudada, dada por

$$
k_{-}=k_{L}, \forall t, \quad \mathcal{N} \gg 2 \pi k_{L}
$$

ainda considerando a simetria de interação $|\beta|=1$. Para este regime, denotando ainda $\widetilde{\Omega}=$ $\Omega z / \sinh (z)$, onde $z=\pi k_{-} / \eta$, as equações do apêndice tornam-se

$$
\begin{aligned}
\langle\dot{x}\rangle & =k_{+}, \\
\dot{\theta} & =-\widetilde{\Omega} \sin \left(2 \phi_{-}+2 k_{L}\langle x\rangle\right), \\
\dot{k}_{+} & =\cos (2 \theta)+2 k_{L} \widetilde{\Omega} \sin (2 \theta) \sin \left(2 \phi_{-}+2 k_{L}\langle x\rangle\right), \\
\dot{\phi}_{-} & =-\delta-\widetilde{\Omega} \cot (2 \theta) \cos \left(2 \phi_{-}+2 k_{L}\langle x\rangle\right), \\
\eta & =\frac{\mathcal{N}}{2} .
\end{aligned}
$$

É válido comentar, que estas equações traçam semelhanças com outros trabalho como em [43], podendo reproduzir as equações lá presentes a partir deste variacional, com certa escolha dos parâmetros, detalhada no apêndice A. 
Um estudo preliminar das equações acima, mostra que para estados inicialmente balanceados, ou seja, $\theta_{o}=\pi / 4$, sem momento total inicial $k_{o+}$, centrado inicialmente em zero, $\xi=\langle x\rangle_{o}=0$ além de $2 \phi_{o_{-}}=\pi$, que a única equação que teria derivada não nula é $\dot{\phi}_{o_{-}}=-\delta$, concluindo então que no caso $\delta=0$, deve-se então se manter na realidade estacionário.
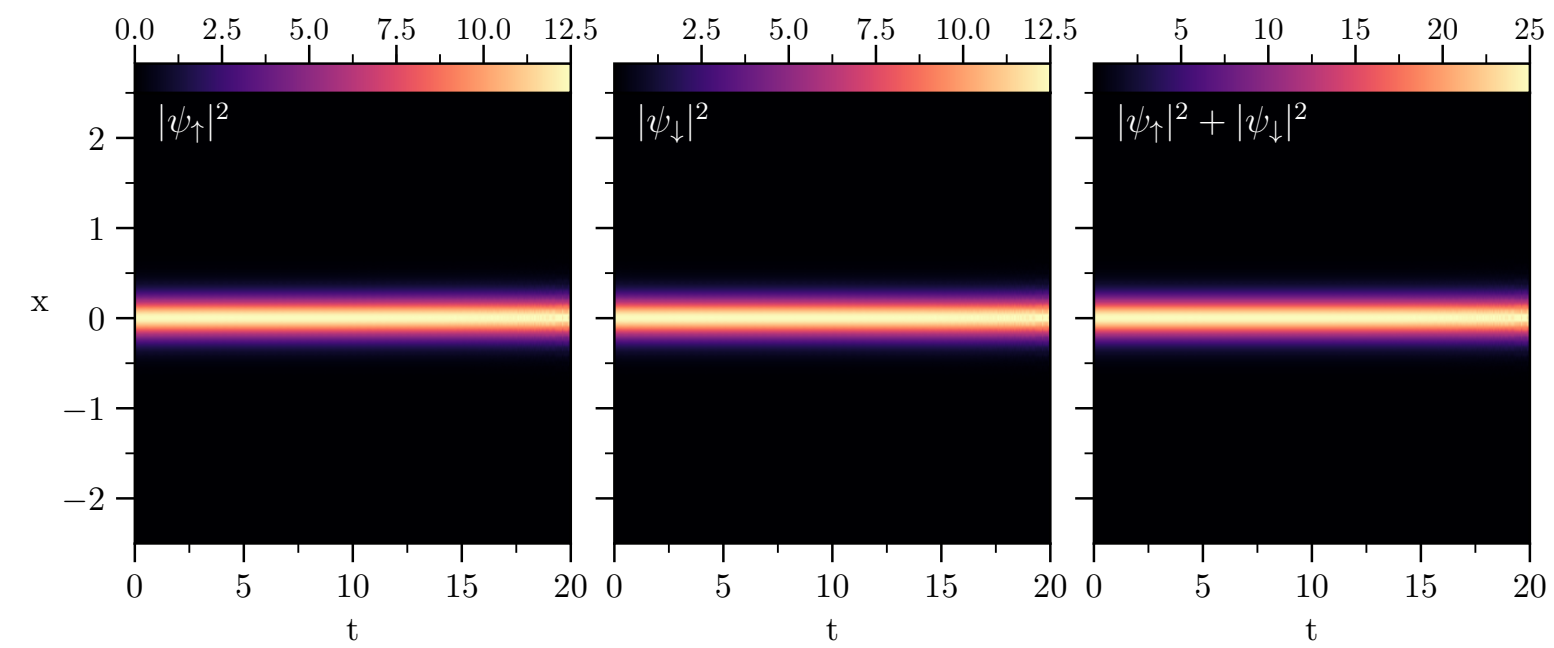

Figura 4.17: Evolução temporal das (2.99) e (2.100) para $\Omega=0.5, k_{L}=0.5 \sqrt{\Omega}, \delta=0$, usando na forma inicial (4.16), estados balanceados $\theta_{o}=\pi / 4, k_{o+}=0, k_{-}=k_{L}, \mathcal{N}=10, \eta=\mathcal{N} / 2 \mathrm{e}$ $\phi_{o-}=\pi / 2$.
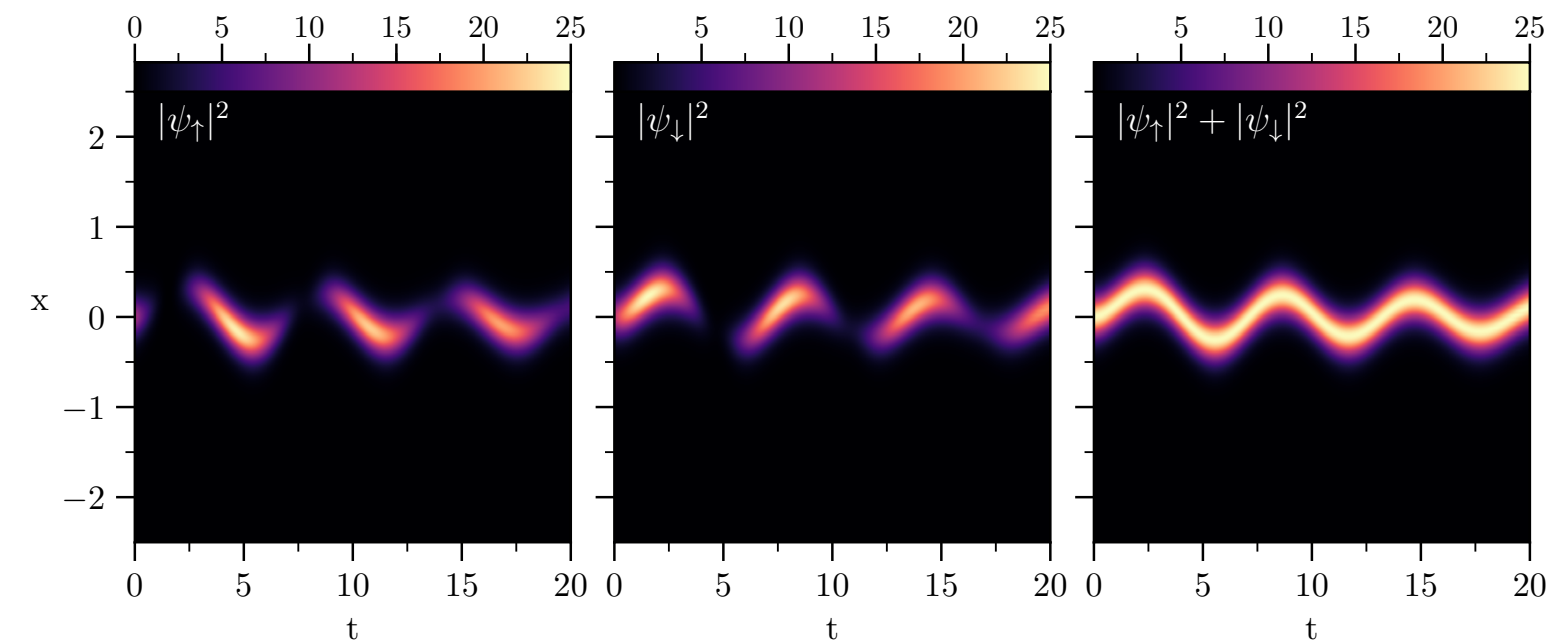

Figura 4.18: Evolução temporal das (2.99) e (2.100) para $\Omega=0.5, k_{L}=0.5 \sqrt{\Omega}, \delta=0$, usando na forma inicial (4.16), estados balanceados $\theta_{o}=\pi / 4, k_{o+}=0, k_{-}=k_{L}, \mathcal{N}=10$, $\eta=\mathcal{N} / 2$ e $\phi_{o_{-}}=\pi / 4$. Em contraste com a figura acima, a fase relativa aqui escolhida não anula as equações obtidas pelo variacional (4.18)-(4.22) e apresenta oscilações tanto no balanço do número de átomos em cada estado como do centro de massa.

A figura 4.17 mostra a ocasião descrita acima, onde a solução permanece estacionária. Caso se quebre a condição da fase relativa, a forma inicial começa oscilar no espaço seu centro de 


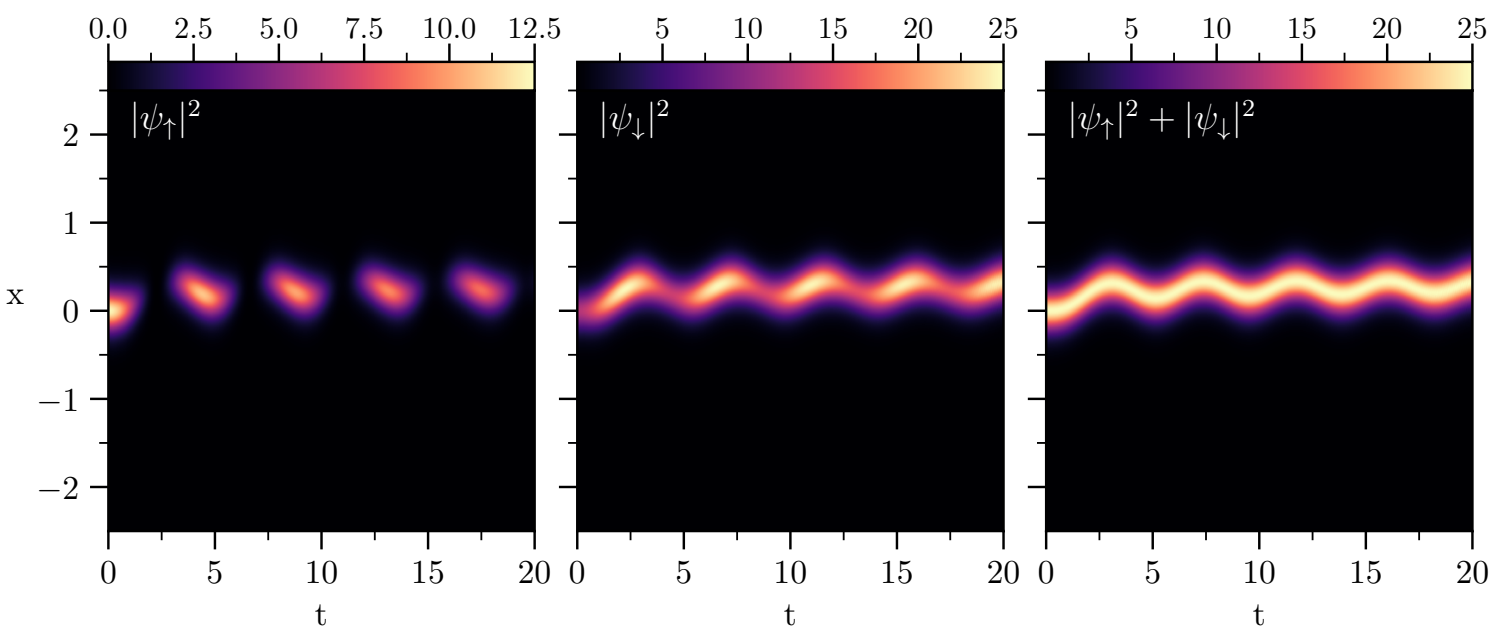

(a) $\delta=0.5$
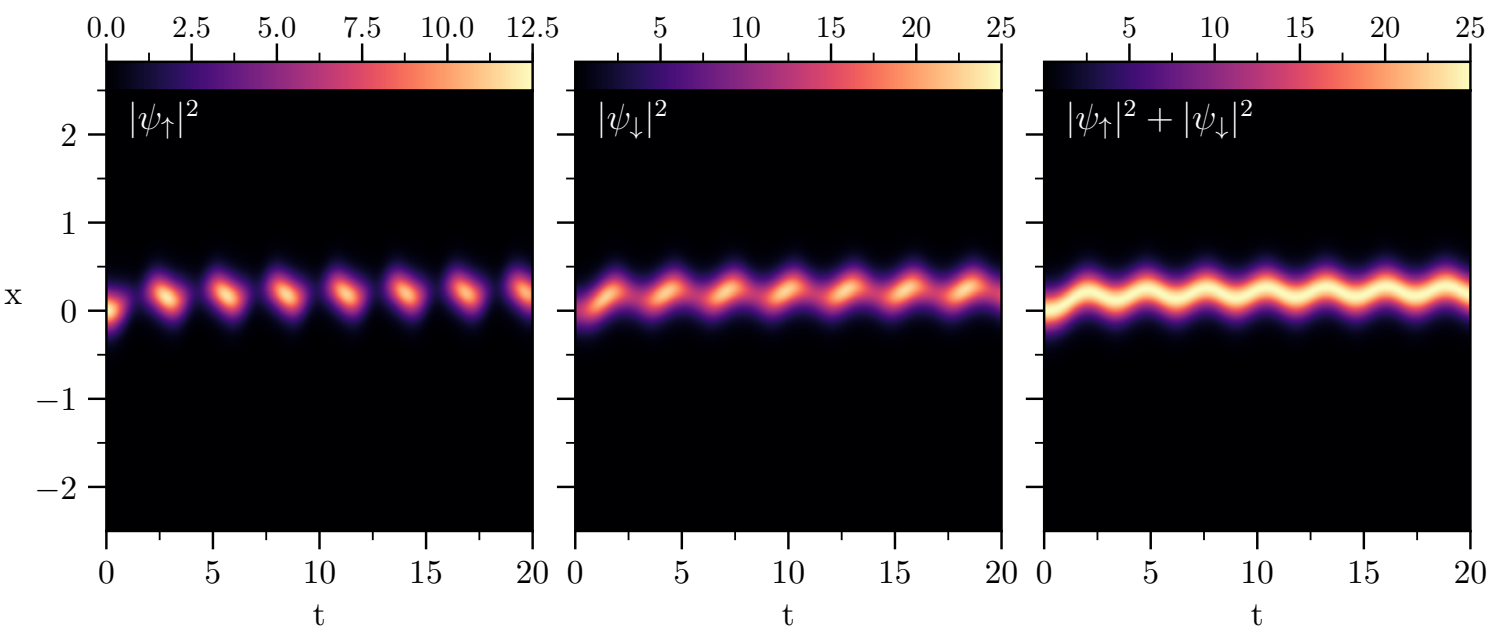

(b) $\delta=1.0$

Figura 4.19: Com as mesmas condições iniciais descritas na fig. 4.18, mas agora com $\delta$ não nulo observa-se o aumento da frequência de oscilação junto ao aumento de $\delta$, bem como a preferência por um dos subestados hiperfinos, que domina em número de átomos, revelado pela escala de cores no topo.

massa e o número de átomo em cado estado de spin, como pode ser visto na fig. 4.18.

Torna-se então de volta ao problema de analisar o efeito de $\delta$ sobre a dinâmica, que reside apenas na equação para evolução da fase relativa $\phi_{-}$. É fácil notar que

$$
\phi_{-}-\phi_{o_{-}}=-\delta t-\int_{0}^{t} \widetilde{\Omega} \cot (2 \theta) \cos \left(2 \phi_{-}+2 k_{L}\langle x\rangle\right)
$$

isto é, $\delta$ introduz uma frequência de oscilação, que influência na evolução de todos outros parâmetros.

De fato esta frequência de oscilação introduzida por $\delta$ é verificada na fig. 4.19, juntamente 

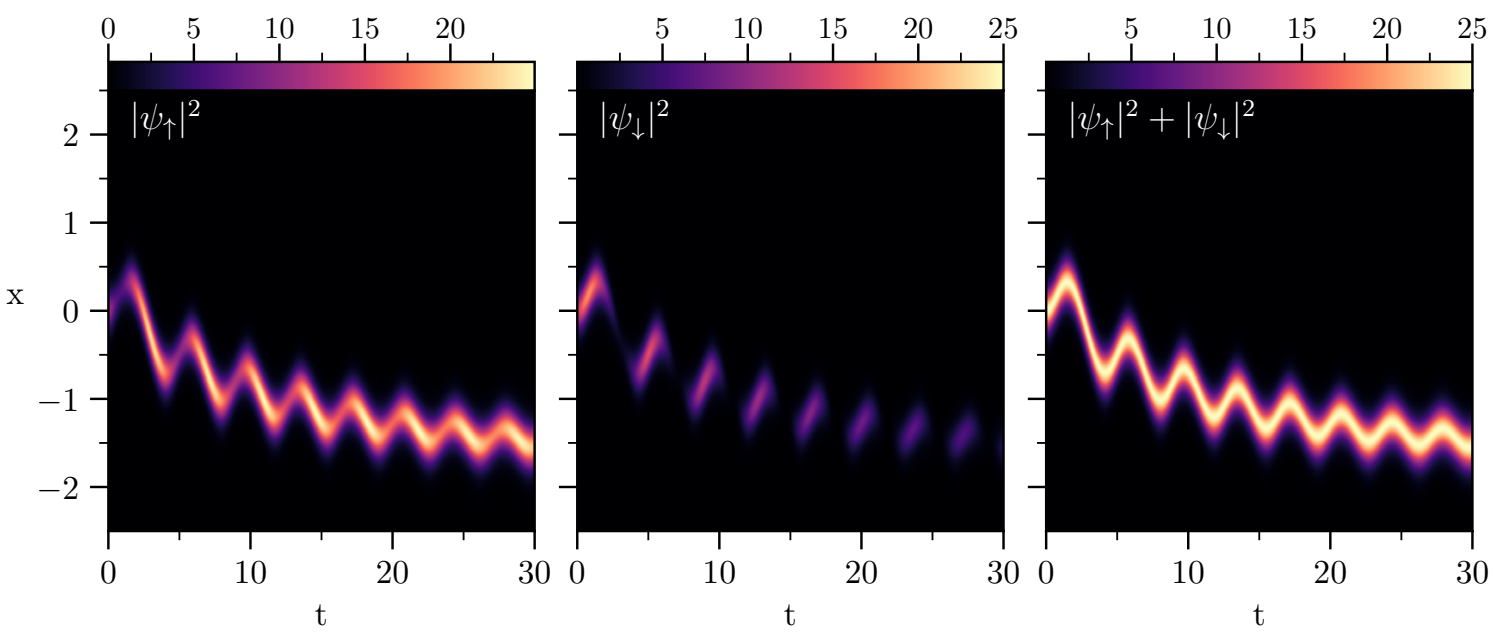

(a) $k_{o+}=0$
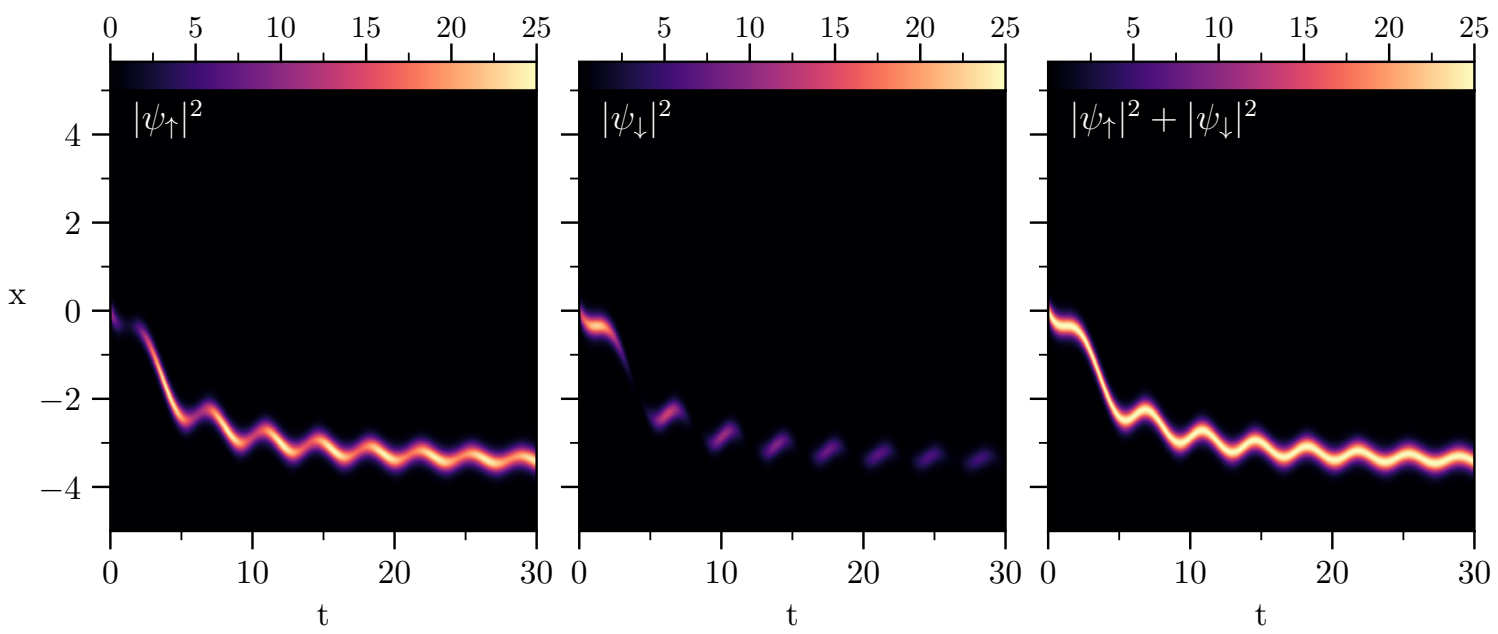

(b) $k_{o+}=-k_{L}$

Figura 4.20: Ainda com $\Omega=0.5$, mas agora com $k_{L}=0.8 \mathrm{e}, \delta=-0.64$. As condições iniciais, são as mesmas das usadas para gerar a fig. 4.18, com exceção da figura (b) a qual não mais se tem $k_{o+}=0$, valendo ainda ressaltar que $k_{-}$muda, uma vez mudado $k_{L}$. É notável que não se tem somente a oscilação puramente periódica mas um deslocamento mais efetivo que se acentua com o crescimento do valor de $k_{o+}$.

com a mudança deste parâmetro. Nota-se ainda um deslocamento inicial que implica $\langle x\rangle>0$ após completar o primeiro período.

Numa segunda análise, baseou-se numa escolha de parâmetros com $k_{L}^{2}>\Omega$ onde já se foi extensivamente discutido proporcionar mínimos em regiões de momento não nulo. Contudo, aqui não está sendo discutido um estado estacionário, ainda que, seja interessante se questionar desta mudança no contexto da dinâmica.

Na fig. 4.20 pode-se notar primeiro que mesmo mantendo $k_{o+}=0$ a mudança do parâmetro para $k_{L}^{2}>\Omega$ de fato afeta a dinâmica que agora não se restringe mais a apenas oscilação do 
centro de massa. Mudar $k_{o+}$ acentua tal efeito. Junto a esta mudança no movimento, nota-se uma parcela cada vez mais escassa de $\mathcal{N}_{\downarrow}$ durante a evolução, devido agora a $\delta<0$. 


\section{Capítulo 5}

\section{Conclusões e Perspectivas}

Neste estudo, foi mostrado um detalhamento do sistema físico para o acoplamento da dinâmica orbital e graus de liberdade internos, no caso dois subníveis do estado hiperfino $f=1$ e discutido amplamente como pode-se mudar cada parâmetro bem como suas limitações. A abundância de detalhes leva a uma interpretação completa e elucida futuros estudos que podem ser feitos.

Os resultados, de uma maneira geral, mostraram uma sistemática para obtenção de soluções estacionárias. Primeiramente estuda-se a diagonalização da hamiltoniana sem interação e, a partir de seu espectro faz-se uma proposição analítica(Ansatz) razoável, a partir do qual, podese recorrer a métodos numéricos para obtenção de soluções exatas, com a escolha da norma ou potencial químico fixo. Com isto possibilita-se a construção de diagramas com observáveis destes estados, como momento e desbalanço entre espécies que aqui foi extensivamente tratado, para diferentes parâmetros da equação.

Entende-se que reproduções tem sua importância em mostrar a credibilidade do que foi desenvolvido. Foi dada portanto a devida atenção em manter compatibilidade com outros estudos, reproduzindo-se por exemplo as regiões citadas em [13] e, levantando-se uma curva análoga a figura 3(a) de [17], que em particular é um trabalho experimental, mostrada aqui na figura 4.16.

Com a sistemática acima citada, mostrou-se em generalidade, a caracterização das fases numa ampla varredura de todos parâmetros da equação para interações atrativas, o que só foi estudado em um sistema periódico em [23] e discutido de forma sucinta em [22], mas não com a completeza aqui tratada.

Do que foi mostrado nas figuras da seção 4.3, observou-se que um sistema atrativo suaviza a transição da fase que se encontra em onda plana ou modulada, para a fase de momento zero, cujo o conceito passou a significar, interpretando as distribuições nos espaços de posição e momento, 
que $\langle|k|\rangle<\pi /(2 \sigma)$, onde $\sigma$ caracteriza a largura destas distribuições. Diferentemente, quando se estuda o sistema sem interação, este entra, segundo a relação $k_{L}^{2}=\Omega$ para $\delta=0$, no regime de um único mínimo exatamente a $k=0$.

A conclusão em torno do parâmetro $\delta$ é que este suprime a região da fase modulada, a qual dá lugar à fase de onda plana, evidenciado pelo cálculo variacional segundo as imagens com valores da correlação definida na equação (4.15), que indica uma sobreposição entre estados $\pm k$ onde centra-se a distribuição no espaço de momentos.

Com os observáveis mostrados fixando adicionalmente $k_{L}=7.5$, estes caracterizam pontos críticos onde não são diferenciáveis com relação a $\Omega$ se $\delta>0$, diferentemente para $\delta=0$, onde não indicam nenhuma variação brusca, vide gráficos na fig. 4.9. Contudo este valor crítico não diz respeito a nada de especial com a energia do estado fundamental, pois esta não apresenta pontos onde não se possa definir sua derivada ou descontinuidade.

Genericamente, conclui-se que o fator $\delta$ induz a transições bruscas nos observáveis para um certo domínio do parâmetro $\Omega$ que vem a depender também de $k_{L}$, evidenciado na figura 4.16, onde quanto menor o valor de $\Omega$ frente a $k_{L}$, o condensado reponde mais facilmente a pequenas variações de $\delta$, com uma mudança no momento total e no desbalanço do número de átomos em cada nível interno.

Sobre a dinâmica verificou-se a possibilidade de movimento coletivo dos átomos sem dispersão nem alteração em largura ou comprimento da forma inicial, conhecida como sóliton. Com um respaldo de cálculo variacional, mesmo mediante a diferentes condições, mostrouse que neste sistema tal solução não se propaga linearmente, ao invés, exibe oscilações de populações em cada nível interno e do centro de massa.

Contudo deve-se enfatizar, que estas oscilações podem ainda se sobrepor a um deslocamento efetivo ao redor do ponto de referência sobre o qual oscila, para certas condições iniciais e parâmetros da equação.

\section{Perspectivas de futuros estudos}

Os resultados aqui obtidos, sumarizam as diversas características do estado fundamental do sistema, para um sistema atrativo e, também da dinâmica dos átomos autoconfinados como soluções do tipo sóliton. Um prosseguimento destes estudos pode ser feito verificando-se:

1. Espectro de excitação do estado fundamental; 
2. Análise mais completa das possíveis transições de fase, acerca de descontinuidade dos observáveis;

3. Investigação da variação da força de interação e do número de átomos no sistema, eventualmente estudando junto a estabilidade;

4. Na dinâmica, estudo de variações temporais dos próprios parâmetros da equação, frisando obter batimentos ou mesmo ressonância;

5. Diagrama de fases para um sistema repulsivo com condições periódicas e, eventualmente conexão com uma geometria circular como estudado em [15]

Fora do contexto unidimensional, levanta-se outros pontos de interesse, sendo alguns já rotineiros na pesquisade condensados no entando pouco explorado para um sistema com acoplamento spin-orbital, como:

1. Vórtices e critérios de estabilidade;

2. Acoplamento entre spin e momento em mais de uma dimensão, como descrito na introdução de [17] dito ter apenas a contribuição Rashba;

3. Acoplamento entre todos subníveis do estado hiperfino $f=1$ como mencionado e trabalhado em $[18,44,28]$. 


\section{Apêndice A}

\section{Cálculo Variacional para Dinâmica de}

\section{Sólitons}

Baseado (4.16), podemos inseri-lo na Lagrangiana (2.63) e efetuar as integrais para obtê-la então em função dos parâmetros, com a modificação para as equações unidimensionais, ou seja, o funcional de energia dado portanto por (2.102), que por completeza ficam

$$
\begin{array}{r}
\frac{L(t)}{N}=\frac{1}{\mathcal{N}} \sum_{\sigma} \int_{-\infty}^{\infty} \mathrm{d} x \frac{i \hbar}{2}\left[\psi_{\sigma}^{*}(x, t) \frac{\partial \psi_{\sigma}}{\partial t}-\psi_{\sigma}(x, t) \frac{\partial \psi_{\sigma}^{*}}{\partial t}\right]-\frac{\mathcal{E}}{N}, \\
\frac{\mathcal{E}}{N}=\frac{1}{\mathcal{N}} \int_{-\infty}^{\infty} \mathrm{d} x\left\{\Psi^{\dagger}\left[-\frac{\partial^{2}}{\partial x^{2}}+i k_{L} \hat{\sigma}_{z} \frac{\partial}{\partial x}+\delta \sigma_{z}+\Omega \sigma_{x}\right] \Psi+\right. \\
\left.\frac{1}{2}\left( \pm\left|\psi_{\uparrow}\right|^{4}+\gamma\left|\psi_{\downarrow}\right|^{4}\right)+\beta\left|\psi_{\uparrow}\right|^{2}\left|\psi_{\downarrow}\right|^{2}\right\} .
\end{array}
$$

Onde por convenção foi tudo reescalonado pelo número de átomos, já que as equações de movimentos não devem mudar por esta constante. O conjunto de integrais de interesse são

$$
\begin{aligned}
& -\frac{1}{2} \int_{-\infty}^{\infty} \mathrm{d} x \Psi^{\dagger} \frac{\partial}{\partial x^{2}} \Psi=\frac{\mathcal{N}}{6}\left(3 \sin ^{2}(\theta) k_{\uparrow}^{2}+3 \cos ^{2}(\theta) k_{\downarrow}^{2}+\eta^{2}\right), \\
& i k_{L} \int_{-\infty}^{\infty} \mathrm{d} x \Psi^{\dagger} \frac{\partial}{\partial x} \hat{\sigma}_{z} \Psi=-k_{L} \mathcal{N}\left(\sin ^{2}(\theta) k_{\uparrow}-\cos ^{2}(\theta) k_{\downarrow}\right) \\
& \delta \int_{-\infty}^{\infty} \mathrm{d} x \Psi^{\dagger} \hat{\sigma}_{z} \Psi=\delta \mathcal{N}\left(1-2 \cos ^{2}(\theta)\right)=-\delta \mathcal{N} \cos (2 \theta), \\
& \Omega \int_{-\infty}^{\infty} \mathrm{d} x \Psi^{\dagger} \hat{\sigma}_{x} \Psi=\Omega \mathcal{N} \sin (\theta) \cos (\theta)\left[\frac{2 \pi k_{-}}{\eta \operatorname{sech}\left(\frac{\pi k_{-}}{\eta}\right)}\right] \cos \left(2 \phi_{-}-\frac{2 \xi k_{-}}{\eta}\right)
\end{aligned}
$$




$$
\begin{aligned}
& \frac{1}{2} \int_{-\infty}^{\infty} \mathrm{d} x\left(\left|\psi_{\uparrow}\right|^{4}+\left|\psi_{\downarrow}\right|^{4}\right)=\frac{\mathcal{N}^{2} \eta}{6}\left(\sin ^{4}(\theta)+\cos ^{4}(\theta)\right) \\
& \beta \int_{-\infty}^{\infty} \mathrm{d} x\left|\psi_{\uparrow}\right|^{2}\left|\psi_{\downarrow}\right|^{2}=\frac{\beta \mathcal{N}^{2} \eta}{3} \sin ^{2}(\theta) \cos ^{2}(\theta)
\end{aligned}
$$

$\operatorname{com} \phi_{ \pm} \doteq\left(\phi_{\uparrow} \pm \phi_{\downarrow}\right) / 2$ e $k_{ \pm} \doteq\left(k_{\uparrow} \pm k_{\downarrow}\right) / 2$. Para parte temporal deve-se notar que as derivadas podem ser expressas mediante regra da cadeia por

$$
\frac{\partial \psi_{\sigma}}{\partial t}=\sum_{A \in D} \frac{\partial \psi_{\sigma}}{\partial A} \dot{A}, \quad D=\left\{\eta, \xi, \theta, k_{\uparrow \downarrow}, \phi_{\uparrow \downarrow}\right\}
$$

As integrais de interesse resultam em

$$
\begin{aligned}
& \sum_{\sigma} \int_{-\infty}^{\infty} \mathrm{d} x \psi_{\sigma}^{*} \frac{\partial \psi_{\sigma}}{\partial \eta}=0 \\
& \sum_{\sigma} \int_{-\infty}^{\infty} \mathrm{d} x \psi_{\sigma}^{*} \frac{\partial \psi_{\sigma}}{\partial \theta}=0 \\
& \sum_{\sigma} \int_{-\infty}^{\infty} \mathrm{d} x \psi_{\sigma}^{*} \frac{\partial \psi_{\sigma}}{\partial k_{\sigma}} \dot{k}_{\sigma}=-i \frac{\mathcal{N} \xi}{\eta}\left(\dot{k}_{\uparrow} \sin ^{2}(\theta)+\dot{k}_{\downarrow} \cos ^{2}(\theta)\right) \\
& \sum_{\sigma} \int_{-\infty}^{\infty} \mathrm{d} x \psi_{\sigma}^{*} \frac{\partial \psi_{\sigma}}{\partial \phi_{\sigma}} \dot{\phi}_{\sigma}=i \mathcal{N}\left(\dot{\phi}_{\uparrow} \sin ^{2}(\theta)+\dot{\phi}_{\downarrow} \cos ^{2}(\theta)\right) .
\end{aligned}
$$

Escreve-se então a Lagrangiana, cancelando fatores de $\mathcal{N}$ e manipulando algumas das funções trigonométricas e transformação de variáveis, escreve-se

$$
\begin{aligned}
L(t)= & -\dot{\phi}_{+}+\dot{\phi}_{-} \cos (2 \theta)+\frac{\xi}{\eta}\left[\dot{k}_{+}-\dot{k}_{-} \cos (2 \theta)\right]-\frac{\eta^{2}}{6} \\
& -\frac{1}{2}\left[k_{+}^{2}+k_{-}^{2}-2 k_{+} k_{-} \cos (2 \theta)\right]+k_{L}\left[k_{-}-k_{+} \cos (2 \theta)\right] \\
& +\delta \cos (2 \theta)+\frac{\mathcal{N} \eta}{3}\left[\frac{1}{2}-(1-|\beta|) \frac{\sin ^{2}(2 \theta)}{4}\right] \\
& -\Omega \frac{\pi k_{-}}{\eta \operatorname{sech}\left(\pi k_{-} / \eta\right)} \sin (2 \theta) \cos \left(2 \phi_{-}-\frac{2 \xi k_{-}}{\eta}\right) .
\end{aligned}
$$

Segue-se então aplicando as equações de Euler-Lagrange para as funções temporais $A \in$ $\left\{\eta, \xi, \theta, k_{\uparrow \downarrow}, \phi_{\uparrow \downarrow}\right\}$

$$
\frac{\partial L}{\partial A}=\frac{\mathrm{d}}{\mathrm{d} t} \frac{\partial L}{\partial \dot{A}}
$$

Depois de trabalhar, substituindo uma equação em outra, com uma série de arranjos algébricos 
convenientes se chega a:

$$
\begin{gathered}
\langle\dot{x}\rangle=k_{+}-\left(k_{-}-k_{L}\right) \cos (2 \theta), \\
\dot{\theta}=-\frac{\Omega z}{\sinh (z)} \sin \left(2 \phi_{-}+2 k_{-}\langle x\rangle\right), \\
\dot{k}_{+}=\dot{k}_{-} \cos (2 \theta)+2 k_{-} \frac{\Omega z}{\sinh (z)} \sin (2 \theta) \sin \left(2 \phi_{-}+2 k_{-}\langle x\rangle\right), \\
\dot{\phi}_{-}=-\langle x\rangle \dot{k}_{-}-k_{+}\left(k_{-}-k_{L}\right)-\delta-\frac{\mathcal{N} \eta}{6} \cos (2 \theta)(1-|\beta|)- \\
\frac{\sinh (z)}{\Omega} \cot (2 \theta) \cos \left(2 \phi_{-}+2 k_{-}\langle x\rangle\right), \\
\frac{\eta^{2}}{3}-\frac{\mathcal{N} \eta}{3}\left[\frac{1}{2}-(1-|\beta|) \frac{\sin ^{2}(2 \theta)}{4}\right]=-k_{-}\left(k_{-}-k_{L}\right) \sin ^{2}(2 \theta) \\
\sinh ^{2}(z) \\
\cos \left(2 \phi_{-}+2 k_{-}\langle x\rangle\right)[z \operatorname{coth}(z)-1] \sin (2 \theta),
\end{gathered}
$$

onde $z=\pi k_{-} / \eta$ e $\langle x\rangle=\int \mathrm{d} x \Psi^{\dagger} x \Psi / \mathcal{N}=-\xi / \eta$, esta última a coordenada do centro de massa. É útil aqui enfatizar os limites

$$
\lim _{z \rightarrow 0} \frac{z}{\sinh (z)}=1, \text { e } \lim _{z \rightarrow 0}[z \operatorname{coth}(z)-1]=0
$$

os quais estão relacionados a não variação do parâmetro $\eta$, significando que o soliton mantém sua largura e altura e, portanto somente se desloca e, para que isso seja válido a igualdade central de (A.20) deve então satisfazer $k_{-}=0$ ou $k_{-}=k_{L}, \forall t$.

Nas condições citadas no parágrafo acima tem-se então

$$
\eta=\mathcal{N}\left[\frac{1}{2}-(1-|\beta|) \frac{\sin ^{2}(2 \theta)}{4}\right]
$$

onde naturalmente foi considerado $\eta \neq 0$. Nos estudos deste trabalho foi considerado interações simétricas frente aos estados de spin e portanto $\beta=-1.0$, conduzindo a $\eta=\mathcal{N} / 2$. Nota-se então que para os limites em (A.21) sejam aproximadamente satisfeitos para $k_{-}=k_{L}$ precisa-se que

$$
\mathcal{N} \gg 2 \pi k_{L}
$$


Tal condição se traduz no atual sistema de unidades a exigir que as interações sejam fortes o suficiente para o "soliton" de fato significar a descrição da seção 2.3.3 e não se dispersar.

Um outro regime que possa interessar, é a escolha de $k_{L}=0$, onde o movimento orbital não mais depende do spin. Nesta ocasião as transformações unitárias envolvidas na derivação da hamiltoniana nem sequer seriam necessárias e, o Ansatz em (4.16) poderia ser considerado $\operatorname{com} k_{\uparrow, \downarrow}=0$. Sendo então neste caso $z=0$ e podemos usar os valores dos limites em (A.21). Eliminando ainda todas as equações que se anulam de (A.16) a (A.20), termina-se com (A.22),

$$
\begin{aligned}
\dot{\theta} & =-\Omega \sin \left(2 \phi_{-}\right) \\
\dot{\phi}_{-} & =-\delta-\frac{\mathcal{N} \eta}{6} \cos (2 \theta)(1-|\beta|)-\Omega \cot (2 \theta) \cos \left(2 \phi_{-}\right)
\end{aligned}
$$

Com a definição de variável $y=-\cos (2 \theta) \operatorname{com} \theta \in[0, \pi)$ suficiente para varrer todo o período do argumento da função cosseno, temos $\sin (2 \theta)=\sqrt{1-y^{2}}$ e multiplicando por este fator a (A.24), encontra-se, após identificar uma regra da cadeia que

$$
\begin{aligned}
\dot{y} & =-2 \Omega \sqrt{1-y^{2}} \sin \left(2 \phi_{-}\right) \\
\dot{\phi}_{-} & =-\delta-\frac{\mathcal{N} \eta}{6}(1-|\beta|) y+\frac{\Omega y}{\sqrt{1-y^{2}}} \cos \left(2 \phi_{-}\right)
\end{aligned}
$$

Pode-se então traçar semelhança deste problema com outro sistema físico descrito em [43], quando se olha para as equações $2(\mathrm{a}, \mathrm{b})$ deste artigo. Por assim dizer $J=-\Omega, U$ a força de interação está incluso na normalização $\mathcal{N}, E_{L}^{0}-E_{R}^{0}=\delta$ e renomeando $z \equiv y$, pode-se chegar a equações quase idênticas, no entanto no referido trabalho é inserido um decaimento exponencial, de cunho fenomenológico e a forma inicial lá proposta não tem nem o formato ditado pela secante hiperbólica nem mesmo interação entre populações, i.e, $\beta=0$.

Ao contrário a interação entre populações é bem próxima de 1 e portanto teríamos mais interesse aqui para $|\beta|=1$. 


\section{Apêndice B}

\section{Cálculo Variacional para o Estado}

\section{Fundamental}

Como no apêndice anterior, o problema se baseia numa lista de integrais relevantes, para computar em (2.102) utilizando (4.13).

$$
\begin{aligned}
& -\frac{1}{2} \int_{-\infty}^{\infty} \mathrm{d} x \Psi^{\dagger} \frac{\partial}{\partial x^{2}} \Psi=\frac{k^{2}}{2}\left(\left|C_{+}\right|^{2}+\left|C_{-}\right|^{2}\right)+\frac{\mathcal{N}}{8 \sigma^{2}} \\
& i k_{L} \int_{-\infty}^{\infty} \mathrm{d} x \Psi^{\dagger} \frac{\partial}{\partial x} \hat{\sigma}_{z} \Psi=-k k_{L}\left(\left|C_{+}\right|^{2}+\left|C_{-}\right|^{2}\right) \cos (2 \theta) \\
& \quad \delta \int_{-\infty}^{\infty} \mathrm{d} x \Psi^{\dagger} \hat{\sigma}_{z} \Psi=-\delta\left(\left|C_{+}\right|^{2}-\left|C_{-}\right|^{2}\right) \cos (2 \theta) \\
& \Omega \int_{-\infty}^{\infty} \mathrm{d} x \Psi^{\dagger} \hat{\sigma}_{x} \Psi=-\Omega\left[\left(\left|C_{+}\right|^{2}+\left|C_{-}\right|^{2}\right) \sin (2 \theta)+\left(C_{+} C_{-}^{*}+C_{+}^{*} C_{-}\right) e^{\left.-2 k^{2} \sigma^{2}\right]}\right. \\
& -\int_{-\infty}^{\infty} \mathrm{d} x\left[\left(\left|\psi_{\downarrow}\right|^{4}+\left|\psi_{\uparrow}\right|^{4}\right)+|\beta|\left|\psi_{\uparrow}\right|^{2}\left|\psi_{\downarrow}\right|^{2}\right]=-\frac{1+|\beta|}{4 \sqrt{\pi} \sigma} \sin (2 \theta) \times \\
& \left.\quad\left(\left|C_{+}\right|^{2}+\left|C_{-}\right|^{2}\right)\left(C_{+} C_{-}^{*}+C_{+}^{*} C_{-}\right) e^{-k^{2} \sigma^{2}}+\sin (2 \theta)\left(C_{+}^{2} C_{-}^{* 2}+C_{+}^{* 2} C_{-}^{2}\right) e^{-4 k^{2} \sigma^{2}}\right]+ \\
& \quad-\frac{\left|C_{+}\right|^{4}+\left|C_{-}\right|^{4}}{4 \sqrt{\pi} \sigma}\left[1-\frac{1-|\beta|}{2} \sin (2 \theta)\right]-\frac{\left|C_{+}\right|^{2}\left|C_{-}\right|^{2}}{2 \sqrt{\pi} \sigma}[\sin (2 \theta)+|\beta|]
\end{aligned}
$$

Somando todas as integrais acima temos a energia expressa em termos dos parâmetros variacionais, onde ainda, para que o problema possa ser tratado numericamente deve-se expressar os coeficientes $C_{+}=\left|C_{+}\right| e^{i \phi_{1}}$ e $C_{-}=\left|C_{-}\right| e^{i \phi_{2}}$, para que a minimização envolva apenas parâmetros reais. 


\section{Referências Bibliográficas}

[1] J. Wilks and D. S. Betts. An Introduction to Liquid Helium. Oxford University Press, 1989.

[2] M. H. Anderson, J. R. Ensher, M. R. Matthews, C. E. Wieman, and E. A. Cornell. Observation of bose-einstein condensation in a dilute atomic vapor. Science, 269(5221):198-201, 1995.

[3] S. N. Bose. Plancks gesetz und lichtquantenhypothese. Zeitschrift für Physik, 26:168-171, Jul 1924.

[4] A. Einstein. Quantentheorie des einatomigen idealen gases. Verlag der KöniglichPreussischen, pages 3-14, jan 1925.

[5] W. Greiner, L. Neise, and H. Stöcker. Thermodynamics and Statistical Mechanics. Springer, first edition, 1995.

[6] R. K. Pathria and P. D. Beale. Statistical Mechanics. Elsevier, third edition, 2011.

[7] Lev Pitaevskii and Sandro Stringari. Bose-Einstein Condensation. Oxford University Press, New York, 2003.

[8] C. J. Pethick and H. Smith. Bose-Einstein Condensation in Dilute Gases. Cambridge University Press, Cambridge, 2004.

[9] Oliver Penrose and Lars Onsager. Bose-einstein condensation and liquid helium. Phys. Rev., 104:576-584, Nov 1956.

[10] L. D. Landau and E. M. Lifshitz. Quantum Mechanics (Non-relativistic Theory). Pergamon, Oxford, third edition, 1977. 
[11] Yuki Kawaguchi and Masahito Ueda. Spinor bose-einstein condensates. Physics Reports, $520(5): 253-381,2012$.

[12] Tin-Lun Ho. Spinor bose condensates in optical traps. Phys. Rev. Lett., 81:742-745, Jul 1998.

[13] V. Achilleos, D. J. Frantzeskakis, P. G. Kevrekidis, and D. E. Pelinovsky. Matter-wave bright solitons in spin-orbit coupled bose-einstein condensates. Phys. Rev. Lett., 110:4145, Jun 2013.

[14] Lin Wen, Q. Sun, Yu Chen, Deng-Shan Wang, J. Hu, H. Chen, W.-M. Liu, G. Juzeliūnas, Boris A. Malomed, and An-Chun Ji. Motion of solitons in one-dimensional spin-orbitcoupled bose-einstein condensates. Phys. Rev. A, 94:061602, Dec 2016.

[15] O. Fialko, J. Brand, and U. Zülicke. Soliton magnetization dynamics in spin-orbit-coupled bose-einstein condensates. Phys. Rev. A, 85:051605, May 2012.

[16] Alexander L. Fetter. Vortex dynamics in spin-orbit-coupled bose-einstein condensates. Phys. Rev. A, 89:23-29, Feb 2014.

[17] Y.-J. Lin, K. Jimenez-Garcia, and I. B. Spielman. Spin-orbit-coupled bose-einstein condensates. Nature, 471:83-86, Mar 2011.

[18] Y.-J. Lin, R. L. Compton, K. Jimenez-Garcia, J. V. Porto, and I. B. Spielman. Synthetic magnetic fields for ultracold neutral atoms. Nature, 462:628-632, Dec 2009.

[19] M. A. Khamehchi, Khalid Hossain, M. E. Mossman, Yongping Zhang, Th. Busch, Michael McNeil Forbes, and P. Engels. Negative-mass hydrodynamics in a spinorbit coupled bose-einstein condensate. Phys. Rev. Lett., 118:155301, Apr 2017.

[20] R. A. Williams, L. J. LeBlanc, K. Jiménez-García, M. C. Beeler, A. R. Perry, W. D. Phillips, and I. B. Spielman. Synthetic partial waves in ultracold atomic collisions. Science, 335:314-317, Jan 2012.

[21] Zeng-Qiang Yu. Landau criterion for an anisotropic bose-einstein condensate. Phys. Rev. A, 95:033618, Mar 2017.

[22] Y. Zhang, M. E. Mossman, T. Busch, P. Engels, and C. Zhang. Properties of spin-orbitcoupled bose-einstein condensates. Frontiers of Physics, 11(3):118103, 2016. 
[23] Yun Li, Giovanni I. Martone, and Sandro Stringari. Spin-orbit-coupled Bose-Einstein Condensates, chapter 5, pages 201-250. World Scientific, 2015.

[24] Eugen Merzbacher. Quantum Mechanics. John Wiley \& Sons, third edition, 1998.

[25] Kurt Gottfried and Tung-Mow Yan. Quantum Mechanics: Fundamentals. Springer, second edition, 2003.

[26] Sadri Hassani. Mathematical Physics: A Modern Introduction to Its Foundations. Springer, second edition, 2012.

[27] Steven Weinberg. Lectures on Quantum Mechanics. Cambridge University Press, first edition, 2013.

[28] Y.-J. Lin, R. L. Compton, A. R. Perry, W. D. Phillips, J. V. Porto, and I. B. Spielman. Bose-einstein condensate in a uniform light-induced vector potential. Phys. Rev. Lett., 102:04-07, Mar 2009.

[29] N. Goldman, G. Juzeliūnas, P. Ohberg, and I. B. Spielman. Light-induced gauge fields for ultracold atoms. Reports on Progress in Physics, 77(12):126401, 2014.

[30] E. Brion, L. H. Pedersen, and K. Mølmer. Adiabatic elimination in a lambda system. Journal of Physics A: Mathematical and Theoretical, 40(5):1033, 2007.

[31] J. Crank and P. Nicolson. A practical method for numerical evaluation of solutions of partial differential equations of the heat-conduction type. Mathematical Proceedings of the Cambridge Philosophical Society, 43(1):50-67, 1947.

[32] Douglas Quinney. An Introduction to the Numerical Solution of Differential Equations. Research Studies Press LTD, England, 1987.

[33] K. W. Morton and David Mayers. Numerical Solution of Partial Differential Equations. Cambridge University Press, United Kingdom, 2005.

[34] Paul L. DeVries. A First Course in Computational Physics. John Wiley \& Sons, Ohio, 1994.

[35] J. Yang. Newton-conjugate-gradient methods for solitary wave computations. Journal of Computational Physics, 228:7007-7024, 2009. 
[36] William H. Press, Saul A. Teukolsky, William T. Vetterling, and Brian P. Flannery. Numerical Recipes in $C$ - The Art of Scientific Computing. Cambridge University Press, Cambridge, 1992.

[37] Jonathan R. Shewchuk. An introduction to the conjugate gradient method without the agonizing pain. Technical report, Pittsburgh, PA, USA, 1994.

[38] G. Meurant. Computer Solution of Large Linear Systems. Elsevier, 1999.

[39] Y. Saad. Iterative Methods for Sparse Linear Systems. SIAM, Philadelphia, 2003.

[40] Gerard L.G. Sleijpen and Diederik R. Fokkema. Bicgstab(1) for linear equations involving unsymmetric matrices with complex spectrum. Electronic Transactions on Numerical Analysis, 1:11-32, 1993.

[41] Claude Pommerell. Solution of Large Unsymmetric Systems of Linear Equations. PhD thesis, Swiss Federal Institute of Technology of Zurich, 1992.

[42] Martin H. Gutknecht. Variants of bicgstab for matrices with complex spectrum. SIAM Journal on Scientific Computing, 14:1020-1033, 1993.

[43] M. Abbarchi, A. Amo, V. G. Sala, D. D. Solnyshkov, H. Flayac, L. Ferrier, I. Sagnes, E. Galopin, A. Lemaître, G. Malpuech, and J. Bloch. Macroscopic quantum self-trapping and josephson oscillations of exciton polaritons. Nature Physics, 9:275-279, Apr 2013.

[44] I. B. Spielman. Raman processes and effective gauge potentials. Phys. Rev. A, 79:063613, Jun 2009. 